\title{
Zum Alltagsleben der Ada \\ Tepe Goldproduzenten \\ im 15. Jh. v. Chr. Das \\ Fundensemble aus Haus 7 \\ in funktionaler und \\ kontextueller Analyse
}

\author{
Barbara Horejs
}

\begin{abstract}
Zusammenfassung
Die hier vorgelegte Studie ist die erste Vorlage eines gesamten Fundensembles eines spätbronzezeitlichen Hauses vom Ada Tepe. Das Inventar aus Haus 7 erlaubt uns einen ersten Einblick in das Alltagsleben der Goldproduzenten im 15. Jh. v. Chr. Die Untersuchung der Gefäßformen und ihrer technologischen Waren aus Haus 7 zeigt ein homogenes Spektrum aus annähernd standardisierten Typen und Waren. Ihre funktionale und kontextuelle Auswertung veranschaulicht eine vor allem auf Lagerung und weniger auf Konsum beschränkte Nutzung. Die deutlich dominierenden Amphoren und engmundigen Töpfe dürften vor allem zur Speicherung von Lebensmitteln gedient haben. Das in geringerem Maße vorkommende Tafelgeschirr aus einem Set von Schalen, Schüsseln, Tassen bzw. Bechern sowie Kantharoi verweist möglicherweise auf Aktivitäten von Essen und Trinken direkt im Speicherbau, in dem auch verschiedenes häusliches Handwerk (Mahlen, Schneiden, Sägen) stattgefunden haben dürfte. Haus 7 kann aufgrund dieser Ergebnisse als separater Lagerund Arbeitsraum interpretiert werden, was auf eine organisierte Versorgung und Vorratshaltung der Goldproduzenten schließen lässt. Die ersten hier unternommenen regionalen wie überregionalen Vergleiche des Keramikinventars zeigen vor allem eine lokale Einbettung in den Kulturhorizont der Rhodopen mit erkennbaren Verbindungen sowohl in die zentral- bis nordbulgarischen Regionen als auch in die südlich benachbarte Nordägäis.
\end{abstract}

\section{Schlüsselbegriffe}

Ada Tepe, Spätbronzezeit, Siedlung, Keramik, Kontextanalyse, Alltagsleben.

Abstract - On the Everyday Life of the Ada Tepe Gold Producers in the $15^{\text {th }}$ Century BC. The Find Assemblage from House 7 in Functional and Contextual Analysis

This study represents the first publication of a complete find assemblage from a Late Bronze Age house at Ada Tepe. The inventory of House 7 offers a preliminary insight into the everyday life of the Ada Tepe gold-mining society in the $15^{\text {th }}$ century BC. Detailed study of the pottery shapes and fabrics shows that there was a homogeneous spectrum of nearly standardised types and wares. Their functional and contextual evaluation demonstrates that House 7 was used principally for storage and to a lesser degree for consumption. The shapes that clearly dominate the material, amphorae and narrow-mouthed jars, seem to have been used mainly for the storage of food and liquids. The less common table ware, belonging to a set of shallow bowls, deep bowls, cups or beakers and kantharoi, probably relates directly to the consumption of food and drink inside the storage building, in which various household activities are also attested, such as grinding, cutting and sawing. On the basis of these analyses, House 7 can be interpreted as a separate space for storage and craftwork which allows us to assume that the gold miners organised their supply and storage systems. This assessment of the pottery assemblage in a regional and supra-regional setting indicates a society that was principally embedded in the local culture of the Rhodope mountains, but has recognisable links to the regions of central and north Bulgaria and also, to the south, with the north Aegean.

\section{Keywords}

Ada Tepe, Late Bronze Age, settlement, pottery, contextual analysis, everyday-life.

\section{Einleitung}

Das in vielerlei Hinsicht bedeutende Goldbergwerk des Ada Tepe eröffnet der südosteuropäischen Bronzezeitforschung bisher verschlossene Einblicke in die Lebenswelt von Bergleuten des 2. Jts. v. Chr. Das einzigartige archäologische Ensemble des Ada Tepe beinhaltet neben den Abbaustollen, Halden und Arbeitsarealen des Bergwerks auch verschieden gestaltete Siedlungszonen. ${ }^{1}$ Es liegt nahe, hier

1 Zur Übersicht des Ada Tepe siehe Popov et al. 2017 in diesem Band sowie Popov, Jockenhövel 2011. - Popov, Jockenhövel, Groer 2011. - Popov et al. 2011. - Popov et al. 2014. - Popov, Nikov, JoCKENHÖVEL 2015. 
die Unterbringung der Bergleute und möglicherweise die mit ihnen verbundenen sozialen Gruppen (Kernfamilien, Verbände, Clans) zu vermuten. Diese Ausgangshypothese eines direkten Zusammenhangs zwischen dem Bergwerk und den Siedlungen auf dem Ada Tepe lässt sich durch einige Indizien erhärten. Eine große Serie von Radiokarbondaten weist auf gleichzeitige Aktivitäten beim Goldabbau sowie in den Siedlungsarealen mit jeweils unterschiedlichen Entsprechungen hin. Eine detaillierte Modellierung der gesamten Chronologie ist zwar noch in Vorbereitung, doch zeigen die vorläufigen Ergebnisse bereits vergleichbare $\mathrm{Da}$ ten des ältesten Goldabbaus mit dem Nordostquartier des Ada Tepe im 15. Jh. v. Chr. ${ }^{2}$ Ein weiterer Hinweis ist das Vorkommen von typischen Werkzeugen und Geräten innerhalb der Siedlung (Klopfsteine, Reibsteine u. ä), wie sie auch in der Bergwerkszone verwendet wurden. Schließlich ist eine parallele, aber zum Goldabbau völlig unabhängige Ansiedlung eher unwahrscheinlich. Es ist also sehr wahrscheinlich anzunehmen, in den Resten der Siedlungen auch Erkenntnisse über das Leben der Bergwerks-Gesellschaft zu finden.

Für unser Verständnis der primären Produzenten des Ada Tepe Goldes ist diese Hypothese essentiell, da auf sie aus der Bergwerkszone selbst - abgesehen von Indizien zu den Arbeitsprozessen - kaum Hinweise erhalten sind. Aus anderen Bergwerken steht uns hingegen eine Fülle an Informationen zum Alltagsleben zur Verfügung, wobei hier vor allem die konservierenden Salzbergwerke wie Hallstatt in den Alpen oder Chehrābād im Iran genannt werden können. ${ }^{3}$ Aus den anthropologischen Analysen zum Gräberfeld des Hallstätter Hochtals wurde geschlossen, dass Kinder, Jugendliche, Frauen und Männer in den Arbeitsprozess der Salzgewinnung eingebunden waren. ${ }^{4}$ Inwiefern wir auch auf dem Ada Tepe mit einer solchen Arbeitseinbindung verschiedener Bevölkerungsgruppen zu rechnen haben, muss bis zur Entdeckung der dazugehörigen Nekropole natürlich offen bleiben. Dennoch ist die Einbeziehung dieser Erkenntnisse aus Hallstatt interessant, wenn wir die dazugehörigen Siedlungsreste des Ada Tepe analysieren und interpretieren möchten. Schließlich liefern uns die Bebauung

\footnotetext{
2 Eine erste Übersicht zur Chronologie bei Popov et al. 2017 in diesem Band sowie Popov, Nikov im Druck. - Die Publikation der Chronologie-Modellierung wird derzeit von B. Weninger in Zusammenarbeit mit R. Jung und $\mathrm{H}$. Popov vorbereitet.

3 Stöllner, Oeggl 2015 mit weiterführender Literatur zu verschiedenen Bergwerken. - Kern et al. 2008. - Aali, STöLlner 2015. - Beispielhaft zum Aspekt der Bekleidungen und organischen Behältnisse im Bergwerk siehe Russ-Popa 2008 und Grömer 2010. 4 Pany 2008.
}

und ihre Funde wichtige Indikatoren zu einer ersten Interpretation des Alltagslebens der Goldproduzenten des Ada Tepe und erlauben uns Rückschlüsse auf ihre profanen Aktivitäten auf dem Berg.

Die vorliegende Studie ist ein Ergebnis einer laufenden Kooperation des Nationalen Archäologischen Instituts mit Museum (NAIM) der Bulgarischen Akademie der Wissenschaften mit dem Institut für Orientalische und Europäische Archäologie (OREA) von der Österreichischen Akademie der Wissenschaften, das im Rahmen eines vom Österreichischen Fonds zur Förderung der Wissenschaftlichen Forschung (FWF) finanzierten Projektes durchgeführt wird. Ziel ist die Untersuchung des von Hristo Popov und seinem Team ausgegrabenen Nordostquartiers auf dem Ada Tepe sowie der Rolle von Goldproduzenten und -konsumenten im spätbronzezeitlichen Südosteuropa. ${ }^{5}$

\subsection{Eliten und Alltag}

Über die Strukturen spätbronzezeitlicher Gesellschaften im heutigen Bulgarien stehen uns nur wenige Informationen zur Verfügung, die vor allem auf Untersuchungen von Gräberfeldern und Horten basieren. ${ }^{6}$ Die spätbronzezeitlichen Depots und Bestattungen in Bulgarien erlauben uns einen indirekten Einblick in komplexe und differenzierte gesellschaftliche Gruppen, aus denen nun auch immer deutlicher potentielle Eliten hervortreten. Dazu gehören beispielsweise die Bestattungen von Izvorovo und Ovčarci oder das erst jüngst entdeckte Depot von Svištov.? Ihnen gemeinsam ist eine besonders reiche Ausstattung nicht nur mit hochwertigem goldenen Schmuck, sondern auch vermutlichen Importen. Diese teilweise auch als standardisierte Statussymbole vorkommenden goldenen Halsringe (Torques) sowie Schläfen-, Locken- oder Ohrringe weisen auf eine gesellschaftliche Gruppe hin, ${ }^{8}$ die sich in den üblichen Siedlungen des 2. Jts. v. Chr. bisher kaum fassen ließ. Inwieweit die durch Waffen indirekt erschließbaren Kontakte zu ägäischen Kriegern auch in diesem elitären Kontext zu verorten sind, müssen zukünftige kontextuelle Studien erst untersuchen. Bisher weist ihr Vorkommen im 15. und 14. Jh. v. Chr. aber zumindest auf einen Personenkreis

\footnotetext{
5 Für weitere Information zu diesen Forschungen siehe auch $<$ http:// www.orea.oeaw.ac.at/forschung/mediterranean-economies/ bronze-age-gold-road-of-the-balkans-ada-tepe-mining/> (letzter Zugriff 16.5.2017).

6 Leshtakov 2008. - Athanassov 2011. - Athanassov, Krauss 2015.

7 Borislavov 2017. - Alexandrov 2017. - Alexandrov, ChrisTOVA, MARINOv 2017 (jeweils mit weiterführender Literatur). 8 Für einen Überblick siehe HAAg et al. 2017, 171-173.
} 


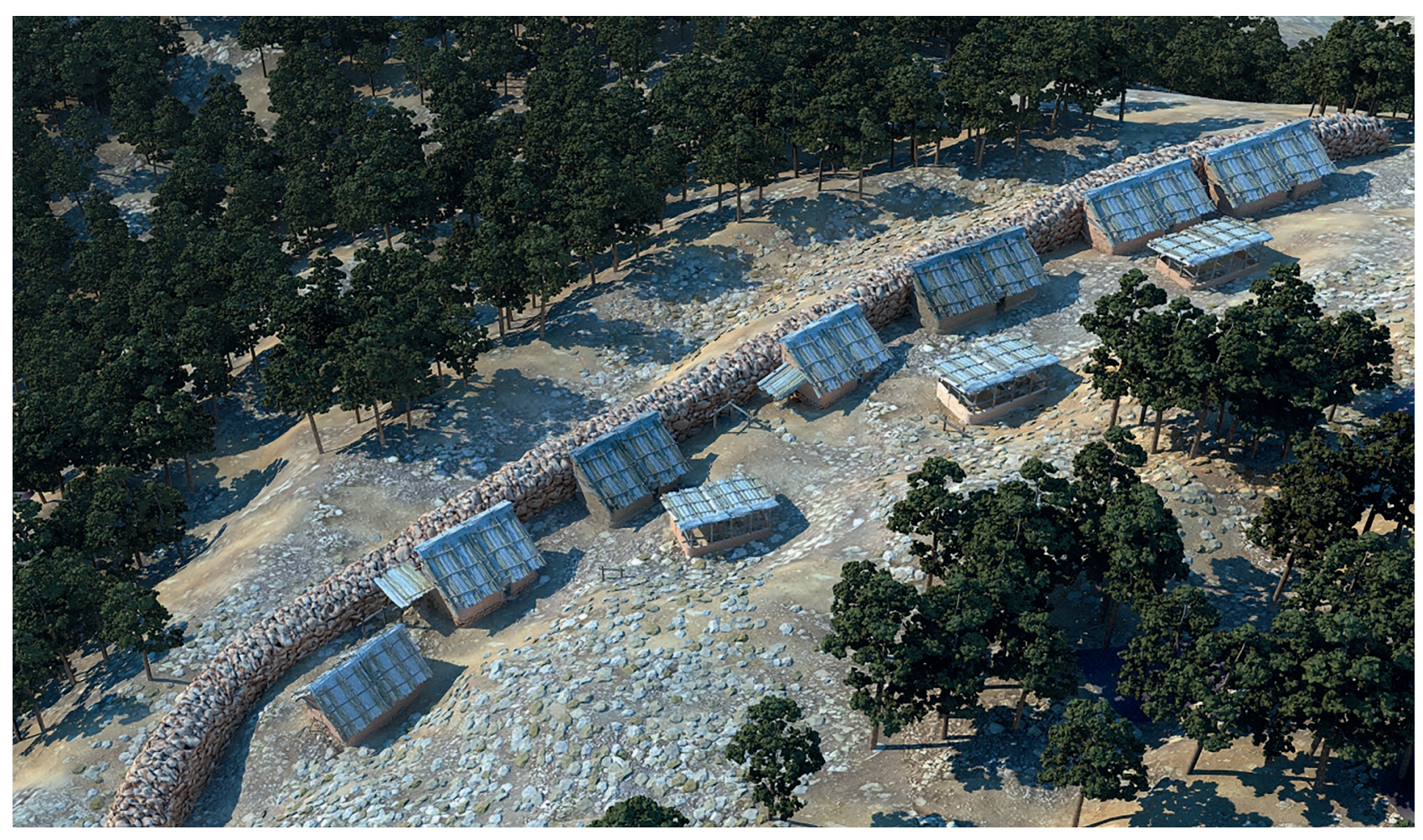

Abb. 1. Rekonstruktion des Nordostquartiers auf dem Ada Tepe. Die Eingänge der Häuser der hinteren Reihe befinden sich in dieser Darstellung an der Langseite. Sie könnten sich aber auch an der Schmalseite befunden haben, wie Popov et al. 2017 in diesem Band näher ausführen (Grafik: M. Klein, 7reasons media; OREA; NAIM 2017).

hin, der diese ägäischen Schwerter sowohl importierte als auch imitierte. ${ }^{9}$ Ein Indiz für eine überregional vernetzte Gesellschaftsgruppe im profanen Kontext ist wohl in der importierten mykenischen Drehscheibenkeramik zu sehen, wie Jung und KollegInnen in diesem Themenspecial für die Siedlungen von Koprivlen und Dragojna diskutieren. ${ }^{10}$ Mit den Siedlungsresten vom Ada Tepe eröffnet sich nun die Möglichkeit, die lokalen Aktivitäten abseits des Bergbaus zu analysieren, um einen möglichen „Alltagshorizont“ $\mathrm{zu}$ erschließen, der sich zumindest auf den ersten Blick sehr deutlich von den oben erwähnten Gesellschaftsgruppen der Goldschmuck- und Schwertträger unterscheiden dürfte.

Die hier diskutierten Überlegungen zum Alltagsleben auf dem Ada Tepe im 15.Jh.v. Chr. basieren auf funktionalen und kontextuellen Analysen des Fundensembles aus Haus 7 im Nordostquartier. Dieses Hausinventar wurde von der Autorin 2015 im Depot von Krumovgrad aufgenommen, um die Basis für eine lokal definierte Systematik des Projekts zu erstellen. Die hiermit auch erstmals vorgestellte Typologie von Waren und Formen bildet gleichsam den Auftakt weiterer Materialstudien, die auf dieses Grundgerüst und die

9 Athanassov, Krauss, Slavčev 2009. - Zur jüngsten Zusammenstellung von Schwertern und ihrer Interpretation siehe Jung 2017. 10 Jung et al. 2017 in diesem Band.
Primärdaten des Inventars zurückgreifen können, und ist deshalb entsprechend ausführlich dargelegt.

\section{Archäologische Rahmenbedingungen und Methoden}

\subsection{Haus $7 \mathrm{im}$ Nordostquartier}

Das Siedlungsareal im Nordosten des Ada Tepe besteht aus insgesamt elf gebauten Strukturen, die offenbar in Bezug aufeinander angelegt wurden (Abb. 1-2). ${ }^{11}$ Die unterschiedliche Konstruktionsweise und Form der Strukturen entlang der Umfassungsmauer einerseits und der in den Hang hineingesetzten Bauten andererseits lässt auf eine mögliche divergente Nutzung schließen. Die Hanglage von Haus 7 führte zu einer Überlagerung der archäologischen Strukturen mit erodierenden Schichten vom Berg sowie Schutt von spätbronzezeitlichen Abbauhalden, wie die Befundauswertung von H. Popov und seinen KollegInnen in diesem Band zeigt. Um die stratigrafischen Erkenntnisse eingangs nochmals kurz zusammenzufassen, handelt es sich bei Haus 7 um einen ovalen $6 \times 3,50 \mathrm{~m}$ großen Bau, der aus einem Lehm-Flechtwerk-Aufbau konstruiert wurde.

11 Siehe dazu ausführlich H. Popov et al. 2017 in diesem Band. 


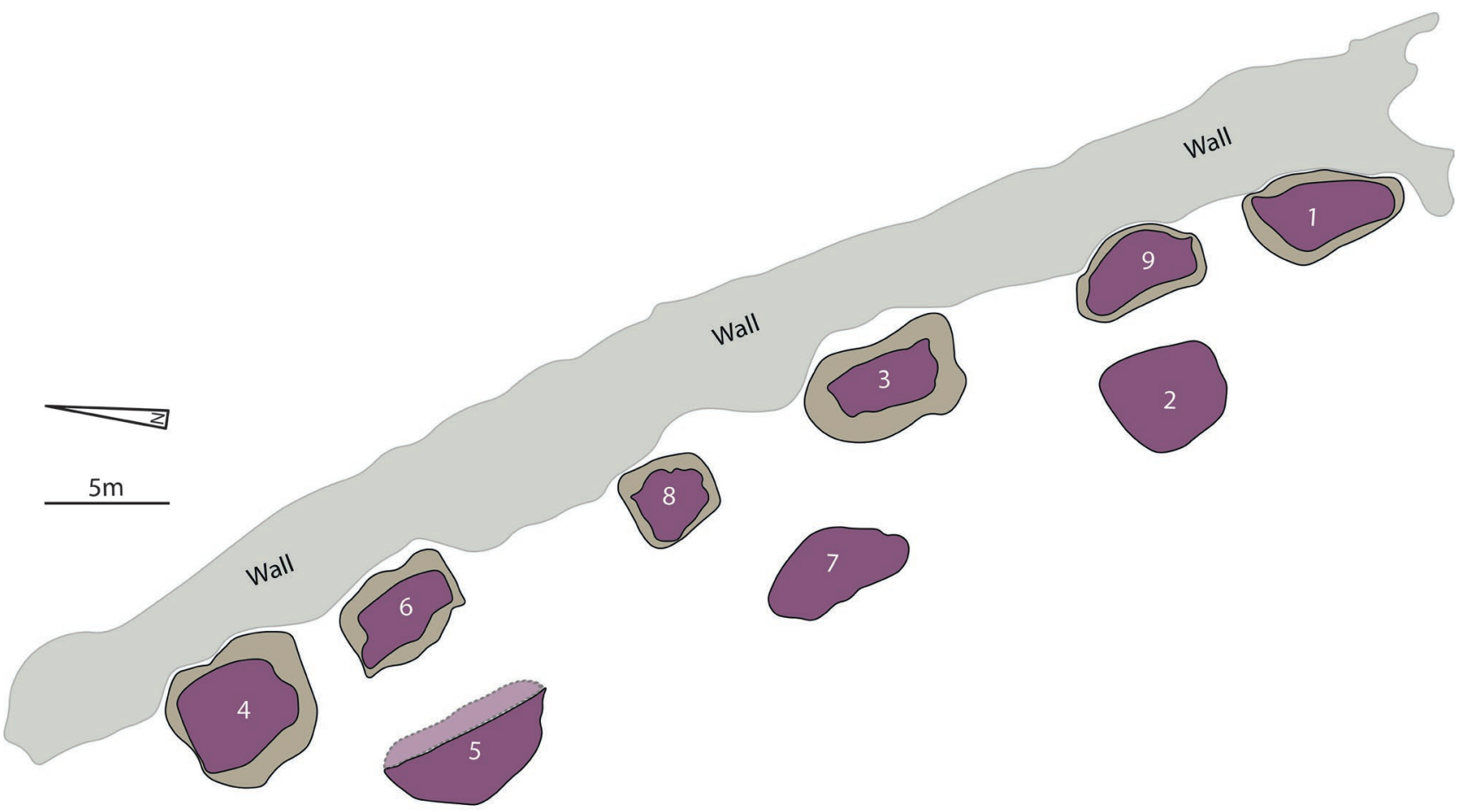

Abb. 2. Schematischer Plan der Bebauung im Nordostquartier des Ada Tepe. Befunde: violett, Mauerstrukturen: beige (Grafik: L. Burkhardt, OREA; mit freundlicher Genehmigung von H. Popov, NAIM).

In der Rekonstruktion schließen wir auf ein Pfosten-gestütztes Dach sowie möglicherweise nur zur Hälfte hochgezogene Wände, was aber hypothetisch bleibt, da vom Aufbau selbst nichts erhalten war. Die unterhalb der Erosions- und Versturzschichten freigelegten Befunde können als Kontexte zum Zeitpunkt der Hangablagerungen über dem Haus definiert werden. Die starke Fragmentierung der Gefäßkeramik und das weitgehende Fehlen von (rekonstruierbaren) Ganzgefäßen lassen auf eine mögliche Zeitspanne mit Erosions- und Verlagerungsprozessen zwischen letzter Nutzung und endgültiger Zerstörung schließen. Das Inventar ist daher zwar nicht ein vollständiges in situ gefundenes Ensemble, aber dennoch ein Kontext, der die gesamte Nutzung des Baus widerspiegelt (Abb. 2). Das Fehlen ausgebesserter bzw. erneuter Fußböden lässt auf eine überschaubare zeitliche Phase der Nutzung von Haus 7 im 15. Jh. v. Chr. schließen. Die Befundsituation weist nicht auf komplexe Deponierungsprozesse hin, sondern lässt uns ein einphasiges Gebäude rekonstruieren.

Die Auswertung des Inventars und seiner Fundsituation bekräftigt diese Annahme (siehe unten). Inwieweit dies im Kontext mit den anderen Bauten des Nordostquartiers zu beurteilen ist, werden erst die Modellierung der Gesamtchronologie des Berges sowie die Detailauswertung aller
Inventare zeigen können. Zwei Faktoren sollen hier abschließend betont werden, die Popov et al. in diesem Themenspecial herausgearbeitet haben: Die Häuser im Nordostquartier haben keine lange Nutzungsdauer, was sowohl die geringe Mächtigkeit der Kulturschichten, die Konstruktionsweise der Häuser als auch die Radiokarbondaten nahelegen. Zudem wurde das Areal durch Hangerosion und spätbronzezeitlichen Haldenschutt überlagert, was die Häuser noch innerhalb derselben Periode versiegelte. Damit sind die Ensembles aus den Bauten zwar keine per se „geschlossenen Kontexte", lassen sich aber auf eine kurze Nutzungsphase innerhalb des 15. Jhs. v. Chr. begrenzen. Inwieweit die Funde aber tatsächlich die Deponierung von darin verwendeten Gefäßen und Werkzeugen zu einem einzigen Zeitpunkt widerspiegeln oder aus mehreren Jahrzehnten stammen, muss offen bleiben. Spätere, nach der Auflassung dieser Siedlung eingebrachte Objekte, lassen sich sehr wahrscheinlich ausschließen, da die Architektur und dazugehörige Kulturschichten unmittelbar mit Erosion (chronologisch bevor der Haldenschutt alles überlagerte) überdeckt waren. Insofern können wir das Ensemble aus Haus 7 derzeit nur als Spektrum seiner gesamten Nutzungszeit verstehen, die von der Errichtung bis zur Auflassung dauerte, insgesamt aber kaum mehrere Jahrzehnte umfasst haben dürfte. 


\subsection{Methoden der Keramikanalyse}

Die Materialaufnahme des Nordostquartiers folgt den bereits andernorts angewendeten und bewährten Systematiken, unter anderem auch, um die Kompatibilität der Ergebnisse zu gewährleisten. ${ }^{12}$ Diesem System folgend wurden alle Keramikfragmente nach Kontexten ausgelegt. wenn direkte Anpassungen vorhanden waren, aneinandergefügt und statistisch inklusive der Wandfragmente aufgenommen. Im Anschluss daran wurde jedes charakteristische Stück einzeln dokumentiert. Die Einzelaufnahme aller morphologischen Elemente führte zur Entwicklung einer eigenen Ada Tepe-Gefäßtypologie, die mittels einer dafür eingerichteten Datenbank auch statistisch auswertbar und digital transformierbar ist. Die verwendeten Terminologien im Deutschen entsprechen den Formen- und Typenbezeichnungen von Kastanas und dem prähistorischen Olynth (z. B. Amphore, Topf), die verwendeten Kurzzeichen beziehen sich auf die im Projekt verwendeten englischen Bezeichnungen (z. B. J1: narrow-mouthed jar/engmundiger Topf). Die massive Fragmentierung der Gefäße ist durchaus mit anderen Siedlungen zu vergleichen, dennoch führt das Fehlen besser erhaltener Profile in Haus 7 nur zu einer schematischen Rekonstruktion der Originalformen. Der Bedeutung der technologischen Charakteristika von Gefäßkeramik versucht die detaillierte Aufnahme von Keramikwaren gerecht zu werden. Diese sollen in einer späteren Projektphase auch petrografisch untersucht werden. Auch hier wird ein etabliertes System makroskopischer Bestimmungen anhand von Härte, Porosität, Bruch, Farbe, Oberflächenbehandlung und Magerung angewendet, das die Definition von Warengruppen erlaubt. ${ }^{13}$ Eine wesentliche Einschränkung im Vergleich dieser Ergebnisse mit anderen Fundorten sind die besonders schlecht erhaltenen ursprünglichen Oberflächen der Scherben, die auf die Lagerungsbedingungen im Boden des Ada Tepe zurückzuführen sind. Das mit diesem Beitrag vorgelegte gesamte Inventar aus Haus 7 des Nordostquartiers beinhaltet einen englischsprachigen Katalog aller 299 signifikanten Keramikfragmente, ${ }^{14}$ wovon eine inhaltlich relevante Auswahl auch auf Tafeln abgebildet ist (Taf. 1-12).

\section{Das Fundensemble aus Haus 7 im Nordostquartier}

Das Inventar aus Haus 7 beinhaltet insgesamt 2295 Keramikscherben (299 charakteristische Stücke und 1996 Wandfragmente), 6 Fragmente von Reibsteinen, 1 Silex-Säge,

12 Horejs 2007a. - Horejs 2010.

13 Horejs 2007a, 52-59. - Horejs 2010.

14 Der Katalog wird der internen Projektsprache folgend auch in diesem Beitrag auf Englisch vorgelegt, um eine kohärente Terminologie für zukünftige Gesamtauswertungen zu gewährleisten.
1 Steinstößel oder -klopfstein sowie ein mögliches zweites Steingerät ohne eindeutige Arbeitsspuren.

\subsection{Keramische Waren}

Die Bestimmung der charakteristischen Stücke in ihren technologischen Eigenschaften aus Haus 7 lässt sich in 14 Warengruppen zusammenfassen, wovon 6 Waren knapp drei Viertel des Inventars repräsentieren. ${ }^{15}$ Mit dieser umfassenden makroskopischen Definition des Inventars soll eine Grundlage für weitere Studien gelegt werden, um zukünftig lokale oder regionale Praktiken in der Keramikproduktion systematisch analysieren zu können. Die Etablierung eines fundortspezifischen Warensystems ermöglicht nicht nur gezielte petrografische Untersuchungen, sondern liefert auch eine direkte Informationsquelle zum Ada Tepe Keramikrepertoire, das hier kurz zusammengefasst werden soll (Taf. 13). Das gesamte Ensemble aus Haus 7 ist handgemacht und wirkt trotz aller Varianten sehr homogen. Mehr als zwei Drittel der Gefäße (67 \%) sind in mittlerer Tonrezeptur bezogen auf Korngröße, Porensichtbarkeit und Magerungspartikel hergestellt. ${ }^{16}$ Weniger häufig sind feine Waren (21\%) und schließlich selten sind Gefäße in grober Rezeptur und Machart (12\%). ${ }^{17}$ Das Warenspektrum zeigt Varianzen der verwendeten Tonpasten und in der Fertigung, was sich beispielweise in den unterschiedlich eingesetzten Brennprozessen der Töpfereien widerspiegelt, die von komplett reduzierend bis durchgehend oxidierend reichen (Taf. 13). Das Farbenspektrum der Brüche und Oberflächen reicht von dunkelgrau, braun, beige bis hellrot und orange. Die Varietäten beinhalten sandige Tonrezepturen, die nur in mittleren und feinen Waren auftreten, sowie organische Magerung, die ausschließlich bei groben Backwannen zu beobachten ist (Taf. 8/1). Alle Waren aus Haus 7 enthalten Glimmer, der auf die Verwendung lokaler oder auch regionaler Tonlagerstätten schließen lässt. Die besonders schlechte Erhaltung der Gefäßoberflächen dürfte den Eindruck der ursprünglichen Gestaltung heute stark verzerren. Zumindest knapp ein Drittel des Ensembles (32 \%) weist noch Spuren eines beigen bis hellbraunen Überzugs auf,

15 Die Erhaltungsbedingungen (Größe, Abrieb, sekundärer Brand und Ähnliches) erlaubten eine technologische Bestimmung von insgesamt 130 Fragmenten aus Haus 7. Die Warengruppen 1, 4, 5, 6, 10 und 12 stellen $72 \%$ dieser definierten Stücke dar. Weniger häufig vertreten sind die Waren 7, 13 und 3 mit insgesamt $20 \%$; selten sind die Waren 2, 9, 11 und 8 mit einem Anteil von nur $8 \%$.

16 Zur detaillierten Beschreibung der verwendeten Kategorien, Größendefinitionen und Maßeinheiten siehe Horejs 2007a, 48-59 und Horejs 2010. In diese Kategorie „mittlerer Waren“ gehören die Ada Tepe Warengruppen 2-7, 9, 10, 13 und 14.

17 Zur Gattung der Feinkeramik gehören die Ada Tepe Gruppen 5 und 12; zur Grobkeramik zählen die Warengruppen 1, 8 und 11. 
Korrelation von Gefäßformen und Warengruppen ( $n=98)$

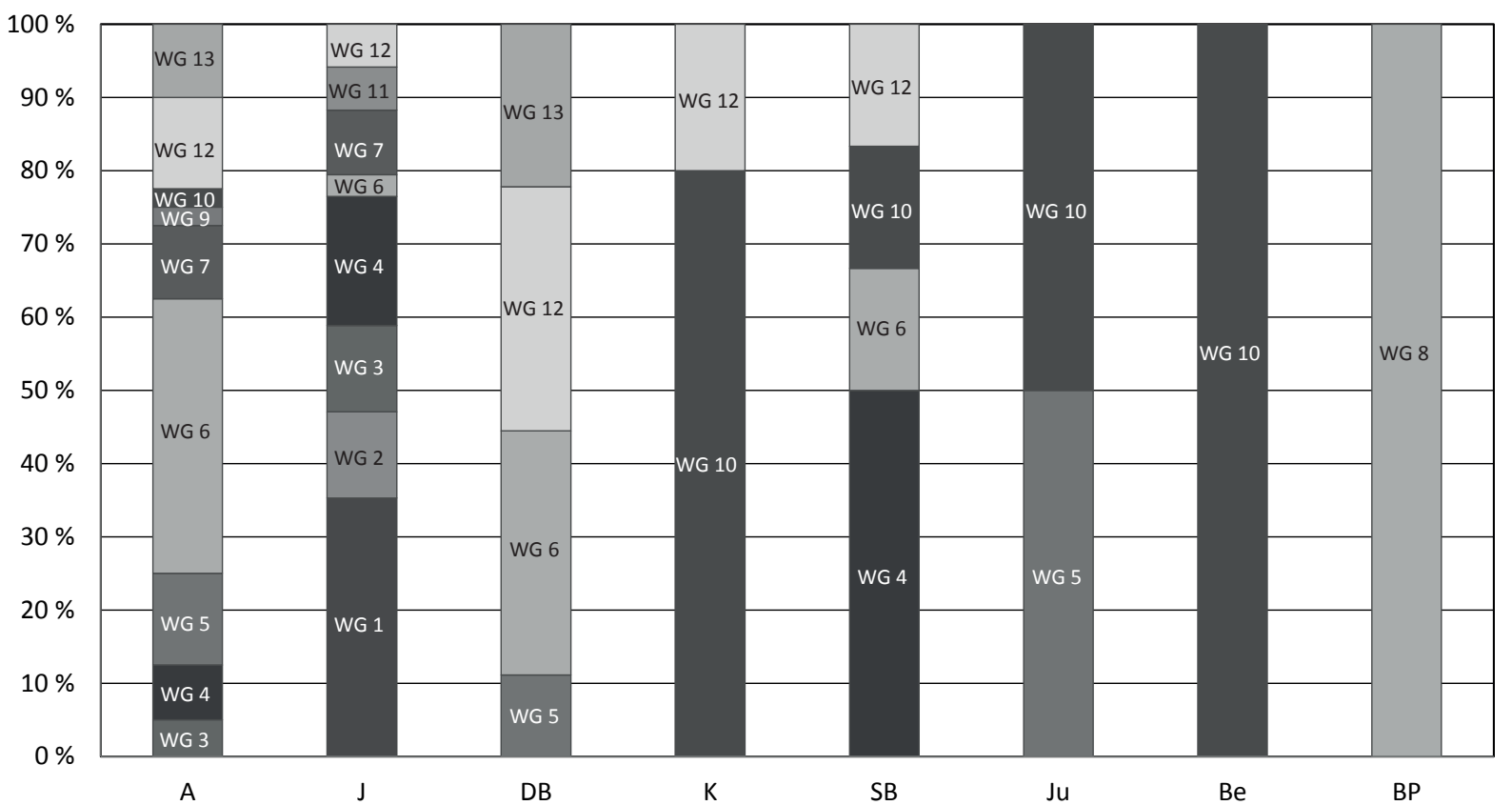

Abb. 3. Korrelation von Gefäßformen und Warengruppen ( $\mathrm{n}=98)$ unter Verwendung der englischen Terminologie. - A: amphora (Amphore). -J: jar (Topf). - DB: deep bowl (Schüssel). - K: kantharos. - SB: shallow bowl (Schale). - Ju: jug or cup (Krug, Tasse). - Be: beaker (Becher). BP: baking pan (Backwanne) (Grafik: M. Röcklinger, OREA).

der die Oberfläche verdichtete und auch optisch vereinheitlichte (Taf. 13: WG 4-5). Weitere Aussagen zu den finalen Arbeitsprozessen in der Gefäßherstellung, wie Polieren und Glätten der Oberflächen sind aufgrund der Erhaltungsbedingungen nur in Einzelfällen möglich. Dennoch zeigt sich auch hier eine vorsichtige Differenzierung in eher grob geglättete bis verstrichene Oberflächen an Grobkeramik und mittleren Waren sowie in feiner geglättete (bis polierte?) Oberflächen an mittleren und feinen Waren.

Die statistische Auswertung aller Waren lässt einen sehr gezielten Einsatz konkreter Rezepturen und Herstellungsdetails für spezielle Gefäßformen annehmen (Abb. 3). So wurde beispielsweise eine feine graue und sandige Ware mit beigem Überzug (Taf. 13: WG 5) ausschließlich für Schüsseln, Kannen oder Amphoren verwendet, während eine sandige dunkelgraue bis schwarze Ware mittlerer Kategorie (Taf. 13: WG 10) vor allem bei der Herstellung von Kantharoi, Schalen, Kannen und Bechern zum Einsatz kam. Ein vergleichbares Produktionsschema ist auch bei der groben dunkelgrauen Ware mit oranger Oberfläche (Taf. 13: WG 1) zu beobachten, die nur für die Herstellung von Töpfen verwendet wurde, was auch auf die grau-beige Ware mittlerer Kategorie (WG 2) zutrifft. Dieser grobe Überblick veranschaulicht einen gezielten Einsatz von Tonrezepturen und Brennprozessen für verschiedene Gefäßkategorien, die auf erfahrene Töpfer schließen lassen. Zudem dürfen wir vermuten, dass die Nutzung von wiederkehrenden Formen in dafür bewusst ausgewählten Keramikwaren auf standardisierte Funktionen schließen lassen. Hier dürften sich bewährte Muster einer etablierten Keramikherstellung widerspiegeln, die sowohl lokale oder regionale Töpfereien wie auch lokale Benutzer inkludieren. Fragen zu regionalen Technologiemustern in der Keramikproduktion sind mangels detaillierter Vorlagen und statistisch verwertbarer Daten derzeit kaum zu beantworten. Einzig das Keramikspektrum der Kamenska Čuka im Strymontal wurde auch in seinen technologischen Waren untersucht und partiell ausgewertet (Abb. 4).$^{18}$ Durchaus typisch dürfte das Verhältnis von feinen, mittleren und groben Waren sowie die damit verbundene Standardisierung der Oberflächenbehandlungen in der gesamten Region der Rhodopen bis Makedonien sein, ${ }^{19}$ wobei sich hier durchaus Unterschiede lokaler Horizonte abzeichnen. So sind beispielsweise im prähistorischen Olynth deutlich mehr feinkeramische Gefäße in Relation $\mathrm{zu}$ den mittleren und groben Kategorien zu beobachten. ${ }^{20}$

18 Stefanovich, Bankoff 1998, 274-275.

19 Z. B. Dragojna: Bozhinova, Jung, Mommsen 2013 oder Kastanas: HochstetTer 1984, 29-37 und Abb. 6. 20 HoREJs 2007a, 49-52. 


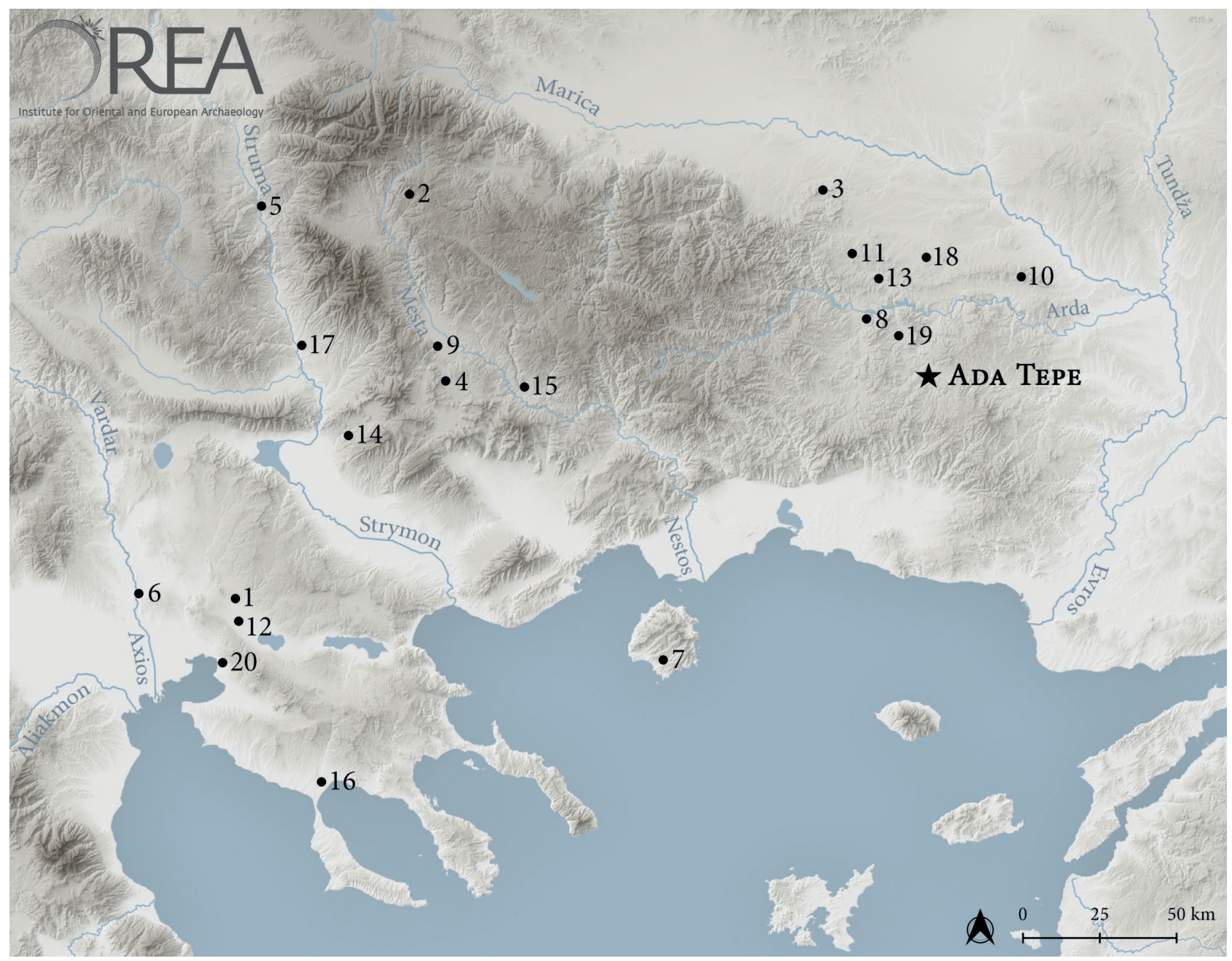

Abb. 4. Im Text erwähnte Fundorte mit vergleichbarer Keramik in Form, Ware oder Dekoration aus der späten Bronzezeit. - 1. Assiros. - 2. Babyak. -3. Dragojna. 4. Exochi. - 5. Kamenska Čuka. -6. Kastanas. -7. Kastri. - 8. Kharman Kaya. - 9. Koprivlen. - 10. Kuš Kaja. -11. Pčelraovo. - 12. Periwolaki. - 13. Perperikon. - 14. Phäa Petra. - 15. Potami. - 16. prähistorisches Olynth. - 17. Sandanski. - 18. Stambolovo. - 19. Tatul. -20. Toumba Thessaloniki (Karte: M. Börner, OREA).

Inwieweit diese Differenzierung auf das gesamte Nordostquartier des Ada Tepe zutrifft oder nur auf das für die vorliegende Studie ausgewählte Haus 7-Ensemble, wird erst die Gesamtauswertung aller Häuser zeigen können.

\subsection{Keramikgefäße}

Das Spektrum der rund 300 signifikanten Stücke umfasst die insgesamt 7 Gefäßformen Amphore, Topf, Schüssel, Schale, Kantharos, Tasse und Becher, die sich aufgrund ihrer Profilierung teilweise weiter gliedern lassen (Abb. 5). Während die Rand- und Lippenformen durchwegs gut bestimmbar sind, ist über die dazugehörige Bodengestaltung kaum eine direkte Aussage möglich. Die Übereinstimmungen der technologischen Waren und Wandstärken der fragmentierten Böden mit Gefäßoberteilen zeigen, dass vor allem bei Amphoren und Töpfen Flachböden mit teilweise verstärkter Standfläche üblich sind (Taf. 1/1; 3/2-5; 4/2-3; $5 / 5 ; 6 / 4$ ). Wesentlich seltener sind Standfüße (Taf. 10/5; 12/6), die wohl zu Schalen und Schüsseln sowie kleineren Amphoren oder Töpfen gehören dürften. Die für den Plovdiv-Čerkovna-Horizont typische Randform einer nach außen verstärkten Lippe ist auch im Ada Tepe Haus 7 festzustellen (Lippenform B und H: Taf. 1/1-2; 2/4, 6; 3/8; $6 / 1 ; 7 / 1-3 ; 8 / 3,5 ; 10 / 6) .{ }^{21}$ Im Verhältnis zu anderen Gestaltungen der Mündungen ist diese Verstärkung aber, anders als in Dragojna, nicht dominierend, was sowohl durch ein

21 Diese von E. Bozhinova postulierte These scheint durchaus überzeugend (Bozhinova, Jung, Mommsen 2013, 53), wie ein Blick in die Spektren anderer Fundorte der Spätbronzezeit in den Rhodopen vermuten lässt: z. B. Kuš Kaja: Popov 2009, Abb. 10-11 oder Babyak: Dimitrova 2014, Abb. 3-7. 


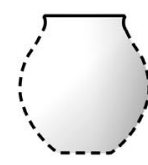

A1 - Amphore mit zylindrischem Hals - konisch

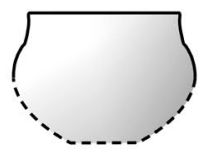

DB2- Schüssel mit zylindrischem Hals<smiles>C1CCCCCCC1</smiles>

A2 - Amphore mit konischem Hals - ausbiegend

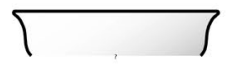

SB1 - S-förmige Schale<smiles>C1CCCCCCCCCCC1</smiles>

A3 - Amphore mit zylindrischem Hals - gerade

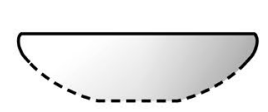

SB2 - Schale mit leicht einziehendem Rand

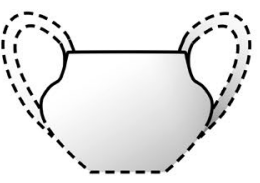

K1 - Kantharos mit zylindrischem Hals - kugelförmig

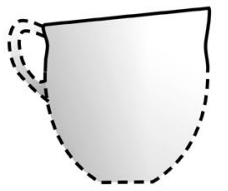

Cu1 - Tasse mit schrägem Mundsaum

\section{- konisch}

J3 - Topf mit zylindrischem Hals - eiförmig

J4 - Engmundiger Topf - konkav

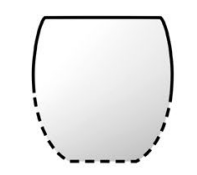

Be1 - Engmundiger Becher

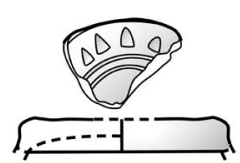

L1A - Einfacher Deckel - dekoriert

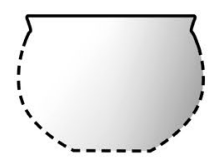

DB1- Schüssel mit trichter-

förmigem Hals

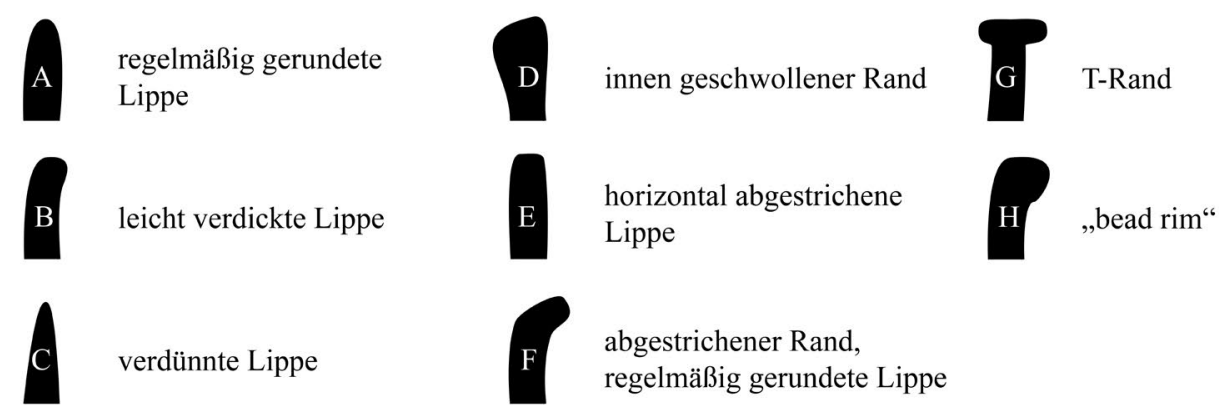

Abb. 5. Schematische Übersicht aller in Haus 7 vorkommenden Gefäßformen und Typen sowie Lippenformen. Die unterbrochenen Linien sind hypothetisch rekonstruierte Profile (Illustration: M. Röcklinger, OREA). 


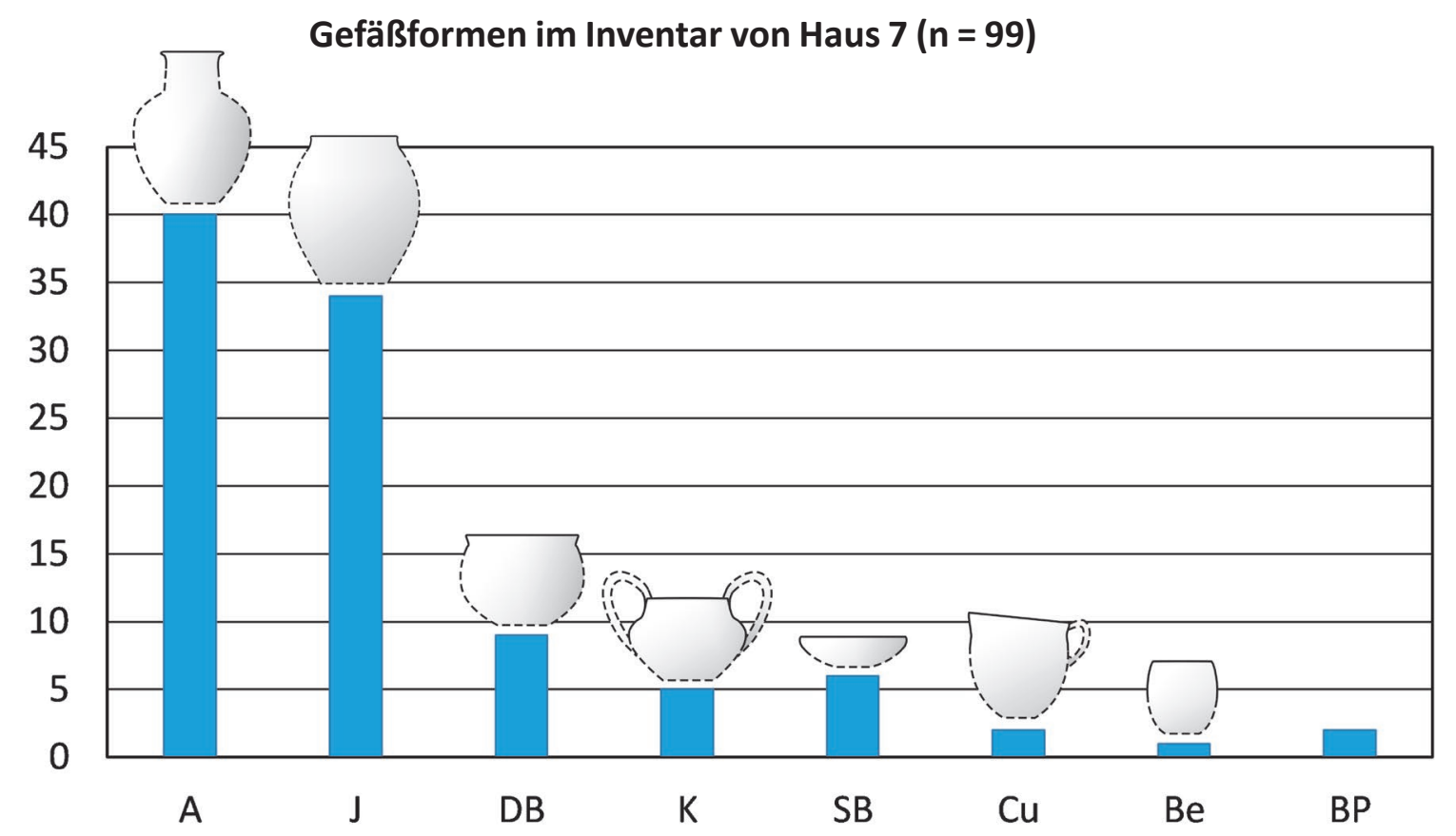

Abb. 6. Absolute Häufigkeit aller Gefäßformen im Inventar von Haus 7 ( $n$ = 99) des Ada Tepe (Grafik: M. Röcklinger, OREA).

lokales Charakteristikum als auch chronologisch begründet sein könnte. ${ }^{22}$ Mehr als 3/4 der Gefäße (78 \%) sind nicht verziert, was sowohl chronologische, regionale oder auch funktionale Gründe haben könnte. ${ }^{23}$ In der Gestaltung einzelner Gefäßtypen lässt sich ein wiederholendes Schema feststellen, das sich durchaus mit anderen Siedlungen der weiteren Region vergleichen lässt. Zunächst seien hier die Amphoren mit konischem Hals (A2) sowie mit geradem Zylinderhals (A3) herausgegriffen (Taf. 1/3; 3/1; 4/1; 5/3; $8 / 2 ; 11 / 3-4)$, die in der gesamten Spätbronzezeit von Zentralmakedonien und der Chalkidiki (Perivolaki, Toumba Thessaloniki, prähistorisches Olynth) sowie Ostmakedonien (Exochi, Potami, Phäa Petra, Kastri) bis in die westlichen (Sandanski, Koprivlen, Babyak) und östlichen Rhodopen (Dragojna, Kuš Kaja) und zum Strymontal (Kamenska Čuka) gerne verwendet werden (Abb. 5). ${ }^{24}$ Amphoren sind die häufigsten Gefäße im Ensemble von Haus 7 (Abb. 6)

22 Bozhinova, Jung, Mommsen 2013, 53, 63-65 zum Problem der absoluten Datierung des Plovdiv-Čerkovna-Horizonts und einer Verankerung der Subphasen innerhalb der späten Bronzezeit in den Rhodopen.

23 Die Statistik basiert auf Randfragmenten, um keine statistische Verzerrung durch dekorierte Wandscherben zu bewirken.

24 Grammenos 1981, 34, 45. - Koukouli-Chrysanthaki 1992, 706. - Kisjov 1994, Abb. $3 / 8$ und 12. - Stefanovich, Bankoff 1998, Abb. 29-30. - Valla 2000, 108. - Horejs 2007a, 154-157. - Popov 2009, Abb. 10. - Bozhinova, Jung, Mommsen 2013, 54-55. Dimitrova 2014, Abb. 5. und treten überwiegend unverziert auf. Lediglich ein Exemplar dürfte eine für den Ada Tepe durchaus charakteristische dekorierte Amphore sein (Taf. 5/2), wie wir sie vor allem von anderen Siedlungsarealen des Hügels kennen. ${ }^{25}$ Die zusammengefügten Wandfragmente aus Haus 7 zeigen die typischen Sonnenmotive in Kombination mit hängenden Mehrfachdreiecken in Furchenstichtechnik. ${ }^{26}$ Die Haus 7-Amphoren sind durchwegs aus feinen oder mittleren Waren hergestellt und weisen - sofern erhalten - geglättete Oberflächen auf, was sich mit den Exemplaren sowohl von der Kamenska Čuka als auch von Kastanas und dem prähistorischen Olynth vergleichen lässt. ${ }^{27}$

Die neben den Amphoren zweite dominierende Gefäßgruppe des Hausinventars sind Töpfe (jars), die in überraschend großer Vielfalt zu beobachten sind. Sie treten als engmundige Töpfe mit gerader oder konischer Mündung (J1, J2), konkavem Oberteil (J4) oder hohem eiförmigen Körper mit zylindrischem Hals (J3) auf (Taf. 1/2, 6; 2/4, 7; $4 / 4,6 ; 5 / 1 ; 6 / 2 ; 7 / 1-3 ; 8 / 4 ; 9 / 4 ; 10 / 3,6)$. Maximal 15 Exemplare aller vier Typen sind mit groben Eindrücken oder

25 Nikov 2017, Abb. 1-2 und Kat. Nr. 118.

26 Zur Keramikverzierung auf dem Ada Tepe siehe Popov et al. 2015, Abb. 17 und Nikov 2017 mit Abb. 2-3. - Zu den Ostrhodopen Nechrizov, Cvetkova 2017, bes. 53-54 und Abb. 3. - Weitere Beispiele aus Tatul und Perperikon: Leshtakov 2009, Abb. 8-9. Kisjov 1994, Abb. 14.

27 Hochstetter 1984, 42-44. - Stefanovich, Bankoff 1998, 275. - Horejs 2007a, 155-157. 

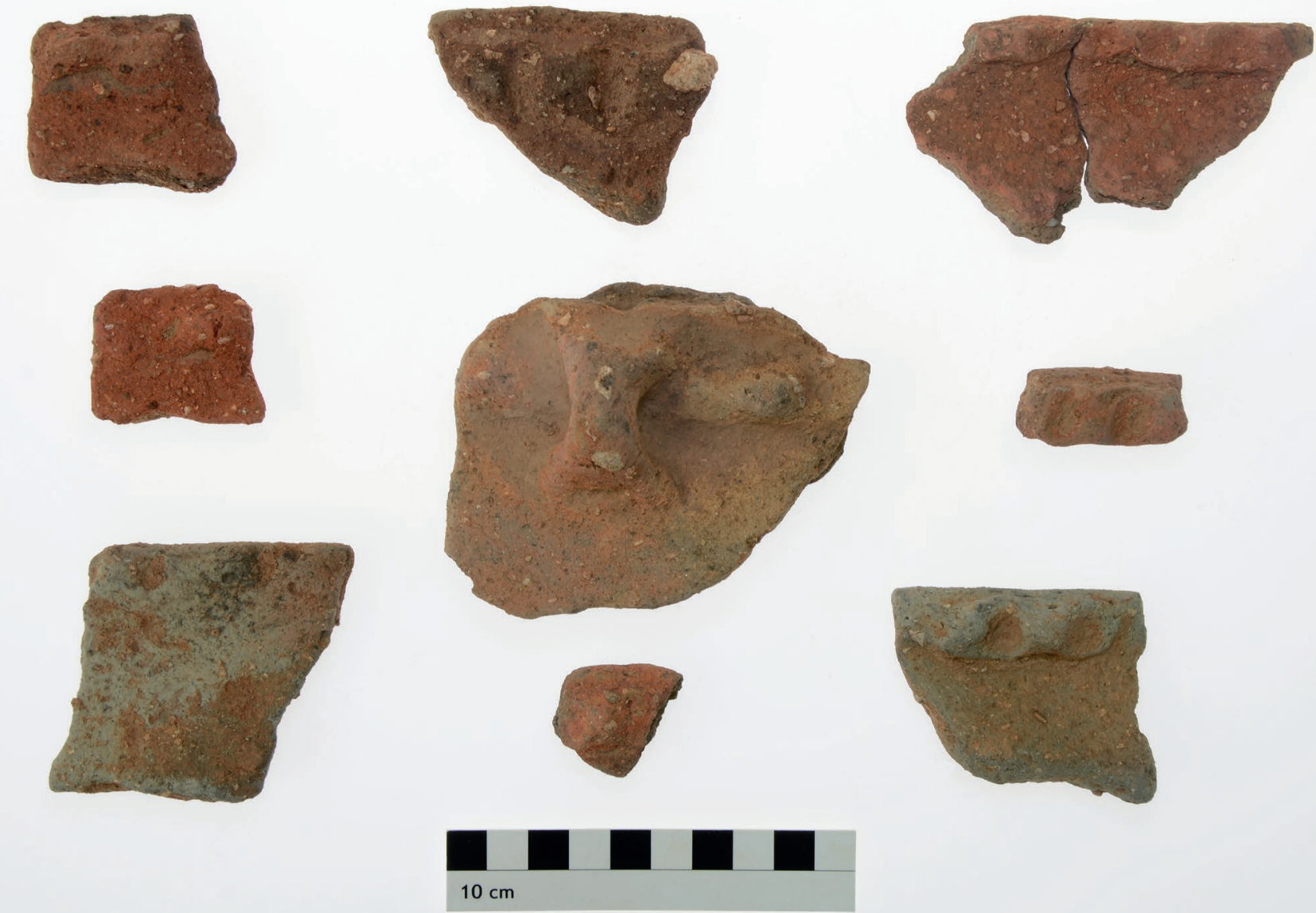

Abb. 7. Beispiele verzierter Topffragmente aus Haus 7 des Ada Tepe (Foto: F. Ostmann, OREA).

Fingertupfenleisten verziert, die wohl auch als horizontale Verstärkung der Mündung und seltener auch auf der Gefäßmitte eingesetzt wurden (Abb. 7 und Taf. 7/1; 10/3; 12/3). Die bereits erwähnten Flachböden dürften teilweise die dazugehörigen Basen dieser Töpfe darstellen. Töpfe sind als einzige Form auch in groben Waren produziert worden (WG 1), die sich zusätzlich zu ihrer großen Porosität auch durch eine besonders grobkörnige und dichte Magerung auszeichnen (Taf. 13: WG 1). Daneben gibt es auch Exemplare, die der mittleren Warenkategorie angehören, vereinzelt wurden sie auch feinkeramisch hergestellt, was wohl direkt mit ihrem Einsatz für Vorrat von Flüssigkeiten oder festen Bestandteilen oder auch Nahrungsverarbeitung zu tun haben dürfte. Auch das Spektrum der Töpfe lässt sich in einem regionalen Kontext der Rhodopen verankern, ${ }^{28}$ wenn auch nicht alle Typen entsprechend häufig oder weit

28 Vgl. die spätbronzezeitlichen Töpfe in Babyak: Dimitrova 2014, Abb. 6-7 und Kuš Kaja: Popov 2009, Abb. 10. verbreitet sind. Inwieweit hier eventuell auch ein lokaltypischer Horizont sichtbar wird, werden erst die Studien zur gesamten Keramik des Nordostquartiers definitiv zeigen können. Das Verhältnis von wenigen verzierten zu wesentlich mehr unverzierten Töpfen könnte jedenfalls regional charakteristisch sein, auch wenn statistische Datenvorlagen zu Siedlungen in den Rhodopen bisher fehlen. ${ }^{29}$ Die Dominanz von engmundigen Formen innerhalb der Töpfe scheint hingegen eher lokaltypisch zu sein und hängt möglicherweise direkt mit ihrer Funktion in Haus 7 zusammen.

Wesentlich seltener als die beiden hohen und geschlossenen Amphoren und Töpfe (Anteil von 76 \%) sind Schüsseln, Schalen, Kantharoi, Tassen und Becher, die gemeinsam nur rund $20 \%$ im gesamten Inventar ausmachen. Dementsprechend gering ist auch ihre Typenvielfalt und technologische Varianz. Schüsseln (deep bowls) kommen mit trichterförmi-

29 Horejs 2007a, 126-145. - Bozhinova, Jung, Mommsen 2013, 60-61. 
gem oder zylindrischem Hals vor (Taf. 2/3; 4/5; 6/1; 10/4; $11 / 5)$, zumeist mit regelmäßiger gerundeter Lippenform. Die 9 Exemplare aus Haus 7 sind aus feinkeramischen Waren (WG 5, 12) und Waren der mittleren Kategorie hergestellt (WG 6, 13) (Abb. 3); sie sind alle aus sandgemagerten Tonen hergestellt, haben teilweise einen beigen Überzug und sind von graubrauner, beiger oder rotoranger Farbe.

Insgesamt vier Exemplare von Schalen (shallow bowls) sind im Repertoire von Haus 7 vertreten: s-förmig profiliert oder mit leicht einziehender Mündung (Taf. 2/5; 9/3; 7/4; 11/2). Sie sind wie Schüsseln nur aus Waren feiner oder mittlerer Kategorien hergestellt und sind ebenfalls teilweise mit beigem Slip überzogen (WG 4). Der Fragmentierungsgrad von Schalen und Schüsseln aus Haus 7 erlaubt keine Aussage zu möglichen Handhaben oder Böden. Lediglich eine mit Dreieck und Spiralmotiv in Furchenstichtechnik verzierte Schale weist einen Henkelansatz an der Mündung auf (Taf. 7/4). Es muss offen bleiben, ob es sich um einen möglichen Wishbone-Henkel handelt, wie er für die Spätbronzezeit Makedoniens überaus charakteristisch ist und zumindest vereinzelt auch in den Rhodopen bzw. der Plovdiv-Čerkovna-Gruppe zu beobachten ist. ${ }^{30}$ In ihrer Gestaltung passen die Schalen und Schüsseln sehr gut in das regionale Spektrum. So sind die s-förmigen Schalen sowohl in den Rhodopen als auch in Westmakedonien, auf der Chalkidiki, in Ostmakedonien und der Bucht von Volos typisch. ${ }^{31}$ Als einige der wenigen absolut datierten Vergleiche ist das Vorkommen dieses Schalentyps im prähistorischen Olynth zwischen MH III/SH I und SH IIIA1 (16./15. Jh. v. Chr.) erwähnenswert, ${ }^{32}$ wenn auch der Typ in den Rhodopen, in Ostmakedonien und dem Strymontal durchaus länger in Gebrauch gewesen sein dürfte. Die Schüsseln mit trichterförmigem Rand lassen sich ebenfalls gut im lokalen bis regionalen Milieu einbetten, wo sie verziert und unverziert von den Rhodopen bis nach Westmakedonien und Thessalien vorkommen. ${ }^{33}$

Der für die Region so typische Kantharos mit kugelförmigem Bauch und Zylinderhals ist mit mindestens fünf Exemplaren im Inventar von Haus 7 vertreten (Taf. 1/4-5; 3/6; 4/7). An den besser erhaltenen Stücken sind die für die Rhodopen und Zentral- bis Ostmakedonien charakteristischen

30 Horejs 2007a, 108 und Abb. 53.

31 Grammenos 1981, 35, 44. - Koukouli-Chrystanthaki 1992, Taf. 9 und 10/91. - Kisjov 1994, Abb. 2-4. - Stefanovich, BankofF 1998, Abb. 28. - Horejs 2007a, 102 und Abb. 46. - Dimitrova 2014, Abb. 4. - Nechrizov, Cvetrova 2017, 54 und Abb. 3.

32 Horejs 2007a, 101-103 und Abb. 45.

33 Kisjov 1994, Abb. 2, 4, 7, 9 und 13. - Horejs 2007a, 113 und Abb. 58 mit Ergänzungen bei Bozhinova, Jung, Mommsen 2013, 56-57 und Taf. 4. - Popov 2009, Abb. 10. - Dimitrova 2014, Abb. 4.
Dekorationsmotive von triangulären Linienbündeln, Doppelspiralen und Horizontallinienreihen erhalten (Taf. 1/45). ${ }^{34}$ Sie sind überwiegend aus einer sandigen dunkelgrauen Ware hergestellt, die der mittleren Kategorie angehört (WG 10). Weitere verzierte Wandfragmente ohne direkte Anpassungen lassen sich aufgrund ihrer Form, Profilierung und Wandstärke den Kantharoi sehr wahrscheinlich zuweisen (Taf. 6/6; 7/5; 9/5-6; 10/1-2; 11/1; 12/2).

Schließlich sei noch eine Gruppe von Bechern bzw. Tassen (beakers, cups) anhand von drei Exemplaren kurz vorgestellt. Ein mit Furchenstich dekoriertes offenes Gefäß lässt sich nicht mit Sicherheit den Tassen zuweisen (Taf. 3/7), lässt uns das aber aufgrund des leicht hochgezogenen Mündungssaums vermuten. ${ }^{35}$ Ein Becherfragment mit dünner Randlippe, leicht eingezogener Mündung und blattförmigem Furchenstichdekor (Taf. 12/1) entspricht den im Plovdiv-Čerkovna-Horizont und den Rhodopen typischen einhenkeligen Trinkgefäßen, auch wenn an unserem Exemplar keine Handhabe erhalten ist. ${ }^{36}$ Das Stück ist aus derselben Ware wie die verzierten Kantharoi gefertigt (WG 10). Schließlich lässt sich noch ein drittes verziertes Exemplar in diese Kategorie von Tassen bzw. Bechern einordnen (Taf. 9/1), das ebenfalls in dieser Ware produziert wurde.

\subsection{Funktionale und kontextuelle Interpretationen}

Die funktionale Interpretation von Keramikgefäßen des Ada Tepe kann derzeit ausschließlich über morphologische, technologische und kontextuelle Indizien erfolgen, da Inhaltsanalysen bisher (noch) nicht durchgeführt wurden und aufgrund der sehr schlechten Erhaltungsbedingungen insgesamt auch nicht sehr vielversprechend erscheinen. Auch sind komparative Studien für die gesamte Region aufgrund fehlender Daten derzeit nicht möglich, einzig aus Kuš Kaja wurde jüngst ein Gefäß mit organischen Resten publiziert. Es handelt sich um ein tiefes Gefäß mit Resten aus Hirse, Gerste und einem geringeren Anteil an Leinsamen, die sich als getrockneter Brei erhalten haben. ${ }^{37}$ Dieser einzige direkte Nachweis eines Gefäßinhaltes in der Region bestätigt zumindest die theoretischen Grundlagen unserer funktionalen

34 Vgl. z. B. Koukouli-Chrysanthaki 1992, 480-481. - Kisjov 1994, Abb. 6 und 14. - Horejs 2007b, Taf. 76. - Nechrizov, CvetKOva 2017, 54 und Abb. 3.

35 Solcherart Tassen (mitunter auch als Krüge bezeichnet) finden sich in der gesamten Region, siehe z. B. Kisjov 1994, Abb. 5. - STEFANovich, Bankoff 1998, Abb. 25. - Leshtakov 2009, Abb. 9. - Popov 2009, Abb. 11.

36 Kisjov 1994, Abb. 5. - Nechrizov 1995, 314-315. - Bozhinova, Jung, Mommsen 2013, 58 und Taf. 5 mit weiteren Parallelen. - Popov 2016, Abb. 2-3.

37 Popov et al. im Druck. 


\section{Lagerung:}
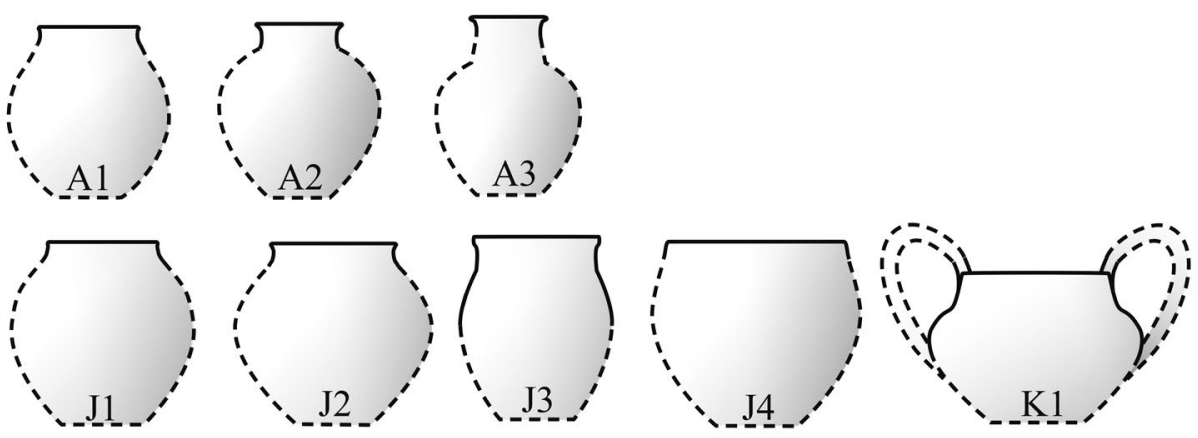

Zubereitung:? 2 Backwannen

\section{Konsum:}
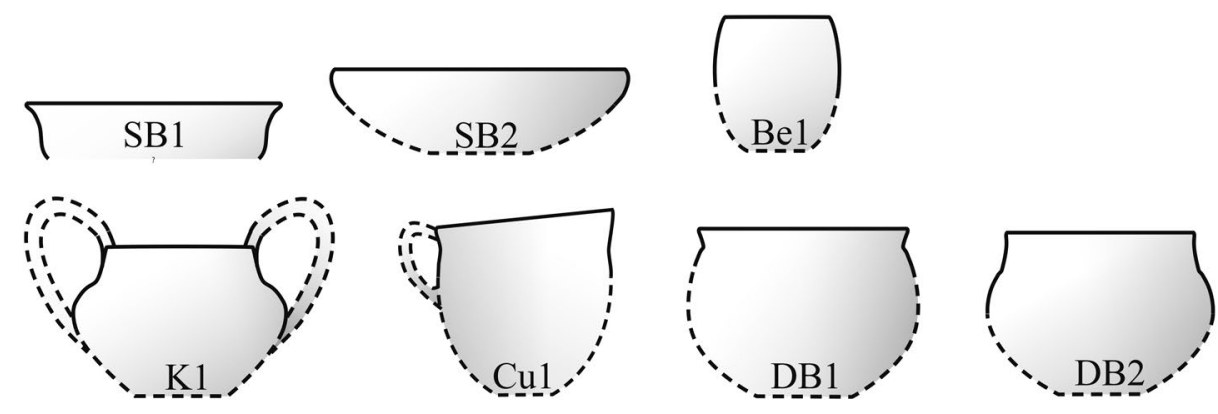

Abb. 8. Gliederung der Gefäßtypen aus Haus 7 nach ihrer wahrscheinlichen Verwendungskategorie nach RIEMER 1997 (Grafik: M. Röcklinger, OREA).

Analysen, die auf Form, Technologie und Kontext beruhen. Der grundlegenden Einteilung H. Riemers folgend, lassen sich die drei Nutzungskategorien Bevorratung, Zubereitung und Darreichung/Konsumierung definieren. ${ }^{38}$ Dabei ist natürlich zu berücksichtigen, dass Gefäße wohl auch in mehrfacher Hinsicht genutzt wurden und als praktisch eingesetzte Gegenstände multifunktional sein können. Dennoch zeigt die gezielte Produktion wiederkehrender Formen in annähernd standardisierten technologischen Waren, dass der Bedarf für eine konkrete Nutzung angenommen werden kann.

Ausgehend von dieser Prämisse, lassen sich grundsätzlich im gesamten Keramikinventar von Haus 7 vor allem zwei Funktionen erkennen, die mit den Kategorien Lagerung und Konsum von Lebensmitteln umschrieben werden können (Abb. 8). In den Bereich von Lagerung flüssiger wie auch fester Nahrungsmittel gehören vor allem Amphoren

38 Riemer 1997, 117-131. und Töpfe, die sich durch ihre hohe und engmundige Form dafür bestens eignen. Die sicherlich je nach Bedarf genutzten Gefäße für Flüssigkeiten (Wasser, gegorene Getränke und Ähnliches) und Nahrung (Getreide, Früchte, Gemüse, Fleisch etc.) sind technologisch in entsprechend größerer Varianz sowie verschiedenen Größen hergestellt worden (Abb. 9). Die nur selten erhaltenen Gefäßoberflächen würden hier vermutlich auch eine Differenzierung (Überzug und Glättung) widerspiegeln. Da sich an den vermutlich zuordenbaren Böden sowie Wandfragmenten kaum sekundäre Brandspuren wie Schmauchspuren oder die Bildung von Rissen durch Feuereinwirkung finden, wurden sie vermutlich nicht zum Erhitzen über dem Feuer verwendet. Umso erstaunlicher ist in diesem Zusammenhang das Fehlen von Pyraunoi (tragbare Feuerherde), die zwar vor allem im Karpatenbecken nördlich der Donau sowie in Makedonien typisch sind, aber möglicherweise bis in die Rhodopen verbreitet waren, was noch nicht restlos geklärt 
Korrelation der Warengruppen mit den Gefäßfunktionen ( $n=98)$

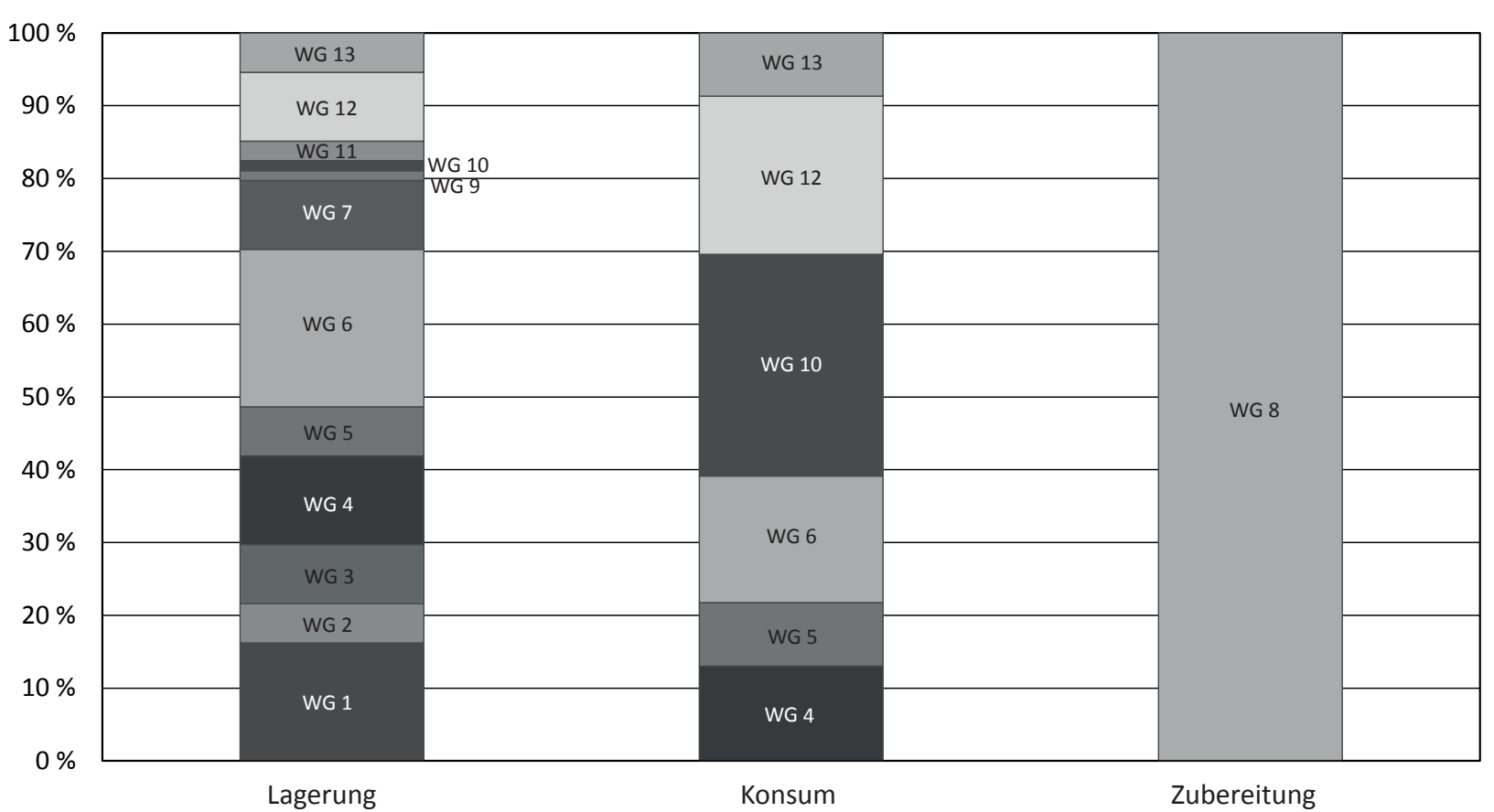

Abb. 9. Korrelation der einzelnen Warengruppen mit den Kategorien der Verwendung von Typen ( $\mathrm{n}=98)$ (Grafik: M. Röcklinger, OREA).

ist. ${ }^{39}$ Einzig die Fragmente von vermutlich zwei verschiedenen Backwannen mit eindeutigen Schmauchspuren lassen auf Erhitzen in Form von Backen bzw. Kochen schließen. Die sehr charakteristische Ware dieser Backwannen aus porösem, organisch gemagertem Ton und ohne jegliche Verdichtung der Oberflächen (Taf. 8/1) lässt hier eher auf die Herstellung von festen Lebensmitteln (Brot, Getreidebrei oder Ähnliches) schließen..$^{40}$

Schließlich lässt sich die Aktivität von Lebensmittelkonsum in flüssiger und fester Form in der Gruppe der Schalen und Becher bzw. Tassen annehmen. Dazu gehören wohl auch die tiefen Schüsseln, die sich zum Darreichen ebenso eignen wie zum Konsum von Nahrung. Die

39 Fischl, Kiss, Kulcsár 2001. - Horejs 2007a, 148-153 mit Abb. 97. - Zu möglichen spätbronzezeitlichen Pyraunoi in Dragojna siehe Bozhinova, Jung, Mommsen 2013, 60. - Zur Frage von unterschiedlichen Kochtraditionen in der Spätbronzezeit siehe HorEJS 2005.

40 Vgl. hier beispielsweise die Backwannen von Kastanas: НоснSTETTER 1984, 164-168 und Taf. 44/6 und dem prähistorischen Olynth: Horejs 2007a, 177, die an beiden Orten in derselben Technologie hergestellt sind, auch wenn sie in Olynth auf die frühe SBZ beschränkt sind und in Kastanas vor allem in der Eisenzeit typisch werden. In beiden Fällen liegt die Begründung wohl eher am limitierten Ausschnitt der Ausgrabungen als an tatsächlichen chronologischen Beschränkungen. technologischen Details der Keramik bekräftigen diese Vermutung, da diese Formen aus Haus 7 ausschließlich in Waren feiner und mittlerer Kategorie vorkommen (Abb. 8). Dieses Trink- und Essgeschirr ist zudem auch oft verziert (Taf. 3/1; 7/4-5; 9/1;12/1), was seine Nutzung als Tafelservice zusätzlich unterstützt.

Die Rolle der Kantharoi mag vielfältig sein, da sie sich sowohl zur Lagerung von speziellen Flüssigkeiten (z. B. Öle ${ }^{41}$ ) ebenso eignen wie zur Konsumierung von Flüssigkeiten (abhängig von ihrer Hals- und Lippenform), weshalb sie in beiden Kategorien angeführt sind. Verzierte Kantharoi gehören zum Standardrepertoire in spätbronzezeitlichen Siedlungen in den Rhodopen und darüber hinaus, ${ }^{42}$ und

41 Andreou et al. 2013.

42 Beispielsweise eventuell in Kuš Kaja: Popov 2009, Abb. 11 (Kantharos verziert?), Dragojna: Bozhinova, Jung, Mommsen 2013, Taf. 3/1-4, Višegrad/Kharman Kaya: Nechrizov 1995, Abb. 2/5-6, Koprivlen: Alexandrov 2002, Abb. 14/6, 8; 23/5, Peperikon: NeCHrizov, Cvetrova 2017, Abb. 3, 8, evt. Stambolovo: Nechrizov, Cvetkova 2017, Abb. 3, 14, Pčelarovo: Nechrizov, Cvetkova 2017, Abb. 3, 4. - Zu ihrer Verbreitung außerhalb der Rhodopen in auch unverzierter Form siehe HäNSEL 1976 und HäNSEL 1982 mit Ergänzungen bei Horejs 2007a, 121 und Abb. 66 (unverzierte Kugelkantharoi). - Die Rolle von verzierten Kantharoi in den Rhodopen ist noch zu klären, da die wenigen Publikationen proportional mehr dekorierte Exemplare zeigen dürften als statistisch relevant. 


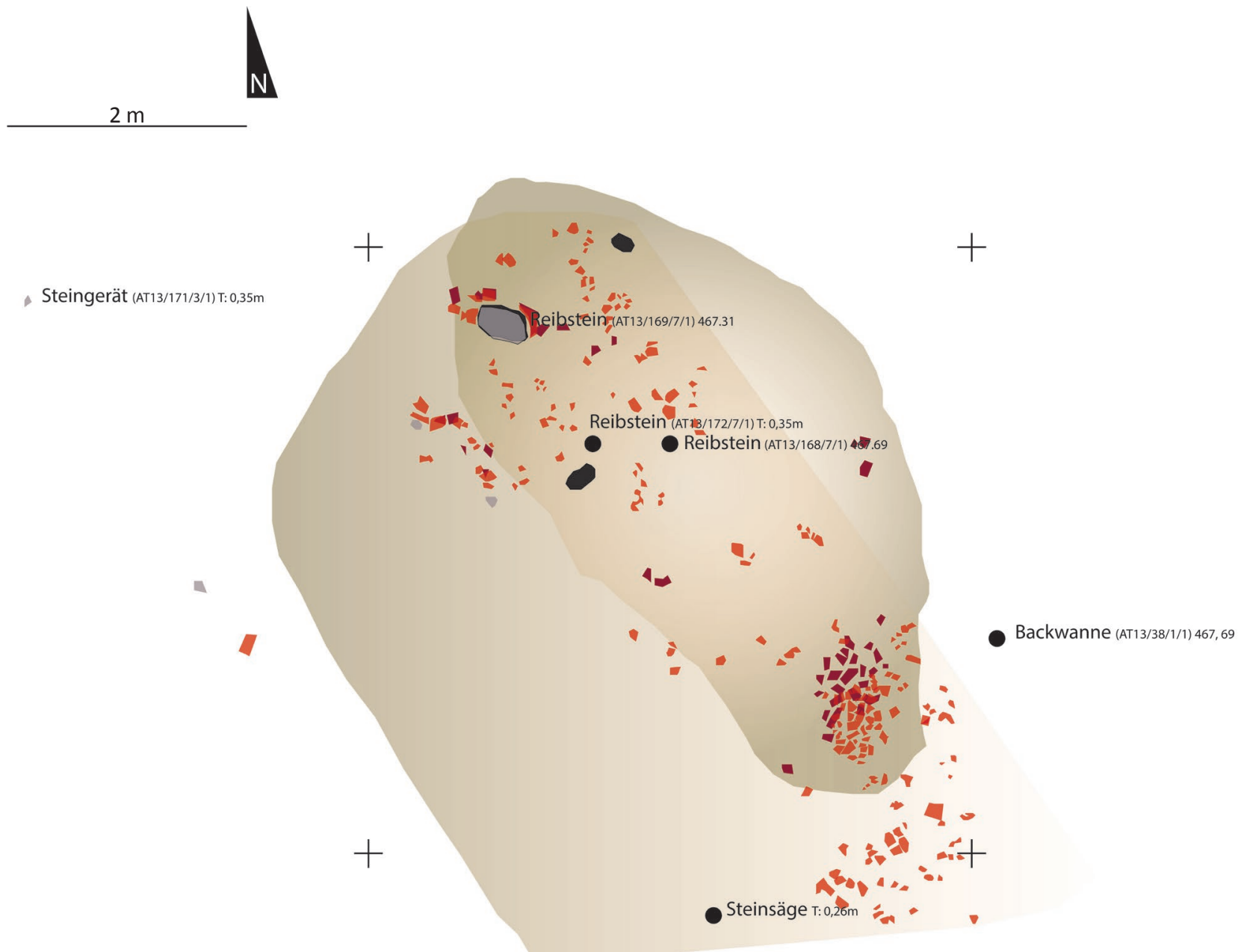

Reibstein (AT13/174/7/1)T:0,50m

Reibstein (AT13/170/7/1) T:0,35m

Steingerät (AT13/173/3/1) T: 0,40m

Abb. 10. Schematischer Plan der Fundverteilung in Haus 7 des Ada Tepe. Horizontale Lage und räumliche Ausdehnung = beige; Keramik (Layer 2$)$ = orange; Keramik $($ Layer 3$)=$ magenta; Steinartefakte = grau (Plan: L. Burkhardt, OREA nach E. Nikolova, NAIM mit freundlicher Genehmigung von $\mathrm{H}$. Popov, NAIM).

müssen daher auch mit siedlungstypischen Tätigkeiten in Verbindung stehen. Ihre mitunter besonders qualitätsvolle Produktion (dünner und regelmäßiger Gefäßaufbau, polierte Oberflächen, gleichmäßige Brenntemperaturen) sowie ihre - zumindest in den Rhodopen - charakteristische aufwändige Verzierung ${ }^{43}$ - mag auf eine sehr spezielle Nutzung im gesellschaftlichen Kontext schließen lassen.

Die statistische Auswertung dieser Ergebnisse zeigt sehr deutlich, dass wir in Haus 7 vor allem Lagerung und

43 Koukouli-Chrysanthaki 1992, 480-481. - Nechrizov, CvetKova 2017. - NiKov 2017.
Speicherung von Nahrung und Flüssigkeiten vermuten dürfen, da diese Gruppe dominiert (Abb. 6 und 8), gefolgt von Konsum bzw. Darreichung von Lebensmitteln. Kochen dürfte hingegen innerhalb von Haus 7 kaum stattgefunden haben, sondern ist außerhalb der Struktur zu vermuten. Diese Annahme bestätigt auch die räumliche Analyse der Grabungsdaten (Abb. 10). Die Gefäßfragmente stammen aus dem Inneren mit einer klaren Kulmination im nördlichen und südlichen Bereich der Struktur. Es gibt keine Nachweise von Feuerstellen oder anderen pyrotechnischen Installationen im Hausinneren, das offenbar vor allem zur Lagerung genutzt wurde. Die außerhalb von Haus 7 


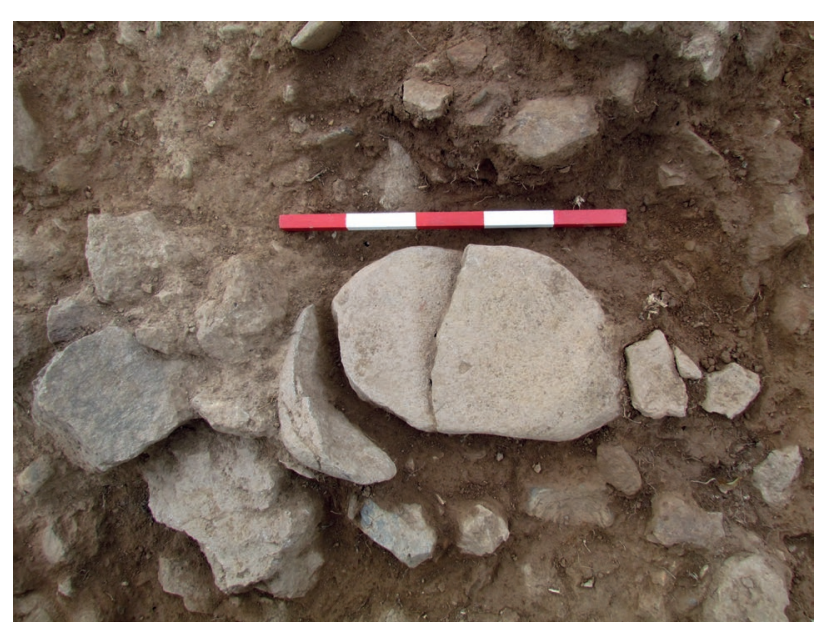

Abb. 11. Reibstein (AT 13/169/7/1) im nördlichen Bereich von Haus 7, Aufnahme der Grabungssituation (Foto: NAIM).

gefundenen Reste von Backwannen lassen vorläufig auf Kochen bzw. Backen im Freien schließen, auch wenn erst die Auswertung aller Hausinventare und Außenbereiche eine endgültige Interpretation erlauben wird. Die kleine Gruppe an Konsumgefäßen könnte entweder auf direktes Trinken und Essen in Haus 7 hinweisen, ebenso gut aber auch nur die Aufbewahrung dieser Gefäße widerspiegeln. Da es aber Hinweise auf Lebensmittelverarbeitung durch andere Fundobjekte wie Reibplatten (siehe unten) gibt, scheinen nicht nur passive Speicherung, sondern auch aktive Tätigkeiten stattgefunden zu haben. Insofern würden die Reste von Konsumgefäßen durchaus in das Aktivitätsbild passen. Wie eingangs erläutert, spiegelt das Ensemble an Funden aus Haus 7 keinen einzelnen Zeitpunkt einer Deponierung im Sinne eines geschlossenen Kontexts bei der Zerstörung des Baus wider. Dennoch lassen die Deponierungsprozesse (kurze Nutzungszeit, Versiegelung durch spätbronzezeitlichen Haldenschutt) darauf schließen, dass die Scherben auf die wenigen Jahrzehnte (Jahre?) der Hausnutzung Rückschlüsse erlauben. Eine spätere Verunreinigung durch Müllablagerung oder Ähnliches ist aufgrund der Versiegelung und Überlagerung sehr unwahrscheinlich.

\subsection{Geräte und Werkzeuge}

Sowohl komplett erhaltene als auch gebrochene Reibsteine (Unterlieger und Läufer) inner- und außerhalb von Haus 7 lassen verschiedene Tätigkeiten vermuten (Abb. 11). Diese universalen Mahlgeräte konnten einerseits zum Zerkleinern oder Pochen von Mineralien eingesetzt werden, stehen im Siedlungskontext aber zumeist in funktionaler Verbindung zu Getreideverarbeitung (Taf. 14-15). Auch wenn derzeit noch keine Oberflächenanalysen der Reibsteine aus Haus 7 vorliegen, so lässt ihre unmittelbare Nähe zu den

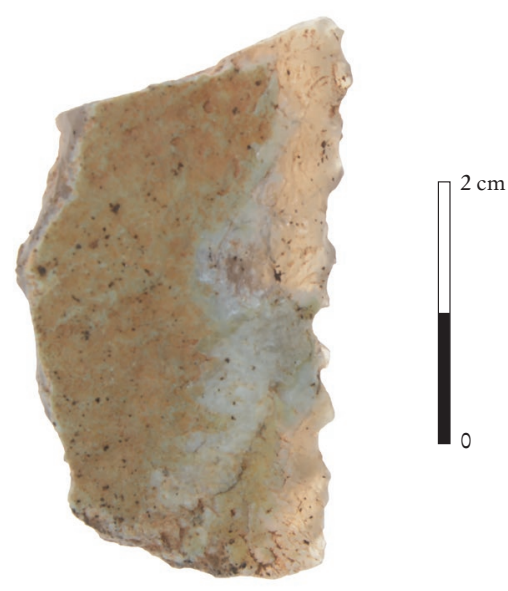

Abb. 12. Silex-Säge (AT 12/54/4/1) aus dem südlichen Innenbereich von Haus 7 (Foto: F. Ostmann/OREA).

Vorratsgefäßen eine nahrungsbezogene Nutzung vermuten. Eine kleine Silex-Säge aus dem Bereich der südlichen Gefäßkulmination weist ebenfalls auf häusliches Handwerk (schneiden, sägen) hin (Abb. 12). Zwei Reibsteine und ein Klopfstein im südlichen Außenbereich von Haus 7 illustrieren (Abb. 10), dass häusliches Handwerk auch im Freien stattgefunden haben dürfte.

\section{Diskussion der Ergebnisse im lokalen und regionalen Kontext}

Das hiermit komplett vorgelegte und funktional-kontextuell diskutierte Inventar aus Haus 7 des Ada Tepe erlaubt einen ersten Einblick in das Alltagsleben der spätbronzezeitlichen Gesellschaft des 15. Jhs. v. Chr. auf dem Berg. Das Ensemble spiegelt eine sehr selektive Auswahl an Gefäßen und Geräten wider, die auf die Funktion eines Speicherraums mit Lebensmittelverarbeitung (z. B. Getreidemahlen) bzw. auch häuslichem Handwerk schließen lassen. Das Fehlen von Kochgerätschaften und pyrotechnischen Installationen im Gebäudeinneren bekräftigt diese Interpretation. Die insgesamt eher leichte Bauweise lässt uns an einen Schuppen denken, der den Bewohnern des Nordostquartiers als Lager- und Arbeitsstätte gedient haben dürfte. Im Vergleich mit anderen in etwa gleichzeitigen Siedlungen ist der Anteil an Konsumgeschirr in Relation zur Vorratshaltung auffallend gering. Der Anteil an Tassen, Schalen und Schüsseln scheint in den Siedlungsbefunden von Dragojna, Kuš Kaja und Koprivlen höher zu sein - auch wenn hier keine statistischen Analysen vorliegen - wo zudem auch zusätzlich das Kochgeschirr belegt ist. ${ }^{44}$

44 Dragojna: Bozhinova, Jung, Mommsen 2013, 53. - Kuš Kaja: Popov 2009. - Popov 2016, 430. - Koprivlen: Alexandrov 2002, 70-75. 
Ein ähnliches Bild kennen wir auch aus den Tellsiedlungen der Nordägäis, wo wir in den als Wohnräumen genutzten spätbronzezeitlichen Hausinventaren einen deutlich höheren Anteil von Tafelgeschirr und Kochgeräten kennen. So besteht das Ensemble aus Kastanas Schicht 17 aus 86 Konsumgefäßen (45 Schalen, 39 Schüsseln, 2 Tassen), maximal 84 Lagerungs- oder Ausgußgefäßen (6 Amphoren, 78 Töpfe), 15 Pithoi zur Vorratshaltung und 16 Pyraunoi zum Kochen. ${ }^{45}$ Stelios Andreou und Kiki Psaraki haben die soziokulturelle Bedeutung von Ess- und Trinkritualen für die Toumba Thessaloniki herausgearbeitet, wofür gerade das Tafelgeschirr eine wesentliche Rolle spielt. ${ }^{46}$ Eine funktionale Unterscheidung von Lager- und Wohnräumen ist in Siedlungen durchaus nicht ungewöhnlich, wie die etwas jüngeren Speicher aus Assiros zeigen, wo sie auch als Indiz für organisierte Verteilungssysteme innerhalb der spätbronzezeitlichen Gesellschaften gedeutet werden. ${ }^{47}$ Eine umfassendere Studie von Despina Margomenou veranschaulicht, dass auch in Kastanas und der Thessaloniki Toumba verschiedene Formen der Magazinierung in Kontexten mit häuslichem Handwerk und Konsum zu beobachten sind. ${ }^{48}$

Auch auf dem Ada Tepe dürfte sich die Tendenz zu einer funktionalen Differenzierung in Wohnbauten und Speicher abzeichnen, wie die ersten statistischen Auswertungen der Häuser 5 und 9 durch Laura Burkhardt vermuten lassen. ${ }^{49}$ Zumindest Haus 7 lässt sich aufgrund der hier diskutierten Ergebnisse als Speicher und mögliche häusliche Werkstätte interpretieren. Inwieweit diese funktional differenzierte Struktur auch auf andere bulgarische Siedlungen in der Spätbronzezeit zutrifft, ist mangels statistischer Auswertungen und fehlender Gesamtvorlagen derzeit nicht zu beurteilen. Die hier indirekt erschlossene Vorratshaltung von Lebensmitteln in mindestens einem separaten Schuppen des Nordostquartiers legt eine organisierte Versorgung der Bergwerksgesellschaft nahe. ${ }^{50} \mathrm{Da}$ Ackerbau auf dem Berg selbst kaum anzunehmen ist, mussten die Grundversorgungsmittel aus dem Tal nach oben gebracht werden. Die organisierte systematische Lagerung in mindestens einem

45 Die hier nur summarisch aufgelistete Nutzung beinhaltet keine Kantharoi (10 Exemplare in Schicht 17) aufgrund ihrer nicht eindeutigen Zuweisungsmöglichkeit. - HochstetTer 1984, 203-207.

46 Andreou, Psaraki 2007.

47 Jones et al. 1986. - Zur Datierung des Speichers (Phase 9) in SH IIIA2/B siehe Wardle, Wardle 2007, bes. 459-463 und Taf. 4-5. 48 Margomenou 2008.

49 Posterpräsentation im Rahmen einer Konferenz vom 8. bis 10.6.2017 an der Österreichischen Akademie der Wissenschaften in Wien.

50 Siehe dazu auch Popov et al. 2017 und Jung et al. 2017 (beide in diesem Band). eigenen Speicher spricht für eine längerfristige, auf jeden Fall mehrmonatige oder saisonal durchgehende Versorgung der Bergwerksleute. Die (wenn auch geringere) Verwendung von regional-typischem Tafelgeschirr zeigt, dass hier nicht nur eine minimale Versorgung vor Ort stattfand, sondern auch durchaus übliches Ess- und Trinkgeschirr von den Produzenten des Goldes verwendet wurde. Wir erahnen damit eine Gesellschaft von Bergwerksleuten, die in lokaler und regionaler Tradition verwurzelt sind (Abb. 4). Die oben diskutierten Vergleiche der einzelnen Gefäßgattungen und ihre typologische wie auch technologische Varianz veranschaulichen einerseits die kulturelle Einbettung in die Keramiktradition der spätbronzezeitlichen Rhodopen als auch eine weitere überregionale Kontextualisierung mit den Nachbarregionen andererseits (Plovdiv-Čerkovna-Gruppe und Makedonien). Inwieweit sich auf dem Ada Tepe eine eigene lokale Keramikproduktion definieren lässt (die möglicherweise im Tal zu verorten ist), wird erst die detaillierte Auswertung des gesamten Materials zeigen, das derzeit durch Laura Burkhardt und Krasimir Nikov in Bearbeitung ist. Die Analyse des Ensembles aus Haus 7 zeigt zumindest, dass die Goldproduzenten des Ada Tepe im 15. Jh. v. Chr. lokale bis regionale Traditionen in der Art ihrer Vorratshaltung sowie den Ess- und Trinksitten pflegten. Eine entsprechende kulturelle Einbettung der Gold-abbauenden Gesellschaft auf dem Berg ist daher anzunehmen.

\section{Katalog (Catalogue)}

\subsection{Pottery}

\section{Unit 1}

Amphora

$\begin{array}{ll}\begin{array}{ll}\text { Object ID: AT13/1/1/3 } \\ \text { Shape: }\end{array} & \text { amphora } \\ \text { Type: } & \text { conical neck - bent outwards } \\ \text { Variant: } & \text { regular rounded lip } \\ \text { Type code: } & \text { A2A } \\ \text { Preservation: } & \text { secondary burn } \\ \text { Fabric: } & 3 \\ \text { Dimensions (cm): } & \\ & \text { wall thickness: } 0.9 \\ & \text { diam. rim: } 18 \\ & \text { diam. rim, preserv. }(\%): 6\end{array}$

$\begin{array}{ll}\text { Object ID: AT13/1/1/1 } & \text { Tafel 1/1 } \\ \text { Shape: } & \text { amphora } \\ \text { Type: } & \text { cylindrical neck - conical } \\ \text { Variant: } & \text { slightly thickened lip } \\ \text { Type code: } & \text { A1B } \\ \text { Preservation: } & \text { abraded } \\ \text { Fabric: } & 6 \\ \text { Description: } & \text { surface partly preserved, medium burnished }\end{array}$



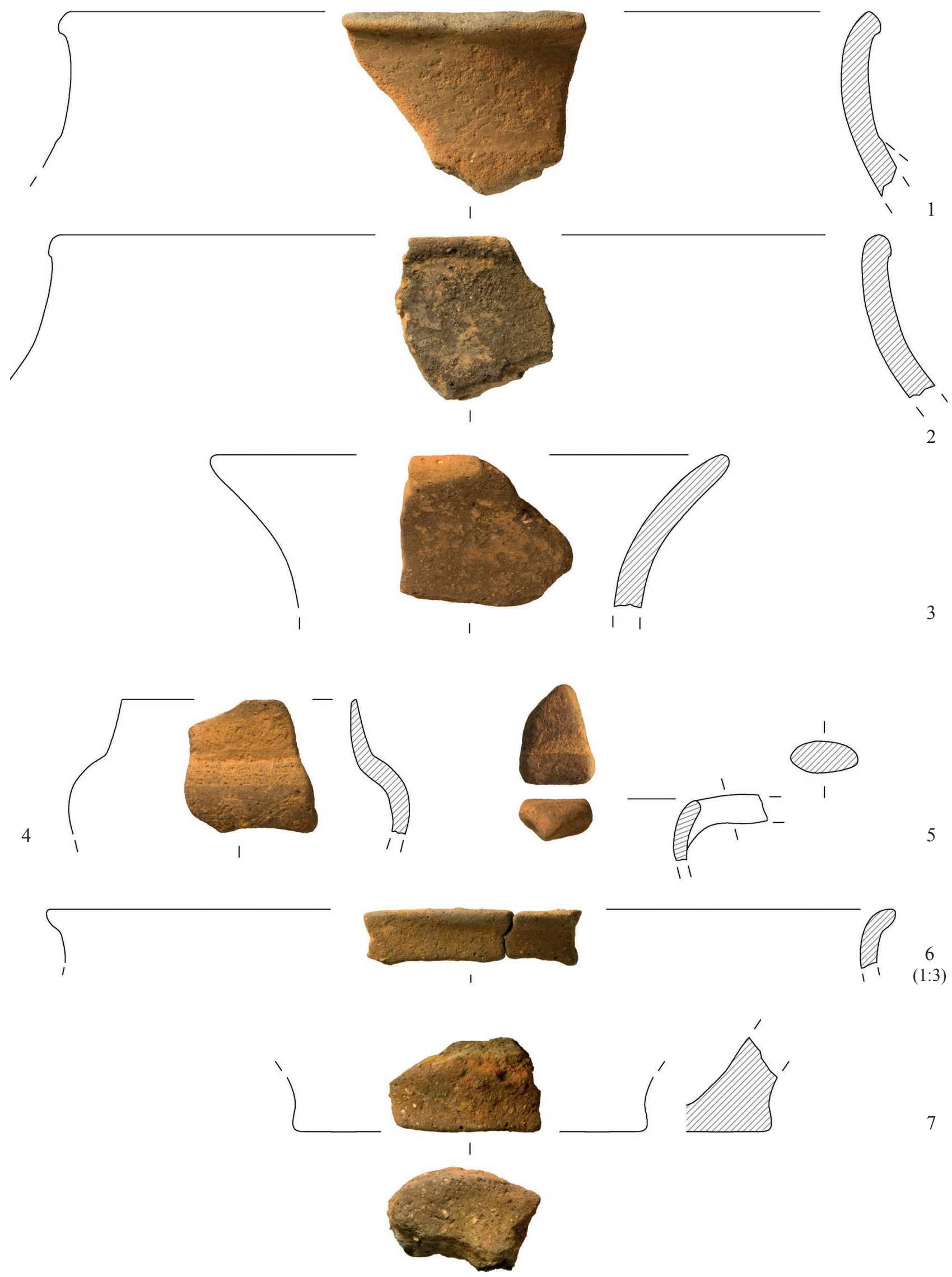

Tafel 1. Ada Tepe, Nordostquartier, Haus 7. Keramik. - 1-5, 7. SE 01 (1:2). - 6. SE 01 (1:3) (Zeichnungen: S. Eder, B. Horejs, St. Horvath, D. Kolic, S. Molla-Djafari, M. Röcklinger; Fotos: Th. Urban). 
Dimensions (cm):

wall thickness: 0.9

diam. rim: 28

diam. rim, preserv. (\%): 9.4

\section{Jar}

Object ID: AT13/1/1/2 Tafel 1/2

Shape: jar

Type: narrow mouthed - concave

Variant: slightly thickened lip

Type code: J1B

Preservation: secondary burn

Fabric: 2

Dimensions $(\mathrm{cm})$ :

wall thickness: 0.9

diam. rim: 28

diam. rim, preserv. (\%): 4

Object ID: AT13/1/1/4

Shape: jar

Type: narrow mouthed - conical

Variant: regular rounded lip

Type code: J2A

Preservation: abraded

Fabric: 4

Application: PC1A

Description: finger impressions

Dimensions $(\mathrm{cm})$ :

wall thickness: 1

diam. rim: 22

diam. rim, preserv. (\%): 9

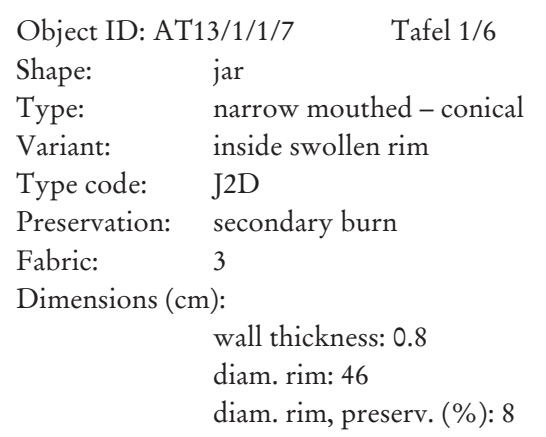

\section{Kantharos}

Object ID: AT13/1/1/5 Tafel 1/4

Shape: kantharos

Type: $\quad$ cylindrical neck - globular

Variant: thinned lip

Type code: K1C

Preservation: abraded

Fabric: $\quad 10$

Application: DF9

Description: double spiral with rays on both ends of the spiral, five horizontal lines, as bundle, on the shoulder

Dimensions (cm):

wall thickness: 0.6

diam. rim: 8

diam. rim, preserv. (\%): 3

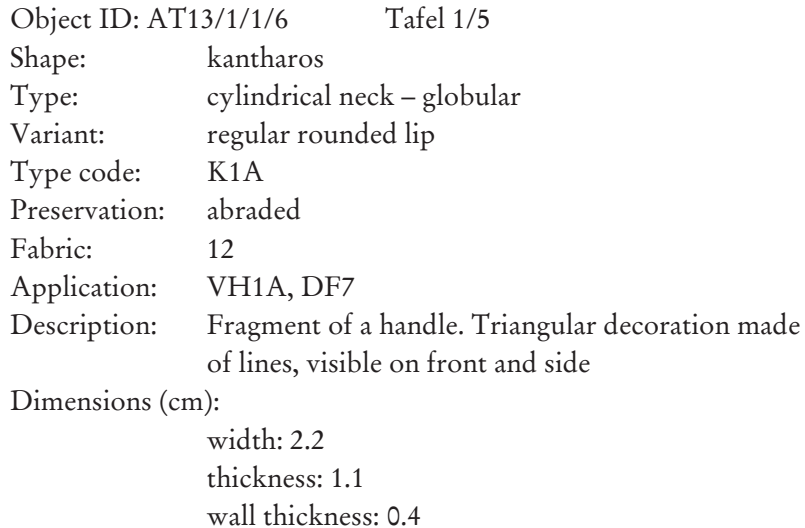

Base

Object ID: AT13/1/1/8 Tafel 1/7

Shape: base

Type: $\quad$ stepped base

Variant: curved

Type code: B1A

Preservation: secondary burn

Fabric: $\quad 4$

Dimensions (cm):

wall thickness: 1.4

diam. base: 12

diam. base, preserv. (\%): 12

Object ID: AT13/1/1/9

Shape: base

Type code: $\quad$ BO

Preservation: secondary burn

Fabric: 1

Dimensions $(\mathrm{cm})$ :

wall thickness: 1.5

\section{Vertical handle}

Object ID: AT13/1/1/13 Tafel 2/2

Shape: vertical handle

Type: oval cross section

Variant: undecorated

Type code: VH1B

Preservation: abraded

Fabric: 2

Dimensions (cm):

width: 3.7

thickness: 3.3

Object ID: AT13/1/1/10 Tafel 2/1

Shape: vertical handle

Type: oval cross section

Variant: undecorated

Type code: VH1B

Preservation: abraded

Fabric: $\quad 1$

Dimensions (cm):

width: 5.2

thickness: 2.1

wall thickness: 0.7 

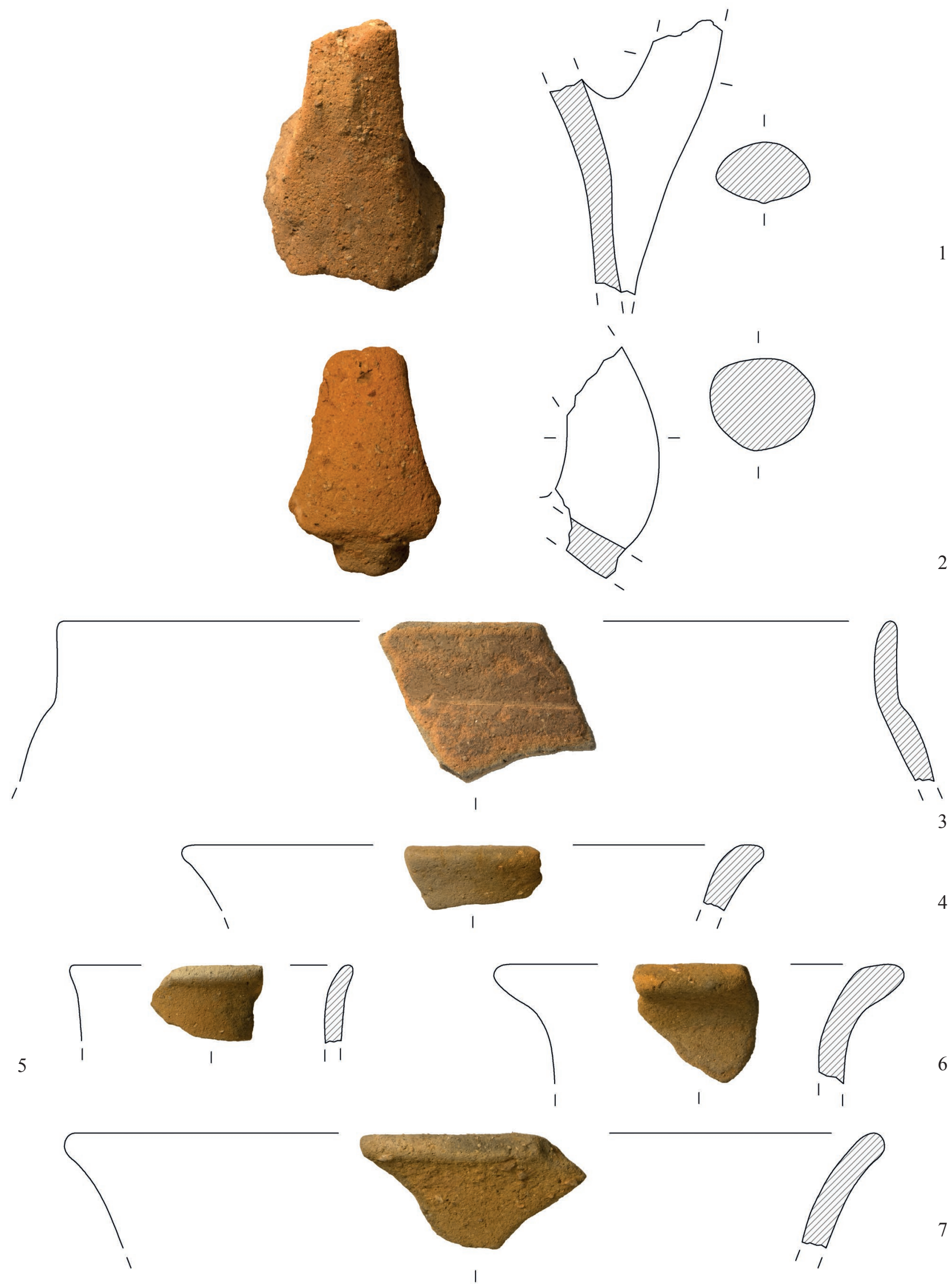

6

Tafel 2. Ada Tepe, Nordostquartier, Haus 7. Keramik. - 1-2. SE 01 (1:2). - 3-7. SE 02 (1:2) (Zeichnungen: S. Eder, B. Horejs, St. Horvath, D. Kolic, S. Molla-Djafari, M. Röcklinger; Fotos: Th. Urban). 
Object ID: AT13/1/1/11

Shape: vertical handle

Type: oval cross section

Variant: undecorated

Type code: VH1B

Preservation: abraded

Fabric: 2

Dimensions (cm):

width: 3.84

thickness: 3.2

Object ID: AT13/1/1/12

Shape: vertical handle

Type: oval cross section

Variant: undecorated

Type code: VH1B

Preservation: abraded

Fabric: 3

Dimensions $(\mathrm{cm})$ :

width: 4

thickness: 3.1

Object ID: AT13/1/1/15

Shape: vertical handle

Type: oval cross section

Variant: undecorated

Type code: VH1B

Preservation: abraded

Fabric: 1

Dimensions $(\mathrm{cm})$ :

width: 3.2

thickness: 2.8

Object ID: AT13/1/1/16

Shape: vertical handle

Type: oval cross section

Variant: undecorated

Type code: VH1B

Preservation: abraded

Fabric: 1

Dimensions $(\mathrm{cm})$ :

width: 3

thickness: 2.5

Object ID: AT13/1/1/18

Shape: vertical handle

Type: oval cross section

Variant: undecorated

Type code: VH1B

Preservation: abraded

Fabric: 6

Dimensions $(\mathrm{cm})$ :

width: 4

thickness: 2.5

Object ID: AT13/1/1/19

Shape: vertical handle

Type: rhombic cross section

Variant: undecorated

Type code: VH2A

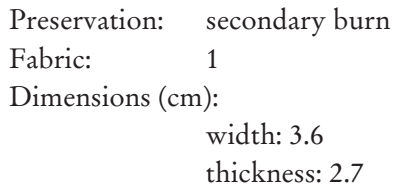

Object ID: AT13/1/1/21

Shape: vertical handle

Type: oval cross section

Variant: undecorated

Type code: VH1B

Preservation: abraded

Fabric: $\quad 10$

Dimensions (cm):

width: 2.3

thickness: 1.1

wall thickness: 0.7

Horizontal handle

Object ID: AT13/1/1/14

Shape: horizontal handle

Type: oval cross section

Variant: undecorated

Type code: HH1A

Preservation: abraded

Fabric: $\quad 4$

Dimensions (cm):

width: 3.1

thickness: 3.2

wall thickness: 1.3

Decorated wall fragment

Object ID: AT13/1/1/22

Shape: decorated wall fragment

Type: $\quad$ Furchenstich

Type code: DF10

Preservation: abraded

Fabric: $\quad 5$

Application: DF10

Description: two to three cut-out triangles along the bundle of four lines

Dimensions (cm):

wall thickness: 0.7

Unit 2

Amphora

Object ID: AT13/2/1/7

Shape: amphora

Type: cylindrical neck - conical 
Dimensions (cm):

wall thickness: 0.6

diam. rim: 10

diam. rim, preserv. (\%): 9

Open vessel

Object ID: AT13/2/1/11

Shape: open vessel

Variant: thinned lip

Type code: OV1C

Preservation: abraded

Fabric: 4

Dimensions (cm):

$$
\begin{aligned}
& \text { wall thickness: } 0.7 \\
& \text { diam. rim: } 20 \\
& \text { diam. rim, preserv. (\%): } 6
\end{aligned}
$$

\section{Rim}

Object ID: AT13/2/1/5

Shape: rim

Type: not definable

Variant: squared lip

Type code: ROE

Preservation: abraded

Fabric: 1

\section{Base}

Object ID: AT13/2/1/15 Tafel 3/3

Shape: base

Type: stepped base

Variant: flat

Type code: B1B

Preservation: abraded

Fabric: 3

Dimensions $(\mathrm{cm})$ :

wall thickness: 1.5

diam. base: 12

diam. base, preserv. (\%): 26

Object ID: AT13/2/1/14

Shape: base

Type: flat base

Variant: sharp transition

Type code: B2A

Preservation: abraded

Fabric: 4

Dimensions $(\mathrm{cm})$ :

wall thickness: 0.7

diam. base: 5

diam. base, preserv. (\%): 12

Object ID: AT13/2/1/19

Shape: base

Type: slightly stepped

Type code: $\quad$ B30

Preservation: abraded

Fabric: 1

Dimensions (cm):

wall thickness: 1.5
Object ID: AT13/2/1/16

Shape: base

Type: stepped base

Variant: flat

Type code: B1B

Preservation: abraded

Fabric: 1

Dimensions (cm):

diam. base: 10

diam. base, preserv. (\%): 19

Object ID: AT13/2/1/17 Tafel 3/4

Shape: base

Type: slightly stepped

Variant: curved

Type code: B4A

Preservation: abraded

Fabric: 11

Dimensions $(\mathrm{cm})$ :

wall thickness: 0.7

diam. base: 9

diam. base, preserv. (\%): 18
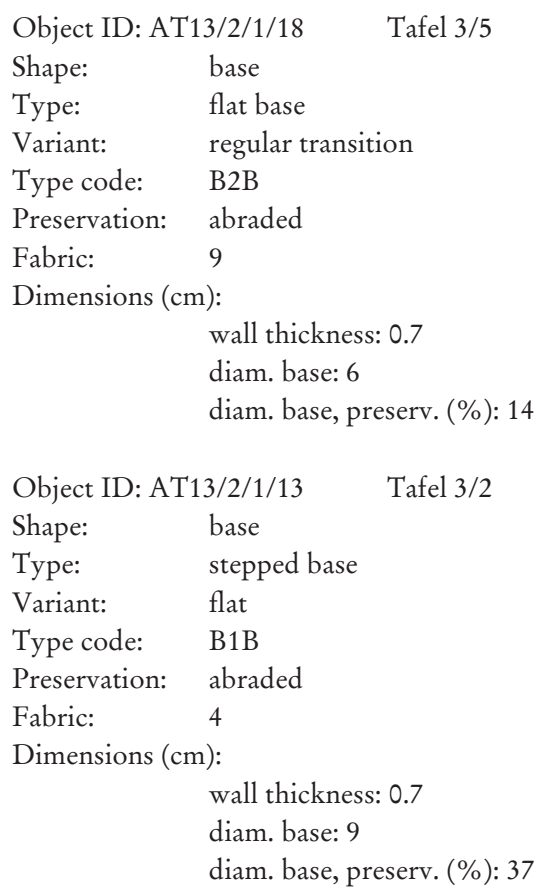

\section{Vertical handle}

Object ID: AT13/2/1/21

Shape: vertical handle

Type: oval cross section

Variant: undecorated

Type code: VH1B

Preservation: secondary burn

Fabric: 1

Dimensions $(\mathrm{cm})$ :

width: 3.1

thickness: 2.4 

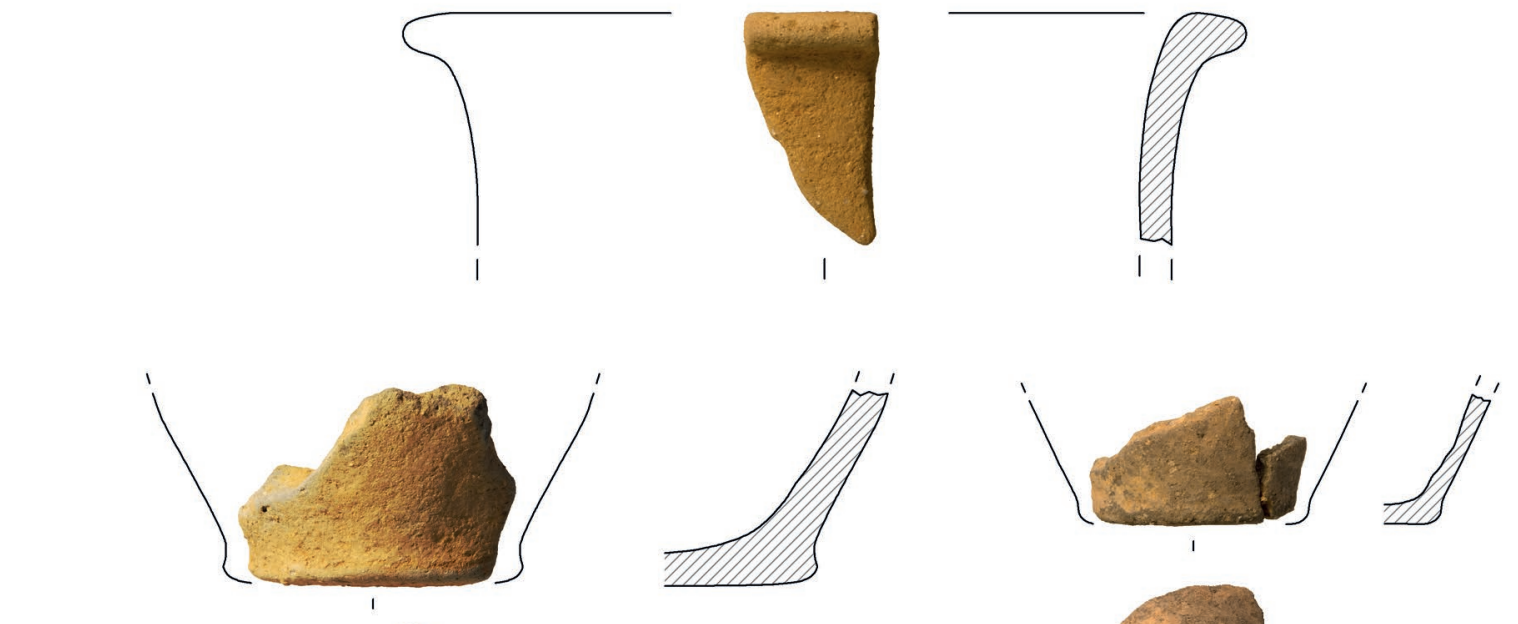

3
$(1: 4)$
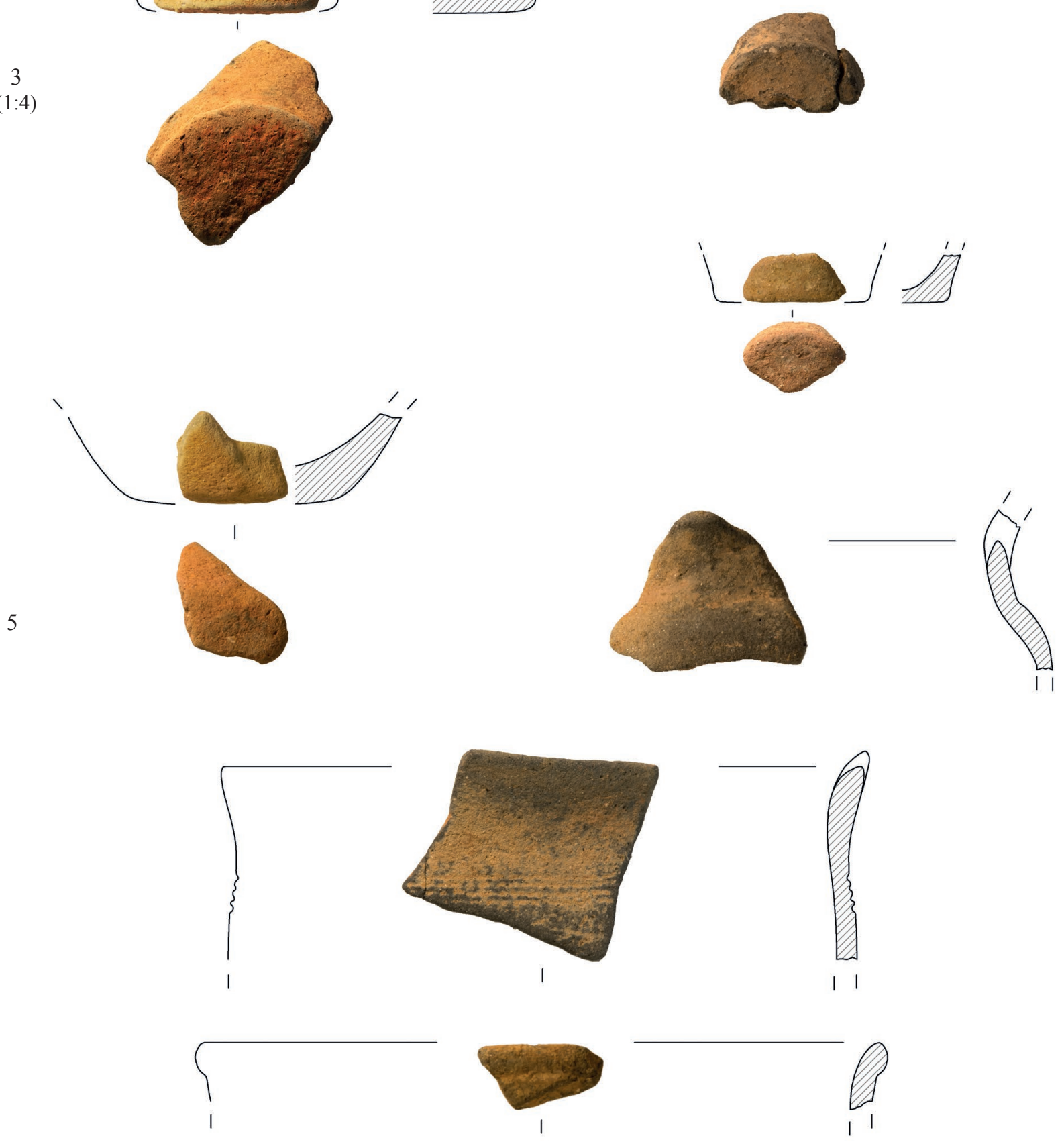

Tafel 3. Ada Tepe, Nordostquartier, Haus 7. Keramik. - 1, 5. SE 02 (1:2). - 2-4. SE 02 (1:4). - 6-8. SE 03 (1:2) (Zeichnungen: S. Eder, B. Horejs, St. Horvath, D. Kolic, S. Molla-Djafari, M. Röcklinger; Fotos: Th. Urban). 
Object ID: AT13/2/1/24

Shape: vertical handle

Type: oval cross section

Variant: undecorated

Type code: VH1B

Preservation: abraded

Fabric: 5

Dimensions $(\mathrm{cm})$ :

width: 2.9

thickness: 1.6

Object ID: AT13/2/1/22

Shape: vertical handle

Type: oval cross section

Variant: undecorated

Type code: VH1B

Preservation: abraded

Fabric: $\quad 5$

Dimensions $(\mathrm{cm})$ :

width: 3.5

thickness: 2.6

\section{Decorated wall fragment}

Object ID: AT13/2/1/29

Shape: decorated wall fragment

Type: grooved

Variant: grooved

Type code: DF3

Preservation: abraded

Fabric: $\quad 5$

Description: two bundles of lines (more than five lines) running slantwise, starting at a horizontal bundle of lines (three lines)

Dimensions $(\mathrm{cm})$ :

diam. shoulder: 12

diam. shoulder, preserv. (\%): 9

wall thickness: 0.6

Object ID: AT13/2/1/28

Shape: decorated wall fragment

Type: incised

Type code: DF9

Preservation: abraded

Fabric: $\quad 10$

Description: angular and slantwise bundles of lines (three to four lines)

Dimensions (cm): wall thickness: 0.8

Object ID: AT13/2/1/27

Shape: decorated wall fragment

Type: incised

Variant: Furchenstich

Type code: DF9

Preservation: abraded

Fabric: 3

Description: two to three badly preserved lines

Dimensions (cm):

wall thickness: 0.8
Unit 3

Amphora

Object ID: AT13/3/1/5 Tafel 4/1

Shape: amphora

Type: $\quad$ conical neck - bent outwards

Variant: thinned lip

Type code: A2C

Preservation: abraded

Fabric: $\quad 4$

Dimensions $(\mathrm{cm})$ :

wall thickness: 0.5

diam. rim: 25

diam. rim, preserv. (\%): 1

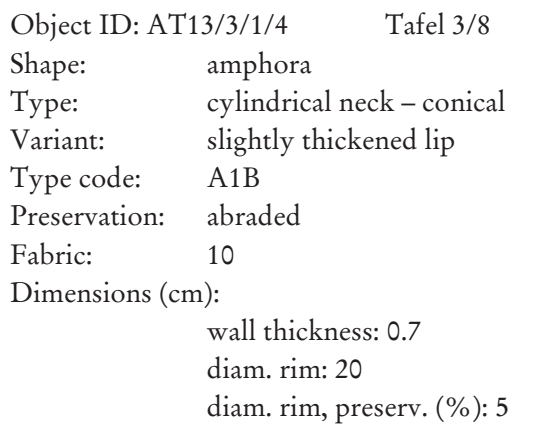

Jar

Object ID: AT13/3/1/13

Shape: jar

Type: $\quad$ cylindrical neck - egg shaped

Variant: slightly thickened lip

Type code: J3B

Preservation: abraded

Fabric: $\quad 11$

Application: PC1A, PC2A

Dimensions $(\mathrm{cm})$

wall thickness: 1.2

diam. rim: 58

diam. rim, preserv. (\%): 2.9

Kantharos

Object ID: AT13/3/1/6 Tafel 3/6

Shape: kantharos

Type: cylindrical neck - globular

Variant: regular rounded lip

Type code: K1A

Preservation: abraded

Fabric: $\quad 10$

Application: $\quad \mathrm{VH}$

Dimensions (cm):

wall thickness: 0.6

Deep bowl

Object ID: AT13/3/1/1

Shape: deep bowl

Type: funnel shaped neck

Variant: bevelled rim, regular rounded lip

Type code: DB1F 

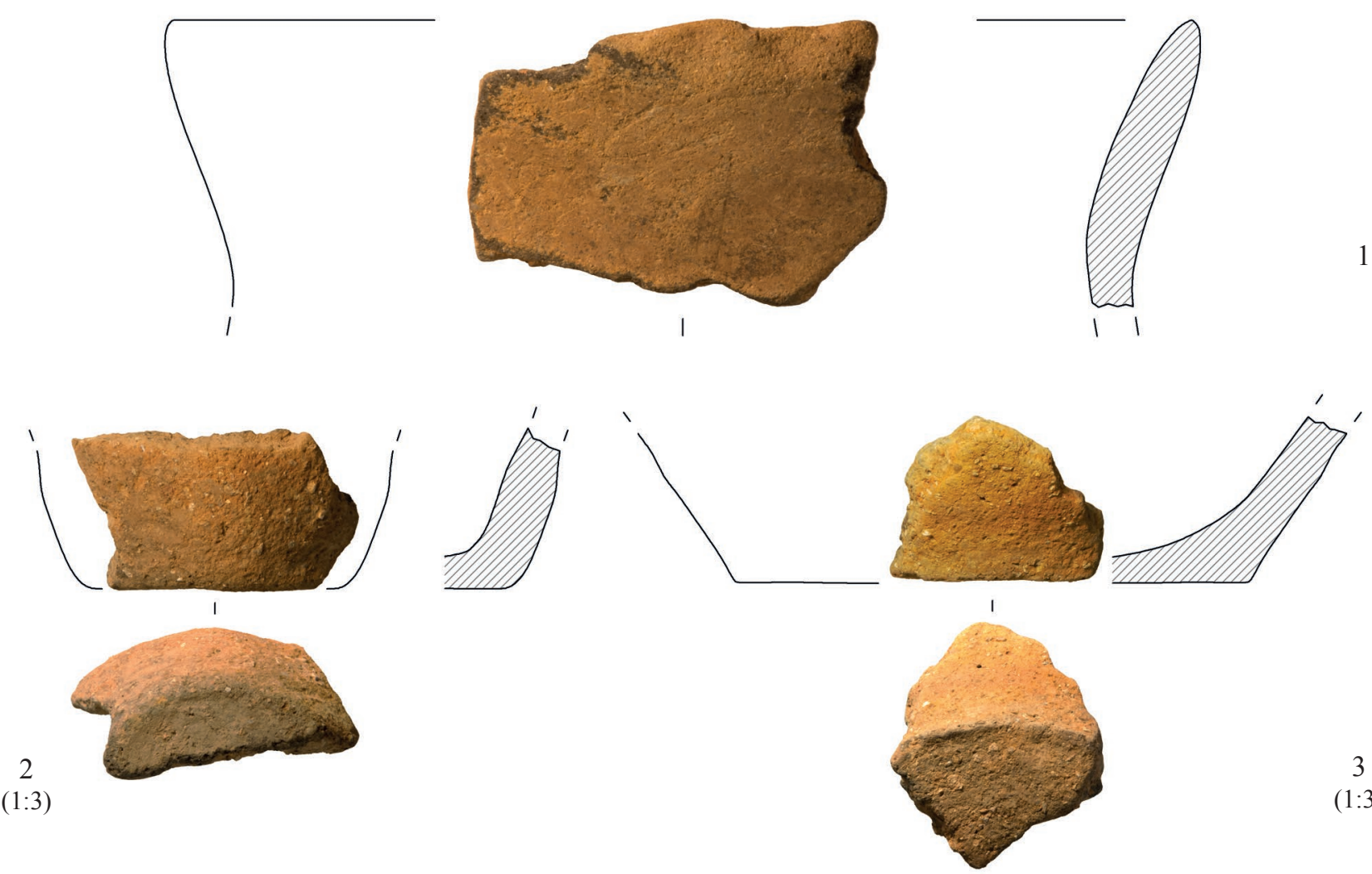

3
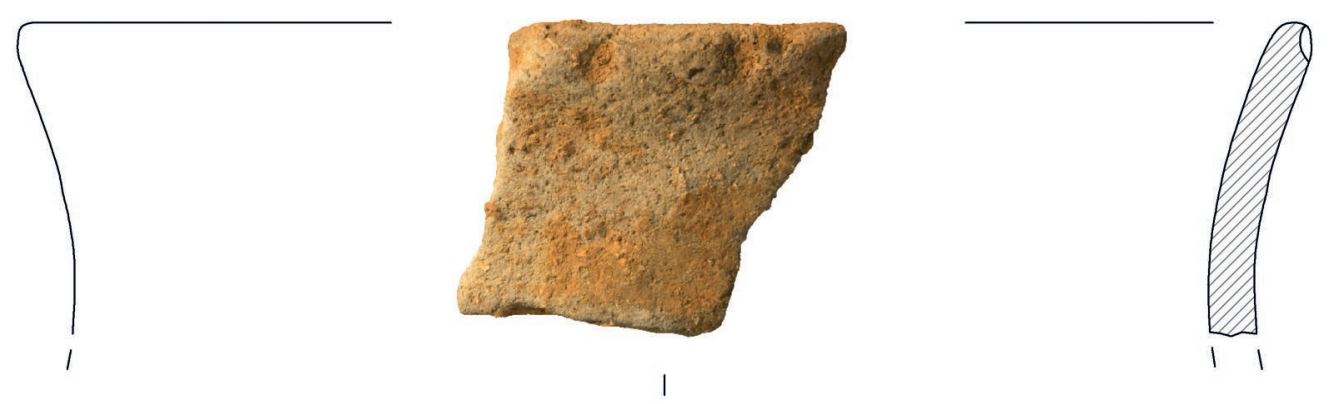

4
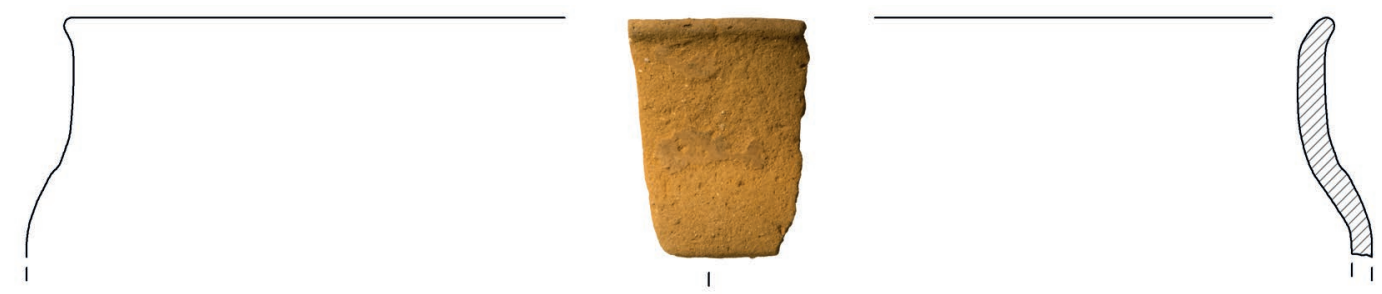

6
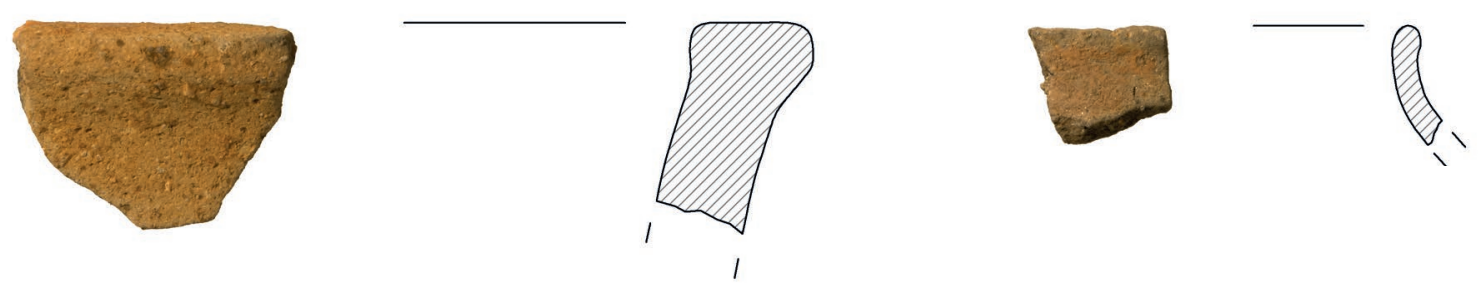

Tafel 4. Ada Tepe, Nordostquartier, Haus 7. Keramik. - 1. SE 03 (1:2). - 2-3. SE 03 (1:3). - 4, 6-7. SE 04 (1:2). - 5. SE 04 (1:3) (Zeichnungen: S. Eder, B. Horejs, St. Horvath, D. Kolic, S. Molla-Djafari, M. Röcklinger; Fotos: Th. Urban). 
Preservation: abraded

Fabric: 6

Dimensions (cm):

wall thickness: 0.8

diam. rim: 32

diam. rim, preserv. (\%): 19

\section{Open vessel}

\begin{tabular}{|c|c|}
\hline Object ID: AT & Tafel 3/7 \\
\hline Shape: & open vessel \\
\hline Type: & sloping rim \\
\hline Variant: & inside swollen rim \\
\hline Type code: & OV1D \\
\hline Preservation: & abraded \\
\hline Fabric: & 10 \\
\hline Application: & DF9 \\
\hline Description: & $\begin{array}{l}\text { diameter took at the neck; bundle of lines } \\
\text { (four to five) on neck; cup? }\end{array}$ \\
\hline
\end{tabular}

Dimensions (cm):

$$
\begin{aligned}
& \text { wall thickness: } 0.6 \\
& \text { diam. rim: } 18.5 \\
& \text { diam. rim, preserv. (\%): } 9
\end{aligned}
$$

\section{Closed vessel}

Object ID: AT13/3/1/17

Shape: closed vessel

Variant: $\quad$ regular rounded lip

Type code: CvA

Preservation: abraded

Fabric: $\quad 6$

Dimensions $(\mathrm{cm})$ :

wall thickness: 0.6

\section{Base}

Object ID: AT13/3/1/8 Tafel 4/3

Shape: base

Type: flat base

Variant: sharp transition

Type code: B2A

Preservation: abraded

Fabric: 1

Dimensions (cm):

wall thickness: 1

diam. base: 18

diam. base, preserv. (\%): 3

$\begin{array}{ll}\text { Object ID: AT13/3/1/7 } & \text { Tafel 4/2 } \\ \text { Shape: } & \text { base } \\ \text { Type: } & \text { curved } \\ \text { Variant: } & \text { regular transition } \\ \text { Type code: } & \text { B3A } \\ \text { Preservation: } & \text { abraded } \\ \text { Fabric: } & 1 \\ \text { Dimensions (cm): } & \\ & \text { wall thickness: } 1 \\ & \text { diam. base: } 9 \\ & \text { diam. base, preserv. }(\%): 26\end{array}$

Vertical handle

Object ID: AT13/3/1/9

Shape: vertical handle

Type: oval cross section

Variant: undecorated

Type code: VH1B

Preservation: abraded

Fabric: 1

Dimensions $(\mathrm{cm})$ :

width: 4

thickness: 1.9

wall thickness: 0.8

Object ID: AT13/3/1/12

Shape: $\quad$ vertical handle

Type: rhombic cross section

Variant: undecorated

Type code: $\quad$ VH2A

Preservation: abraded

Fabric: 1

Dimensions $(\mathrm{cm})$ :

width: 3.5

thickness: 3.3

Object ID: AT13/3/1/10

Shape: vertical handle

Type: oval cross section

Variant: undecorated

Type code: VH1B

Preservation: abraded

Fabric: $\quad 3$

Dimensions $(\mathrm{cm})$ :

width: 1.7

thickness: 2.5

wall thickness: 0.5

Object ID: AT13/3/1/11

Shape: vertical handle

Type: oval cross section

Variant: undecorated

Type code: VH1B

Preservation: abraded

Fabric: $\quad 3$

Dimensions (cm):

width: 2.7

thickness: 1.8

wall thickness: 0.7

Decorated wall fragment

Object ID: AT13/3/1/15

Shape: decorated wall fragment

Type: Furchenstich

Type code: DF9

Preservation: abraded

Fabric: $\quad 10$

Description: possibly rhombic motive; bundle of lines running slantwise, meeting at one point (forming an angle); lines partly connected to each other by hatchings 
Dimensions (cm): wall thickness: 0.7

\section{Unit 4 \\ Amphora \\ Object ID: AT13/4/1/12 Tafel 5/3 \\ Shape: amphora \\ Type: $\quad$ cylindrical neck - conical \\ Variant: $\quad$ regular rounded lip \\ Type code: A2A \\ Preservation: secondary burn \\ Fabric: 6 \\ Dimensions (cm):

wall thickness: 0.8
diam. rim: 16
diam. rim, preserv. (\%): 7

Object ID: AT13/4/1/9

Shape: amphora

Type: not definable

Variant: bevelled rim, regular rounded lip

Type code: AOF

Preservation: abraded

Fabric: 6

Dimensions (cm):

wall thickness: 0.8

Object ID: AT13/4/1/16

Shape: amphora

Type: $\quad$ cylindrical neck - conical

Variant: inside swollen rim

Type code: A1D

Preservation: abraded

Fabric: 5

Dimensions (cm):

wall thickness: 0.9

Object ID: AT13/4/1/13

Shape: amphora

Type: $\quad$ cylindrical neck - conical

Variant: regular rounded lip

Type code: A1A

Preservation: abraded

Fabric: 6

Dimensions ( $\mathrm{cm}$ ):

wall thickness: 0.7

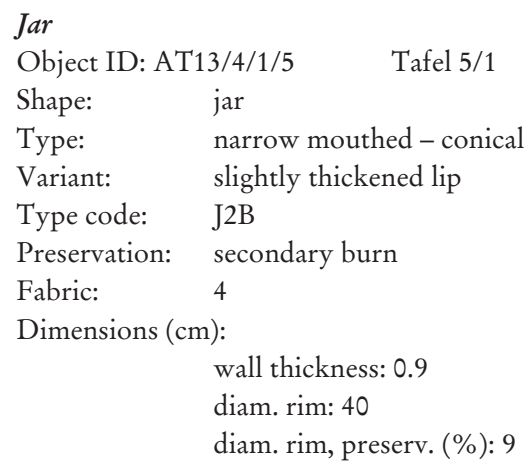

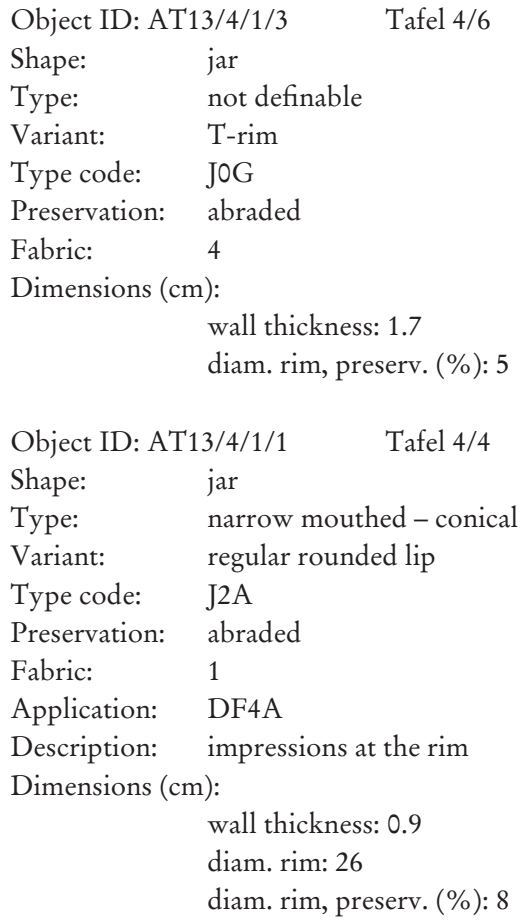

Kantharos

Object ID: AT13/4/1/8 Tafel 4/7

Shape: kantharos

Type: not definable

Variant: $\quad$ regular rounded lip

Type code: KOA

Preservation: abraded

Fabric: $\quad 10$

Dimensions (cm):

wall thickness: 0.5

Object ID: AT13/4/1/15

Shape: kantharos

Type: not definable

Variant: regular rounded lip

Type code: KOA

Preservation: abraded

Fabric: $\quad 10$

Dimensions $(\mathrm{cm})$ :

$$
\text { wall thickness: } 0.4
$$

\section{Deep bowl}

\begin{tabular}{|c|c|}
\hline \multicolumn{2}{|c|}{ Object ID: AT13/4/1/2 } \\
\hline Shape: & deep bowl \\
\hline Type: & cylindrical neck \\
\hline Variant: & slightly thickened lip \\
\hline Type code: & $\mathrm{DB} 2 \mathrm{~B}$ \\
\hline Preservation: & abraded \\
\hline Fitting with $A^{\prime}$ & $\Gamma 13 / 4 / 1 / 6$ \\
\hline Fabric: & 13 \\
\hline Dimensions ( $\mathrm{c}$ & \\
\hline & wall thickness: 0.6 \\
\hline & diam. rim: 52 \\
\hline & diam. rim, preserv. $(9$ \\
\hline
\end{tabular}


Closed vessel

Object ID: AT13/4/1/30 Tafel 5/2

Shape: Closed vessel

Type: not definable

Type code: CVO

Preservation: abraded

Fabric: 12

Application: DF10

Description: Multiple sun motives, lined with cut-out triangles. Bundle of lines leading to hanging triangles (continuous and dashed lines, one

Dimensions $(\mathrm{cm})$ : short line at right angle to lines); amphora?

diam. Body: 28

wall thickness: 0.7

\section{Base}

Object ID: AT13/4/1/21

Shape: base

Type: flat base

Variant: regular transition

Type code: B2B

Preservation: secondary burn

Fabric: 6

Object ID: AT13/4/1/20

Shape: base

Type: stepped base

Variant: curved

Type code: B1A

Preservation: abraded

Fabric: 5

Dimensions (cm):

wall thickness: 1

diam. base: 12

diam. base, preserv. (\%): 12

Object ID: AT13/4/1/19

Shape: base

Type: stepped base

Variant: curved

Type code: B1A

Preservation: abraded

Fabric: 1

Dimensions $(\mathrm{cm})$ : wall thickness: 1.1

$\begin{array}{ll}\text { Object ID: AT13/4/1/17 } & \text { Tafel 5/5 } \\ \text { Shape: } & \text { base } \\ \text { Type: } & \text { stepped base } \\ \text { Variant: } & \text { curved } \\ \text { Type code: } & \text { B1A } \\ \text { Preservation: } & \text { abraded } \\ \text { Fabric: } & 1 \\ \text { Dimensions (cm): } & \\ & \text { wall thickness: } 1.3 \\ & \text { diam. base: } 12 \\ & \text { diam. base, preserv. (\%): } 18\end{array}$

Object ID: AT13/4/1/18

Shape: base

Type: flat base

Variant: regular transition

Type code: $\quad$ B2B

Preservation: abraded

Fabric: $\quad 12$

Dimensions (cm):

wall thickness: 0.8

Vertical handle

Object ID: AT13/4/1/22

Shape: vertical handle

Type: oval cross section

Variant: undecorated

Type code: VH1B

Preservation: abraded

Fabric: $\quad 7$

Dimensions (cm):

width: 2.5

thickness: 1.8

wall thickness: 0.5

Object ID: AT13/4/1/23

$\begin{array}{ll}\text { Shape: } & \text { vertical handle } \\ \text { Type: } & \text { oval cross section } \\ \text { Variant: } & \text { undecorated } \\ \text { Type code: } & \text { VH1B } \\ \text { Fabric: } & 1 \\ \text { Dimensions (cm): } & \text { width: } 3 \\ & \text { thickness: } 2.5\end{array}$

Object ID: AT13/4/1/24

Shape: vertical handle

Type: rhombic cross section

Variant: undecorated

Type code: VH2A

Fabric: $\quad 5$

Dimensions $(\mathrm{cm})$ :

width: 1.6

thickness: 1.4

Object ID: AT13/4/1/26

Shape: vertical handle

Type: oval cross section

Variant: undecorated

Type code: VH1B

Preservation: abraded

Fabric: $\quad 7$

Dimensions $(\mathrm{cm})$ :

width: 2.6

thickness: 2.4

Object ID: AT13/4/1/27

Shape: vertical handle

Type: oval cross section

Variant: undecorated

Type code: VH1B 

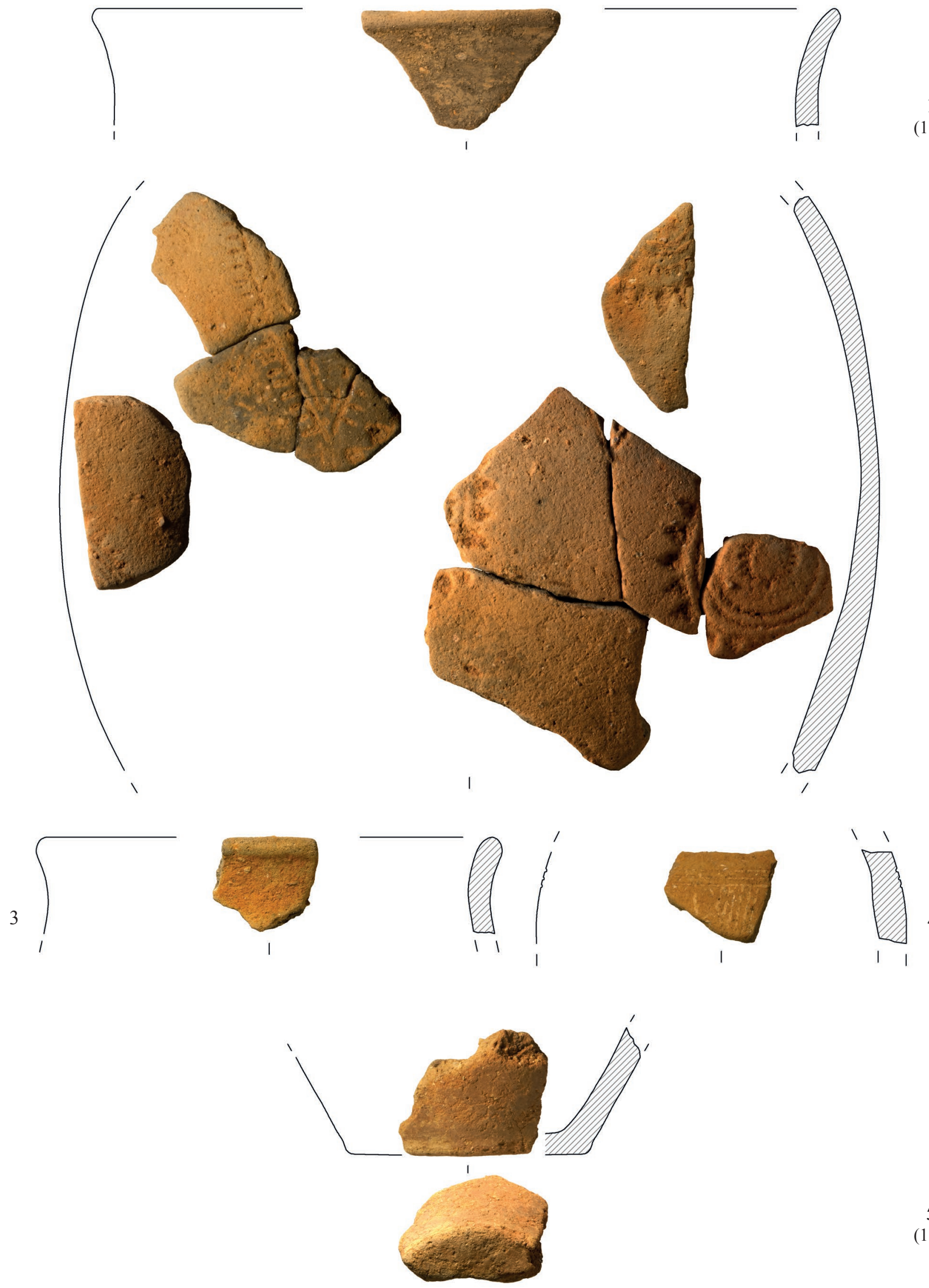

Tafel 5. Ada Tepe, Nordostquartier, Haus 7. Keramik. - 1, 5. SE 04 (1:3). - 2-4. SE 04 (1:2) (Zeichnungen: S. Eder, B. Horejs, St. Horvath, D. Kolic, S. Molla-Djafari, M. Röcklinger; Fotos: Th. Urban). 
Preservation: abraded

Fabric:
Dimensions (cm):

width: 4.3

thickness: 2.5

Object ID: AT13/4/1/29

Shape: vertical handle

Type: oval cross section

Variant: undecorated

Type code: VH1B

Preservation: abraded

Fabric: 7

\section{Decorated wall fragment}

Object ID: AT13/4/1/42

Shape: decorated wall fragment

Type: not definable

Type code: DFO

Preservation: abraded

Fabric: 13

Application: DFO

Description: eventually Furchenstich, bundle of lines Dimensions $(\mathrm{cm})$ :

wall thickness: 0.5

Object ID: AT13/4/1/37 Tafel 5/4

Shape: decorated wall fragment

Type: incrusted

Variant: grooved

Type code: DF9

Preservation: abraded

Fabric: 7

Description: oblique bundle of lines starting at horizontal bundle of line

Dimensions ( $\mathrm{cm})$ :

Diam.: 12

Diam., preserved (\%): 10.5

wall thickness: 1

Object ID: AT13/4/1/34

Shape: decorated wall fragment

Type: Furchenstich

Variant: grooved

Type code: DF9

Preservation: abraded

Fabric: $\quad 10$

Description: oblique bundle of lines starting at horizontal bundle of line

Dimensions (cm):

wall thickness: 0.5

\section{Unit 5}

\section{Amphora}

Object ID: AT13/5/1/3

Tafel 6/3

Shape:

amphora

Type: $\quad$ cylindrical neck - conical

Variant: thinned lip

Type code: A1C

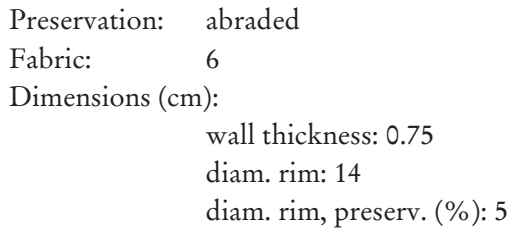

Object ID: AT13/5/1/4

Shape: amphora

Type: not definable

Variant: $\quad$ regular rounded lip

Type code: AOA

Preservation: secondary burn

Fabric: 6

Dimensions $(\mathrm{cm})$ :

wall thickness: 1.2

Jar

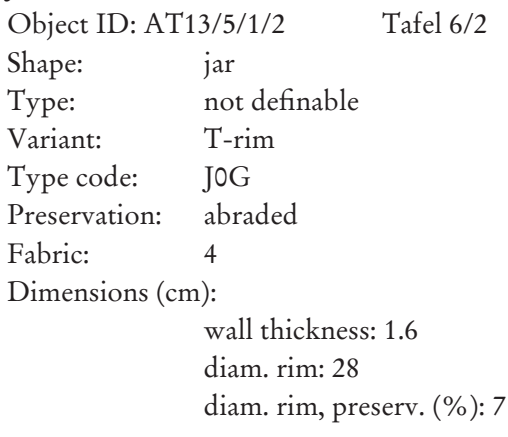

Deep bowl

Object ID: AT13/5/1/1 Tafel 6/1

Shape: deep bowl

Type: not definable

Variant: regular rounded lip

Type code: $\quad$ DBOA

Preservation: abraded

Fabric: 6

Dimensions $(\mathrm{cm})$ :

wall thickness: 0.8

diam. rim: 29

diam. rim, preserv. (\%): 6

Open vessel

Object ID: AT13/5/1/6

Shape: open vessel

Variant: regular rounded lip

Type code: OV1A

Preservation: abraded

Fabric: 1

Dimensions (cm):

wall thickness: 0.9

Closed vessel

Object ID: AT13/5/1/17 Tafel 6/6

Shape: closed vessel

Type: $\quad$ grooved and Furchenstich

Type code: DF6

Preservation: secondary burn 

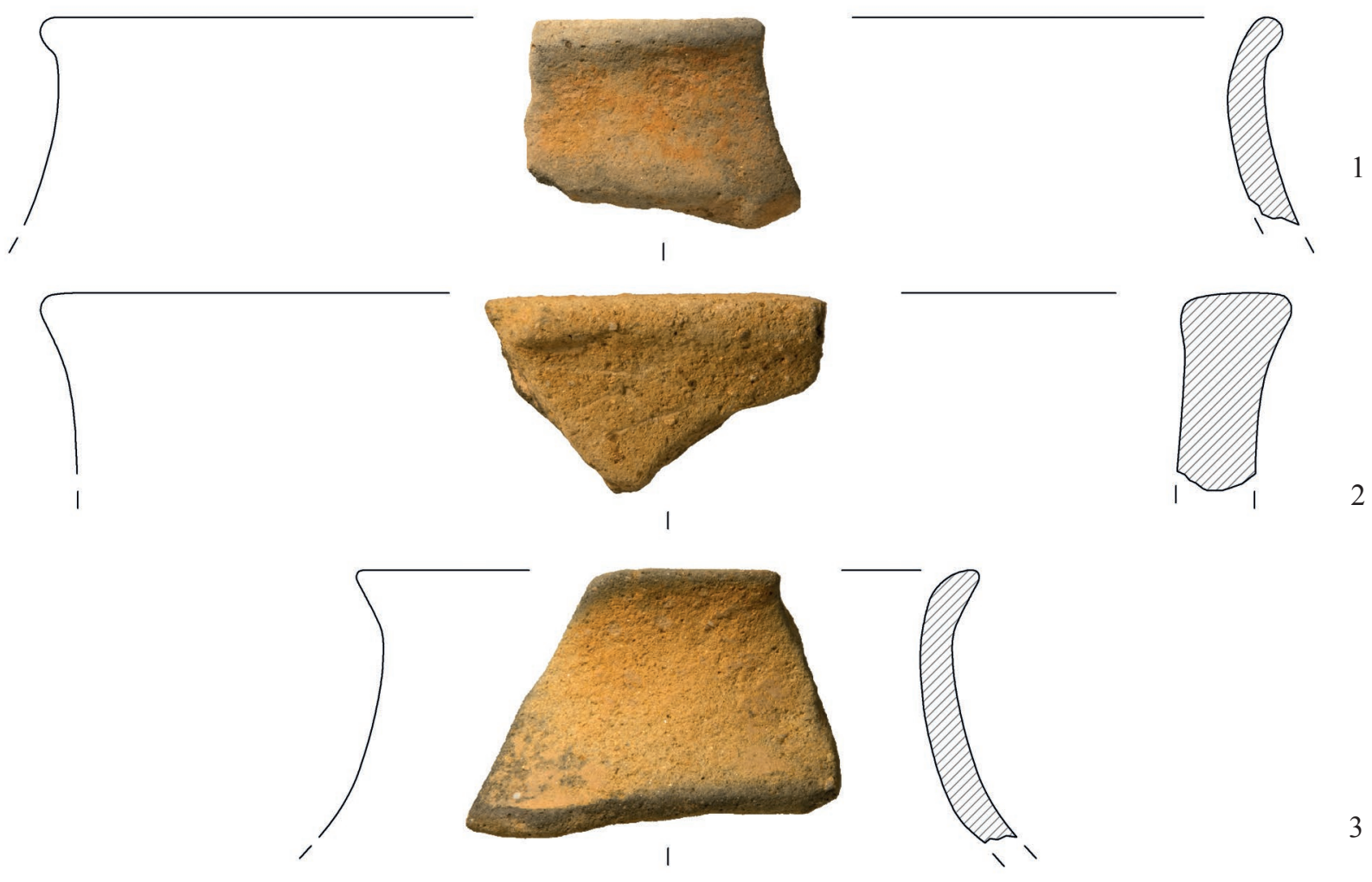

3
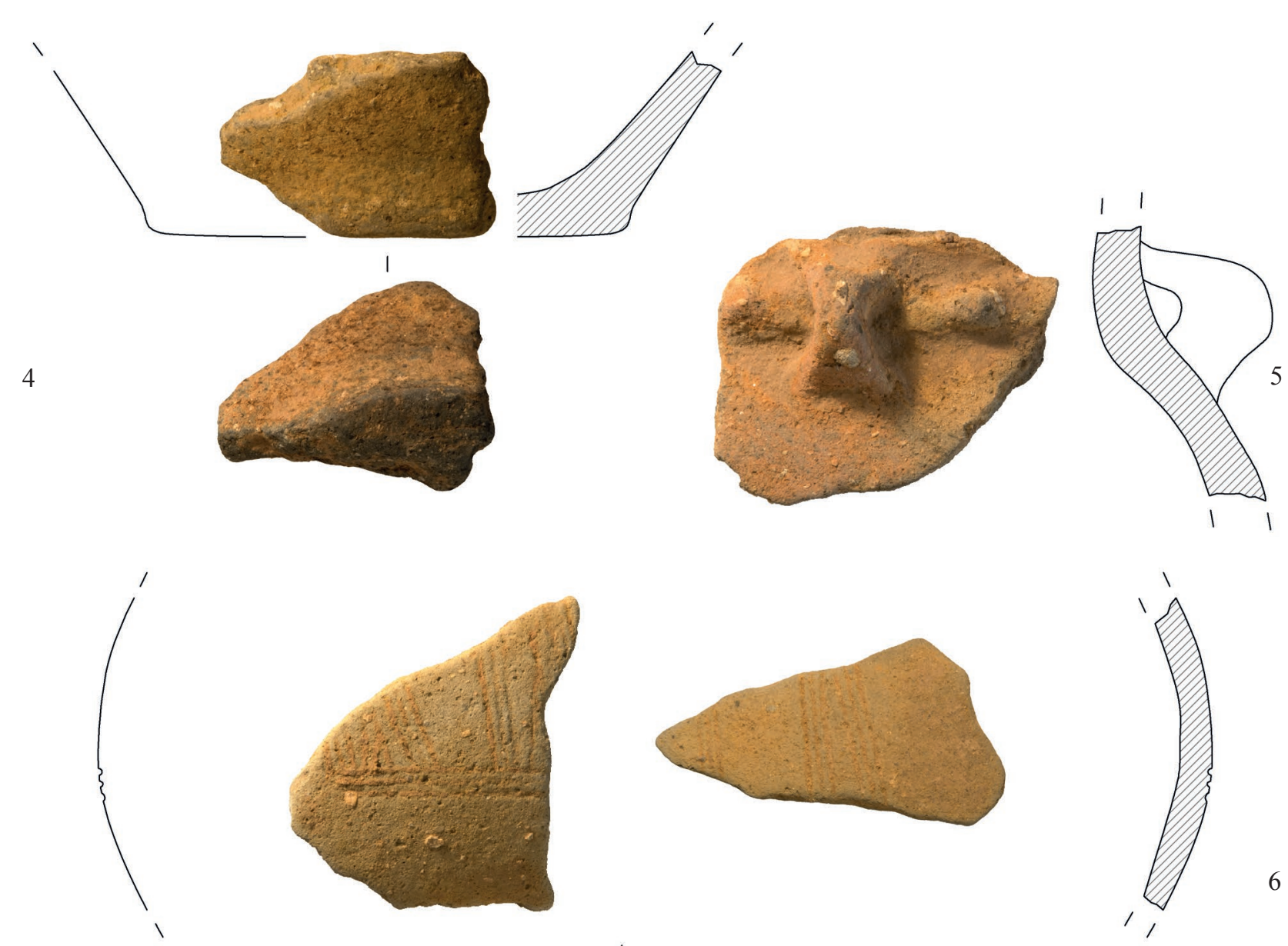

6

Tafel 6. Ada Tepe, Nordostquartier, Haus 7. Keramik. -1-6. SE 05 (1:2) (Zeichnungen: S. Eder, B. Horejs, St. Horvath, D. Kolic, S. Molla-Djafari, M. Röcklinger; Fotos: Th. Urban). 
Fabric:

Description: oblique bundle of lines (meeting in an angle)

Dimensions $(\mathrm{cm})$ starting at horizontal bundle of line

diam. Max: 30

wall thickness: 0.8

Object ID: AT13/5/1/5

Shape: closed vessel

Variant: thinned lip

Type code: $\quad \mathrm{CvC}$

Fabric: $\quad 10$

Dimensions $(\mathrm{cm})$ :

wall thickness: 0.4

\section{Base}

Object ID: AT13/5/1/10

Shape: base

Type: slightly stepped

Variant: curved

Type code: B4A

Preservation: abraded

Fabric: $\quad 10$

Dimensions $(\mathrm{cm})$ :

wall thickness: 0.6

Object ID: AT13/5/1/11

Shape: base

Type: flat base

Variant: regular transition

Type code: B2B

Preservation: secondary burn

Fabric:

10

Dimensions (cm):

wall thickness: 0.4

diam. base: 4

diam. base, preserv. (\%): 100

Object ID: AT13/5/1/9

$\begin{array}{ll}\text { Shape: } & \text { base } \\ \text { Type: } & \text { stepped base } \\ \text { Variant: } & \text { curved } \\ \text { Type code: } & \text { B1A } \\ \text { Preservation: } & \text { abraded } \\ \text { Fabric: } & 7\end{array}$

Object ID: AT13/5/1/8 Tafel 6/4

Shape: base

Type: slightly inverted rim

Variant: flat

Type code: $\quad$ B2B

Preservation: abraded

Fabric: $\quad 4$

Dimensions (cm):

wall thickness: 1.1

diam. base: 12

diam. base, preserv. (\%): 10
Object ID: AT13/5/1/12

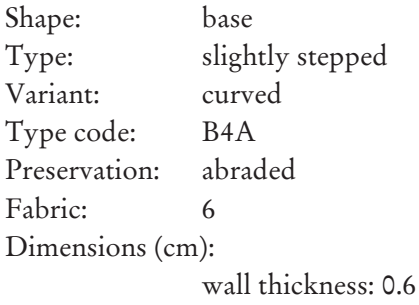

Vertical handle

Object ID: AT13/5/1/15

Shape: vertical handle

Type: oval cross section

Variant: undecorated

Type code: VH1B

Preservation: abraded

Fabric: 14

Dimensions (cm):

width: 2

thickness: 1.5

Decorated wall fragment

Object ID: AT13/5/1/16

Shape: decorated wall fragment

Type: Furchenstich

Type code: DF9

Preservation: abraded

Fabric: $\quad 10$

Description: bundle of three lines

Dimensions $(\mathrm{cm})$ :

wall thickness: 0.5

\section{Plastic cordon}

Object ID: AT13/5/1/13

Shape: plastic cordon

Type: simple

Variant: open circle

Type code: $\quad$ PC2B

Preservation: abraded

Fabric: 2

Application: PC2B

Description: plastic cordon with unclear position

Dimensions (cm):

wall thickness: 1.2

diam. base: 9.7

Object ID: AT13/5/1/14 Tafel 6/5

Shape: plastic cordon

Type: $\quad$ finger impressions - unclear position

Variant: vertical lug

Type code: $\quad$ PC3A

Preservation: abraded

Fabric: $\quad 1$

Description: lastic cordon with finger impressions and knob

Dimensions $(\mathrm{cm})$ :

wall thickness: 1 


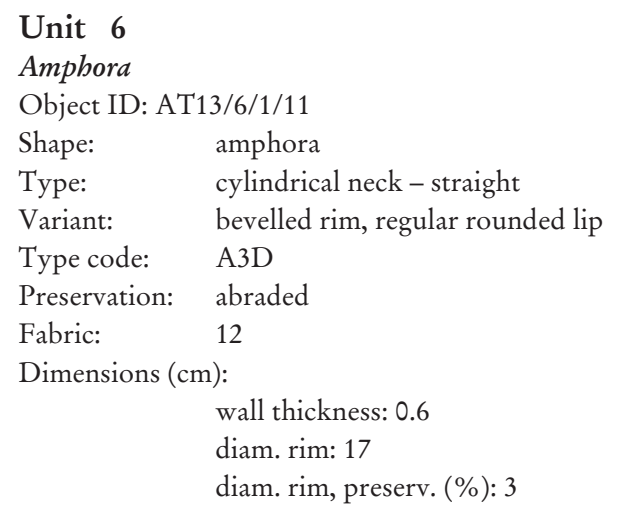

Object ID: AT13/6/1/4

Shape: amphora

Type: $\quad$ cylindrical neck - conical

Variant: $\quad$ regular rounded lip

Type code: A1A

Preservation: abraded

Fabric: $\quad 7$

Dimensions (cm):

$$
\begin{aligned}
& \text { wall thickness: } 0.6 \\
& \text { diam. rim: } 16 \\
& \text { diam. rim, preserv. (\%): } 8
\end{aligned}
$$

Object ID: AT13/6/1/5

Shape: amphora

Type: $\quad$ conical neck - bent outwards

Variant: $\quad$ regular rounded lip

Type code: A2A

Preservation: abraded

Fabric: $\quad 13$

Description: medium burnished surface

Dimensions (cm):

wall thickness: 0.8

diam. rim: 18

diam. rim, preserv. (\%): 14

\section{Jar}

$\begin{array}{ll}\text { Object ID: AT13/6/1/1 } & \text { Tafel 7/1 } \\ \text { Shape: } & \text { jar } \\ \text { Type: } & \text { narrow mouthed - concave } \\ \text { Variant: } & \text { squared lip } \\ \text { Type code: } & \text { J2E } \\ \text { Preservation: } & \text { abraded } \\ \text { Fabric: } & 4 \\ \text { Application: } & \text { PC1A } \\ \text { Description: } & \text { plastic cordon with finger impressions } \\ \text { Dimensions (cm): } & \\ & \text { wall thickness: } 1.1 \\ & \text { diam. rim: } 40 \\ & \text { diam. rim, preserv. }(\%): 9\end{array}$

Object ID: AT13/6/1/7

Shape: jar

Type: narrow mouthed - conical

Variant: bevelled rim, regular rounded lip

Type code: J2A
Preservation: abraded
Fabric: 12
Dimensions (cm):

wall thickness: 0.8
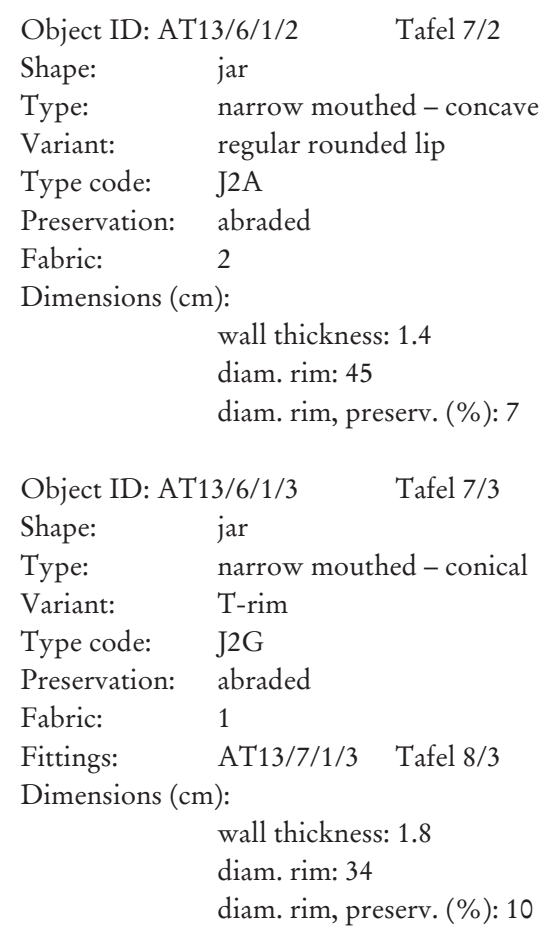

Shallow bowl

Object ID: AT13/6/1/6 Tafel 7/4

Shape: shallow bowl

Type: slightly inverted rim

Variant: thinned lip

Type code: $\quad$ SB2C

Fabric: $\quad 4$

Application: DF9, DF14

Description: Spiral motive; on the left: cut-out triangle Dimensions (cm): wall thickness: 0.9

\section{Baking pan}

Object ID: AT13/6/1/27 Tafel 8/1

Shape: baking pan

Type code: BP

Preservation: secondary burn

Fabric:

Dimensions $(\mathrm{cm})$ :

$$
\text { wall thickness: } 2.9
$$

Open vessel

Object ID: AT13/6/1/8

Shape: open vessel

Variant: $\quad$ regular rounded lip

Type code: Ov1A

Preservation: abraded

Fabric: $\quad 10$

Dimensions (cm): 


\section{Closed vessel}

Object ID: AT13/6/1/9

Shape: closed vessel

Variant: $\quad$ regular rounded lip

Type code: $\quad$ CvA

Preservation: abraded

Fabric: $\quad 6$

Dimensions $(\mathrm{cm})$ :

wall thickness: 0.9

Object ID: AT13/6/1/10

Shape: closed vessel

Variant: $\quad$ regular rounded lip

Type code: CvA

Preservation: abraded

Fabric: $\quad 4$

Description: medium burnished surface- outside Dimensions (cm):

wall thickness: 0.6

Object ID: AT13/6/1/22

Shape: closed vessel

Type: not definable

Type code: CVOO

Preservation: secondary burn

Fabric: $\quad 10$

Application: DF2, VH1B

Description: bundle of lines at right angle

Dimensions (cm):

width: 2.4

thickness: 1.9

wall thickness: 0.7

\section{Base}

Object ID: AT13/6/1/12

Shape: base

Type: flat base

Variant: regular transition

Type code: B2B

Preservation: abraded

Fabric: 1

Dimensions $(\mathrm{cm})$ :

wall thickness: 1.1

diam. base: 10

diam. base, preserv. (\%): 25

Object ID: AT13/6/1/13

Shape: base

Type: slightly stepped

Variant: curved

Type code: B4A

Preservation: abraded

Fabric: $\quad 5$

Dimensions (cm):

wall thickness: 0.5

diam. base: 10

diam. base, preserv. (\%): 23

Vertical handle

Object ID: AT13/6/1/18

Shape: vertical handle

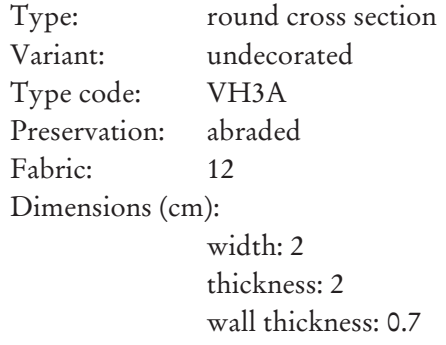

Object ID: AT13/6/1/21

$\begin{array}{ll}\text { Shape: } & \text { vertical handle } \\ \text { Type: } & \text { oval cross section } \\ \text { Variant: } & \text { undecorated } \\ \text { Type code: } & \text { VH1B } \\ \text { Preservation: } & \text { abraded } \\ \text { Fabric: } & 2 \\ \text { Dimensions (cm): } & \\ & \text { width: } 2.5 \\ & \text { thickness: } 2\end{array}$

Object ID: AT13/6/1/26

Shape: vertical handle

Type: not definable

Type code: VHO

Preservation: abraded

Description: coarse surface, roughly smoothed, medium tempered, orange-red

Dimensions (cm):

width: 5

thickness: 3

Object ID: AT13/6/1/19

Shape: vertical handle

Type: oval cross section

Variant: undecorated

Type code: VH1B

Preservation: abraded

Fabric: $\quad 12$

Dimensions $(\mathrm{cm})$ :

width: 2.7

thickness: 2.5

Object ID: AT13/6/1/14

$\begin{array}{ll}\text { Shape: } & \text { vertical handle } \\ \text { Type: } & \text { oval cross section } \\ \text { Variant: } & \text { undecorated } \\ \text { Type code: } & \text { VH1B } \\ \text { Preservation: } & \text { abraded } \\ \text { Fabric: } & 3 \\ \text { Dimensions (cm): } & \\ & \text { width: } 2.5 \\ & \text { thickness: } 1 \\ & \text { wall thickness: } 0.6\end{array}$

Object ID: AT13/6/1/17

Shape: vertical handle

Type: oval cross section

Variant: undecorated

Type code: VH1B

Preservation: abraded 

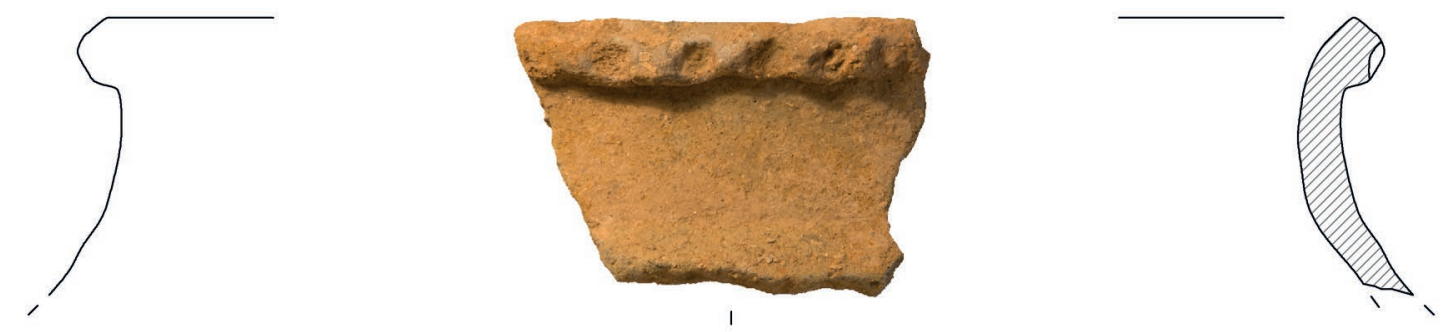

1
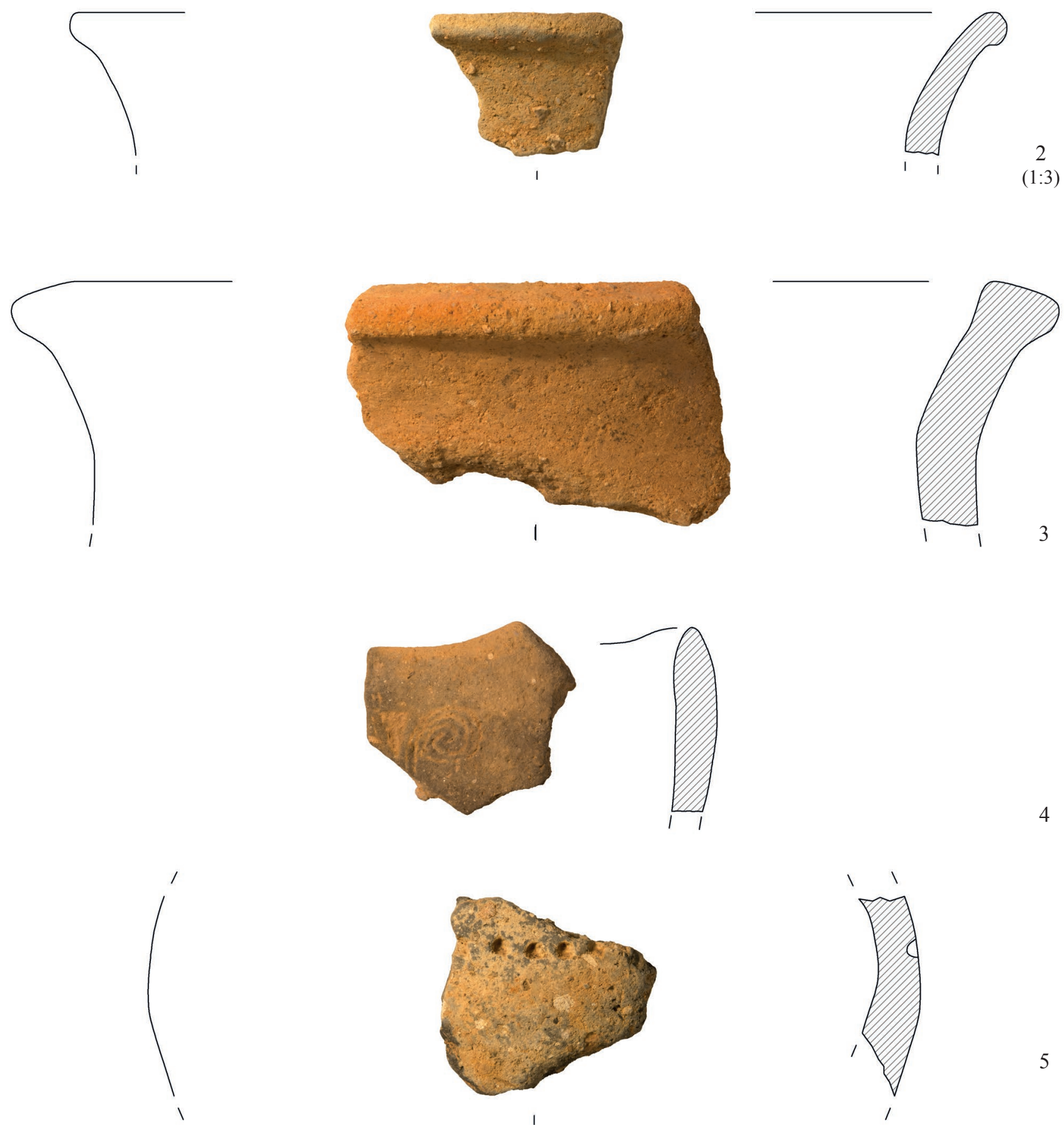

Tafel 7. Ada Tepe, Nordostquartier, Haus 7. Keramik. - 1-2. SE 06 (1:3). - 3-5. SE 06 (1:2) (Zeichnungen: S. Eder, B. Horejs, St. Horvath, D. Kolic, S. Molla-Djafari, M. Röcklinger; Fotos: Th. Urban). 
Fabric: 3

Dimensions (cm):

width: 3.6

thickness: 2.5

Object ID: AT13/6/1/16

Shape: vertical handle

Type: rhombic cross section

Variant: undecorated

Type code: VH2A

Preservation: abraded

Fabric: 3

Dimensions $(\mathrm{cm})$ :

width: 3.5

thickness: 2.5

wall thickness: 0.8

Object ID: AT13/6/1/15

Shape: vertical handle

Type: oval cross section

Variant: undecorated

Type code: VH1B

Preservation: abraded

Fabric:

10

Dimensions (cm):

width: 2.4

thickness: 1.5

Object ID: AT13/6/1/20

Shape: vertical handle

Type: oval cross section

Variant: undecorated

Type code: VH1B

Preservation: abraded

Fabric: 6

Dimensions $(\mathrm{cm})$ :

width: 3

thickness: 2

wall thickness: 0.6

Decorated wall fragment

Object ID: AT13/6/1/24

Shape: decorated wall fragment

Type: incrusted

Variant: grooved

Type code: DF1E

Preservation: abraded

Fabric: 5

Dimensions $(\mathrm{cm})$ : wall thickness: 0.7

Object ID: AT13/6/1/25 Tafel 7/5

Shape: decorated wall fragment

Type: impressed

Variant: dots

Type code: DF4B

Preservation: abraded

Fabric: 4

Description: Impressed dots

Dimensions (cm):

wall thickness: 1.2
Unit 7

Amphora

Object ID: AT13/7/1/1 Tafel 8/2

Shape: amphora

Type: conical neck - bent outwards

Variant: regular rounded lip

Type code: A2A

Preservation: secondary burn

Fabric: 4

Dimensions (cm):

wall thickness: 1

diam. rim: 23

diam. rim, preserv. (\%): 5.5

Object ID: AT13/7/1/2

Shape: amphora

Type: $\quad$ cylindrical neck - conical

Variant: bevelled rim, regular rounded lip

Type code: A1D

Preservation: abraded

Fabric: 6

Dimensions (cm):

wall thickness: 0.9

diam. rim: 14

diam. rim, preserv. (\%): 8

Object ID: AT13/7/1/8

Shape: amphora

Type: $\quad$ cylindrical neck - conical

Variant: slightly thickened lip

Type code: A1B

Preservation: secondary burn

Fabric: $\quad 5$

Description: outer surface medium burnished

Dimensions $(\mathrm{cm})$ :

wall thickness: 1.1

Object ID: AT13/7/1/9

Shape: amphora

Type: $\quad$ cylindrical neck - straight

Variant: bead rim

Type code: $\quad \mathrm{A} 3 \mathrm{H}$

Preservation: abraded

Fabric: $\quad 3$

Dimensions (cm):

wall thickness: 0.8

diam. rim: 20

diam. rim, preserv. (\%): 10

Object ID: AT13/7/1/16

Shape: amphora

Type: $\quad$ cylindrical neck - conical

Variant: $\quad$ regular rounded lip

Type code: A1A

Preservation: abraded

Fabric: 6

Description: attached handle on the rim?

Dimensions (cm):

wall thickness: 0.8

diam. rim: 12

diam. rim, preserv. (\%): 26 

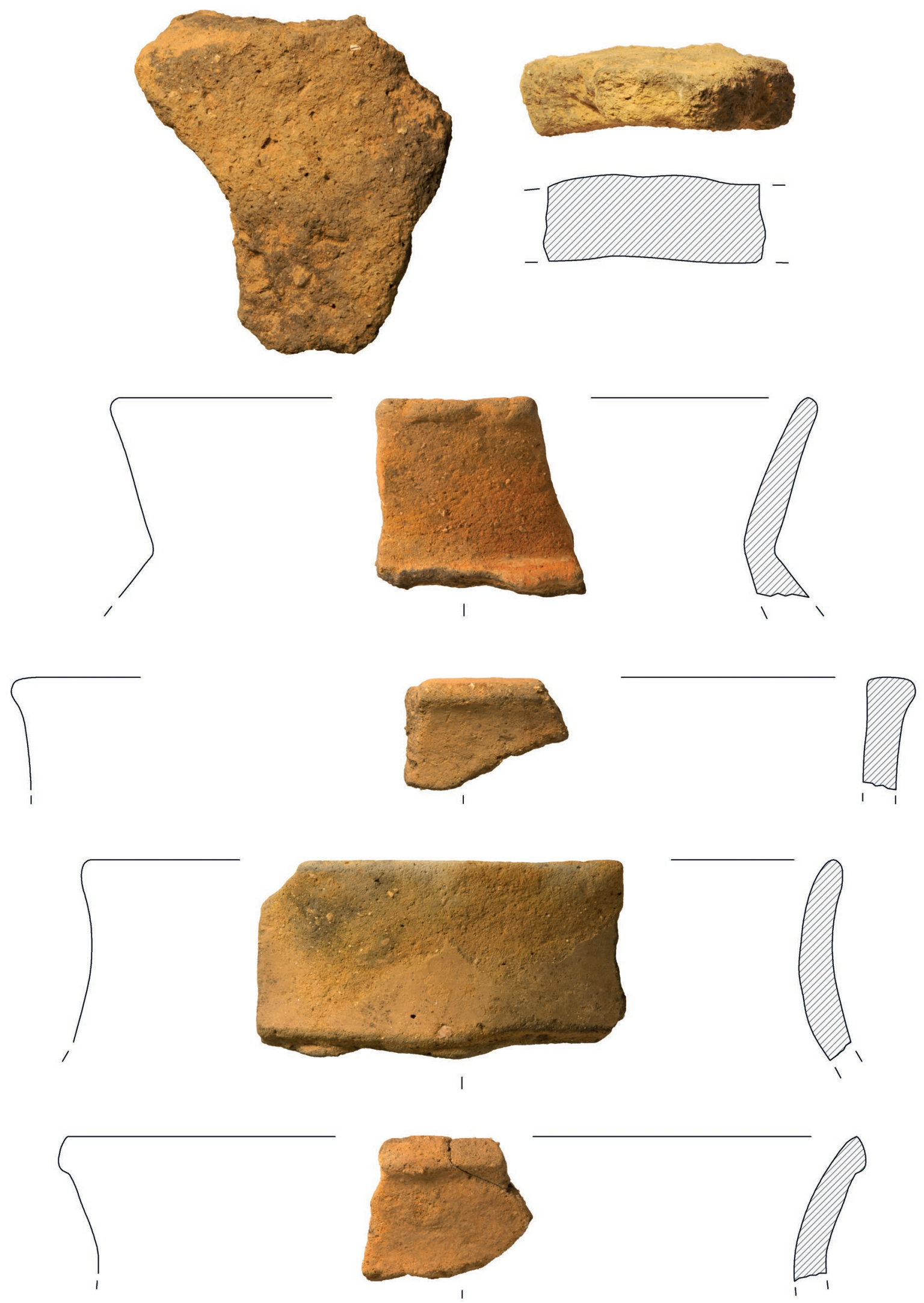

Tafel 8. Ada Tepe, Nordostquartier, Haus 7. Keramik. - 1. SE 06 (1:2). - 2, 4. SE 07 (1:2). - 3, 5. SE 07 (1:3) (Zeichnungen: S. Eder, B. Horejs, St. Horvath, D. Kolic, S. Molla-Djafari, M. Röcklinger; Fotos: Th. Urban). 
Jar

Object ID: AT13/7/1/7 Tafel 8/4

Shape: jar

Type: narrow mouthed - conical

Variant: $\quad$ regular rounded lip

Type code: J2A

Preservation: secondary burn

Fabric: 7

Description: outer surface and inner surface (upper part)

Dimensions (cm): medium burnished

wall thickness: 1.4

diam. rim: 24

diam. rim, preserv. (\%): 12

Object ID: AT13/7/1/12

Shape: jar

Type: narrow mouthed - concave

Variant: regular rounded lip

Type code: J1A

Preservation: abraded

Fabric: 1

Application: PC1A

Dimensions (cm):

wall thickness: 1.3

Object ID: AT13/7/1/10

Shape: jar

Type: narrow mouthed - conical

Variant: slightly thickened lip

Type code: J2B

Preservation: abraded

Fabric: $\quad 4$

Description: surface roughly smoothened

Dimensions (cm):

wall thickness: 1.4

diam. rim: 28

diam. rim, preserv. (\%): 5

Object ID: AT13/7/1/13

$\begin{array}{ll}\text { Shape: } & \text { jar } \\ \text { Type: } & \text { narrow mouthed - concave } \\ \text { Variant: } & \text { regular rounded lip } \\ \text { Type code: } & \text { J1A } \\ \text { Preservation: } & \text { abraded } \\ \text { Fabric: } & 2 \\ \text { Application: } & \text { PC1A } \\ \text { Dimensions (cm): } & \\ & \text { wall thickness: } 1.3 \\ & \text { diam. rim: } 20 \\ & \text { diam. rim, preserv. }(\%): 6\end{array}$

Object ID: AT13/7/1/15 Tafel 1/5

$\begin{array}{ll}\text { Shape: } & \text { jar } \\ \text { Type: } & \text { narrow mouthed - concave } \\ \text { Variant: } & \text { bead rim } \\ \text { Type code: } & \text { J2H } \\ \text { Preservation: } & \text { abraded } \\ \text { Fabric: } & 3\end{array}$

Description: outer surface roughly smoothed Dimensions $(\mathrm{cm})$ :

wall thickness: 1.5

diam. rim: 40

diam. rim, preserv. (\%): 5

Object ID: AT13/7/1/21

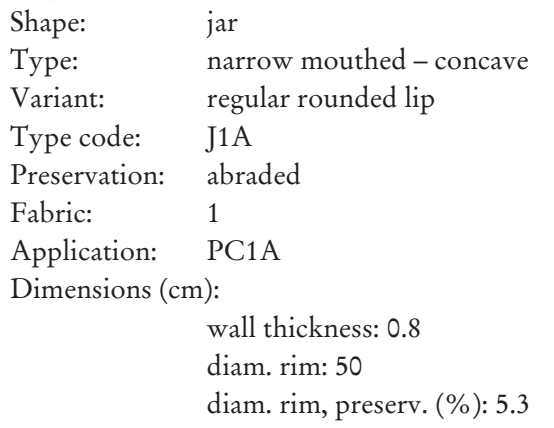

Beaker

Object ID: AT13/7/1/5 Tafel 9/1

Shape: beaker

Type: narrow mouthed

Variant: thinned lip

Type code: Be1C

Preservation: abraded

Fabric: $\quad 10$

Application: DF9

Description: two lines at the rim turning at right angle; small spiral

Dimensions ( $\mathrm{cm}$ ):

wall thickness: 0.4

Open vessel

Object ID: AT13/7/1/14

Shape: open vessel

Variant: $\quad$ regular rounded lip

Type code: Ov1A

Preservation: abraded

Fabric: $\quad 4$

Dimensions (cm):

wall thickness: 0.8

Closed vessel

Object ID: AT13/7/1/18

Shape: closed vessel

Variant: regular rounded lip

Type code: CvA

Preservation: abraded

Fabric: $\quad 4$

Dimensions (cm):

wall thickness: 0.9

Rim

Object ID: AT13/7/1/19

Shape: rim

Type: not definable

Variant: regular rounded lip

Type code: ROA 

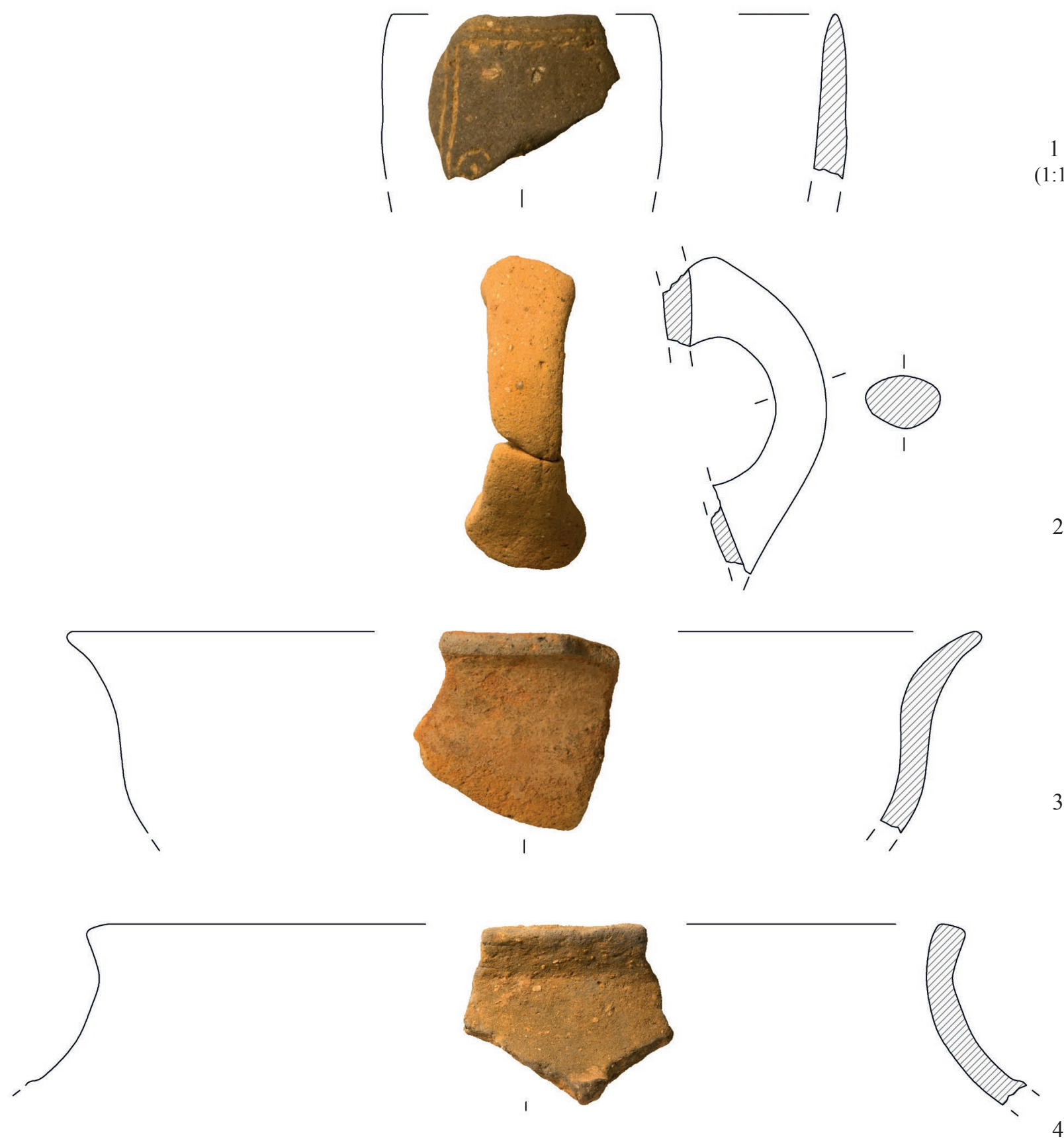

5
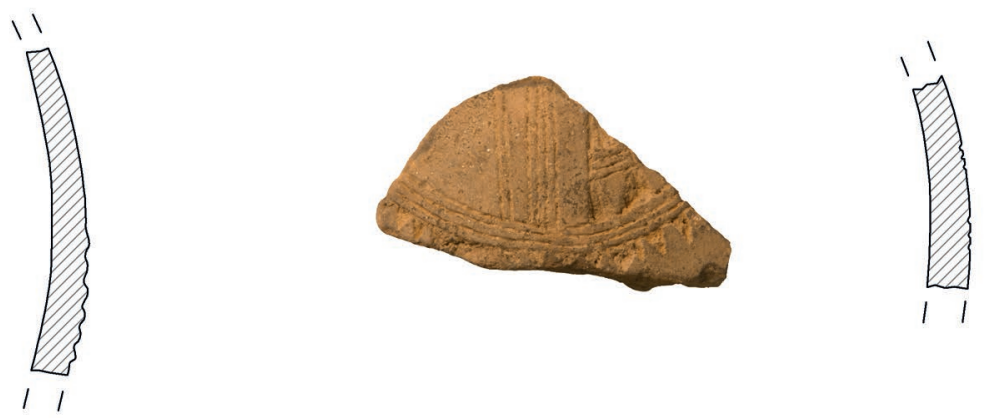

Tafel 9. Ada Tepe, Nordostquartier, Haus 7. Keramik. -1. SE 07 (1:1). -2. SE 07 (1:2). - 3-6. SE 08 (1:2) (Zeichnungen: S. Eder, B. Horejs, St. Horvath, D. Kolic, S. Molla-Djafari, M. Röcklinger; Fotos: Th. Urban). 
Preservation: abraded

Fabric:

Dimensions (cm):

wall thickness: 1.4

Object ID: AT13/7/1/20

Shape: $\quad$ rim

Type: not definable

Variant: regular rounded lip

Type code: ROA

Preservation: abraded

Fabric: 3

Dimensions ( $\mathrm{cm})$ :

wall thickness: 0.8

Object ID: AT13/7/1/4

Shape: rim

Type: not definable

Variant: regular rounded lip

Type code: ROA

Preservation: abraded

Fabric: 13

Dimensions (cm):

wall thickness: 0.6

\section{Base}

Object ID: AT13/7/1/24

Shape: base

Type: flat base

Variant: regular transition

Type code: B2B

Preservation: abraded

Fabric: 1

Dimensions (cm):

wall thickness: 1.2

Object ID: AT13/7/1/22

Shape: base

Type: slightly stepped

Variant: curved

Type code: B4A

Preservation: abraded

Fabric: $\quad 5$

Dimensions $(\mathrm{cm})$ :

wall thickness: 1

diam. base: 10

diam. base, preserv. (\%): 26

Object ID: AT13/7/1/23

Shape: base

Type: flat base

Variant: regular transition

Type code: B2B

Preservation: abraded

Fabric: 12

Dimensions $(\mathrm{cm})$ :

wall thickness: 0.8
Object ID: AT13/7/1/27

Shape: base

Type: stepped base

Variant: curved

Type code: B1A

Preservation: abraded

Fabric: 1

Dimensions (cm):

wall thickness: 1.3

diam. base: 16

diam. base, preserv. (\%): 14

Object ID: AT13/7/1/26

Shape: base

Type: flat base

Variant: regular transition

Type code: B2B

Preservation: abraded

Fabric: $\quad 14$

Dimensions (cm):

wall thickness: 0.8

diam. base: 7

diam. base, preserv. (\%): 100

Object ID: AT13/7/1/25

Shape: base

Type: stepped base

Variant: curved

Type code: B1A

Preservation: abraded

Fabric: $\quad 1$

Dimensions (cm):

wall thickness: 1.4

diam. base: 12

diam. base, preserv. (\%): 19

\section{Vertical handle}

Object ID: AT13/7/1/40

Shape: vertical handle

Type: round cross section

Variant: undecorated

Type code: VH3A

Preservation: abraded

Fabric: $\quad 1$

Dimensions (cm):

width: 3.1

Object ID: AT13/7/1/29

Shape: vertical handle

Type: oval cross section

Variant: undecorated

Type code: VH1B

Preservation: abraded

Fabric: $\quad 5$

Dimensions ( $\mathrm{cm})$ :

width: 4.1

thickness: 2.6

wall thickness: 0.8 
Object ID: AT13/7/1/28

$\begin{array}{ll}\text { Shape: } & \text { vertical handle } \\ \text { Type: } & \text { rhombic cross section } \\ \text { Variant: } & \text { undecorated } \\ \text { Type code: } & \text { VH2A } \\ \text { Preservation: } & \text { abraded } \\ \text { Fabric: } & 5 \\ \text { Dimensions (cm): } & \\ & \text { width: } 3.9 \\ & \text { thickness: } 2.5 \\ & \text { wall thickness: } 1.4\end{array}$

Object ID: AT13/7/1/30

Shape: vertical handle

Type: oval cross section

Variant: undecorated

Type code: VH1B

Preservation: abraded

Fabric: $\quad 2$

Dimensions (cm):

width: 3.6

thickness: 2.9

Object ID: AT13/7/1/31

Shape: vertical handle

Type: oval cross section

Variant: undecorated

Type code: VH1B

Preservation: abraded

Fabric: 14

Description: strap handle

Dimensions ( $\mathrm{cm})$ :

width: 3.2

thickness: 0.8

Object ID: AT13/7/1/32

Shape: vertical handle

Type: oval cross section

Variant: undecorated

Type code: VH1B

Preservation: abraded

Fabric: $\quad 3$

Dimensions (cm):

width: 3.2

thickness: 2.4

Object ID: AT13/7/1/33

Shape: vertical handle

Type: oval cross section

Variant: undecorated

Type code: VH1B

Preservation: abraded

Fabric: 6

Dimensions $(\mathrm{cm})$ :

width: 2.6

thickness: 1.8

Object ID: AT13/7/1/34

Shape: vertical handle

Type: oval cross section

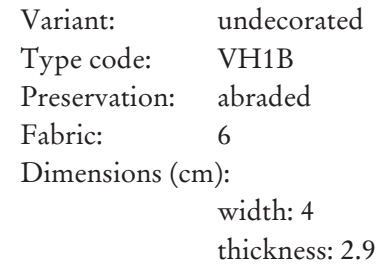

Fabric:

Dimensions $(\mathrm{cm})$ :

width: 4

thickness: 2.9

Object ID: AT13/7/1/43

Shape: vertical handle

Type: oval cross section

Variant: undecorated

Type code: VH1B

Preservation: abraded

Fabric: 2

Dimensions $(\mathrm{cm})$ :

\author{
width: 3.6 \\ thickness: 2.7
}

Object ID: AT13/7/1/36

Shape: vertical handle

Type: oval cross section

Variant: undecorated

Type code: VH1B

Preservation: abraded

Fabric:

6

Dimensions (cm):

$$
\begin{aligned}
& \text { width: } 1.8 \\
& \text { thickness: } 1.4
\end{aligned}
$$

Object ID: AT13/7/1/44

Shape: vertical handle

Type: oval cross section

Variant: decorated

Type code: VH1A

Preservation: abraded

Fabric: $\quad 6$

Application: DF7

Description: lines vertically and horizontally,

Dimensions (cm): forming a rectangle

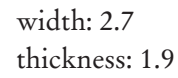

Object ID: AT13/7/1/41 Tafel 9/2

Shape: vertical handle

Type: flattened - oval

Variant: undecorated

Type code: VH4A

Preservation: abraded

Fabric: $\quad 14$

Description: construction plug

Dimensions (cm):

$$
\begin{aligned}
& \text { length: } 9.4 \\
& \text { width: } 2.1 \\
& \text { thickness: } 1.6
\end{aligned}
$$

Object ID: AT13/7/1/39

Shape: vertical handle

Type: oval cross section 


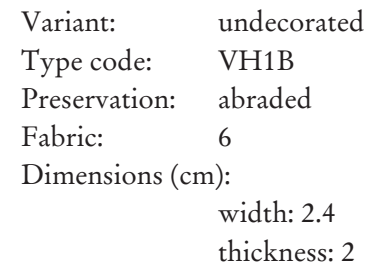

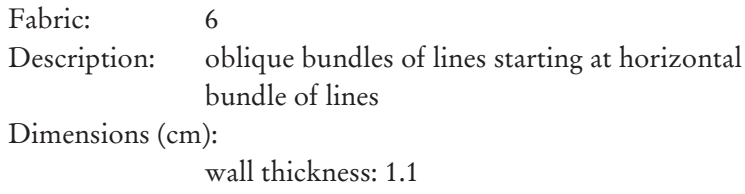

Object ID: AT13/7/1/46

Shape: decorated wall fragment

Type: $\quad$ grooved and Furchenstich

Type code: DF6

Preservation: abraded

Fabric: $\quad 10$

Description: bundle of lines with partly shaded lines Dimensions $(\mathrm{cm})$ : wall thickness: 0.6

Object ID: AT13/7/1/48

Shape: decorated wall fragment

Type: Furchenstich

Type code: DF9

Preservation: abraded

Fabric: 13

Description: triangular motive of lines

Dimensions $(\mathrm{cm})$ :

wall thickness: 0.8

\section{Unit 8}

Amphora

Object ID: AT12/8/1/3

Shape: amphora

Type: not definable

Variant: $\quad$ regular rounded lip

Type code: AOA

Preservation: abraded

Fabric: 6

Dimensions (cm):

$$
\text { wall thickness: } 1.1
$$

Object ID: AT12/8/1/4

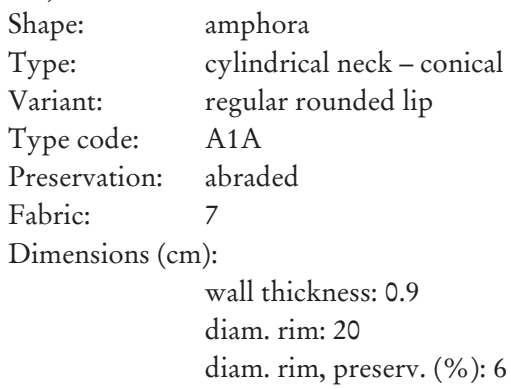




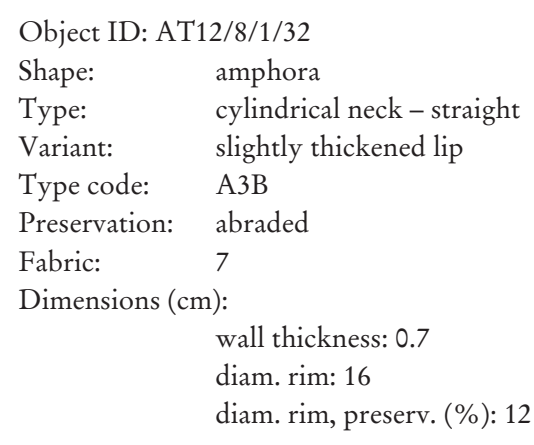

Object ID: AT12/8/1/11

Shape: amphora

Type: $\quad$ conical neck - bent outwards

Variant: regular rounded lip

Type code: A2A

Preservation: abraded

Fabric: $\quad 5$

Object ID: AT12/8/1/12

Shape: amphora

Type: not definable

Variant: slightly thickened lip

Type code: AOB

Preservation: abraded

Fabric: $\quad 6$

Dimensions (cm):

wall thickness: 0.8

Jar

Object ID: AT12/8/1/10

Shape: jar

Type: narrow mouthed - conical

Variant: regular rounded lip

Type code: J2A

Preservation: abraded

Fabric: 6

Dimensions (cm):

wall thickness: 0.9

diam. rim: 30

diam. rim, preserv. (\%): 6

Object ID: AT12/8/1/1

Shape: jar

Type: narrow mouthed - concave

Variant: regular rounded lip

Type code: J1A

Preservation: abraded

Fabric: $\quad 4$

Application: PC1A

Description: angular finger impressions

Dimensions ( $\mathrm{cm})$ :

wall thickness: 0.7

diam. rim: 26

diam. rim, preserv. (\%): 4

Object ID: AT12/8/1/2

Shape: jar

Type: narrow mouthed - concave

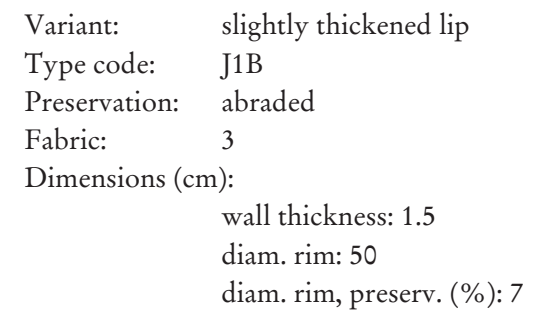

Object ID: AT12/8/1/28 Tafel 9/4

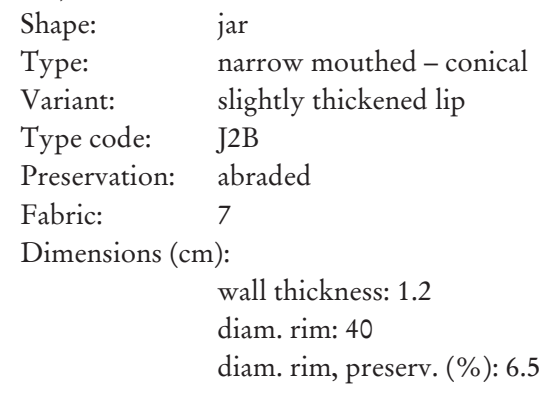

Shallow bowl

Object ID: AT12/8/1/8 Tafel 9/3

Shape: shallow bowl

Type: s-shaped

Variant: thinned lip

Type code: SB1C

Preservation: abraded

Fabric: $\quad 12$

Dimensions (cm):

wall thickness: 0.7

diam. rim: 27

diam. rim, preserv. (\%): 3.5

Closed vessel

Object ID: AT12/8/1/5

Shape: closed vessel

Variant: regular rounded lip

Type code: CvA

Preservation: abraded

Fabric: 1

Dimensions (cm): wall thickness: 1.2

Object ID: AT12/8/1/7

Shape: closed vessel

Variant: $\quad$ regular rounded lip

Type code: CvA

Preservation: abraded

Fabric: $\quad 5$

Dimensions $(\mathrm{cm})$ : wall thickness: 1.1

Object ID: AT12/8/1/29 Tafel 9/5

Shape: closed vessel

Type code: CVo

Preservation: abraded

Fabric: $\quad 10$

Application: DF7

Description: Possibly triangular bundle of lines. The lines within are connected to each other by short lines 
Dimensions (cm): wall thickness: 0.6

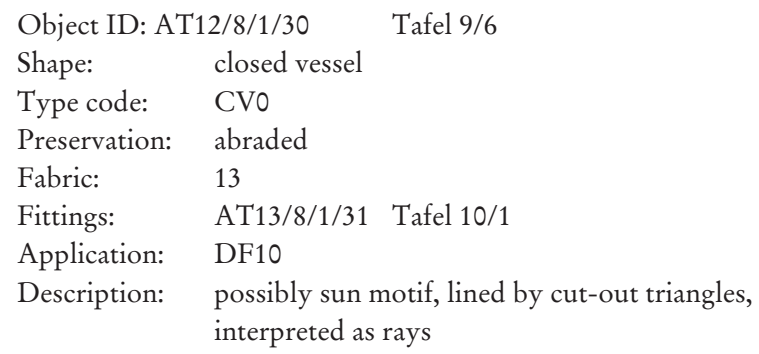

Dimensions (cm):

wall thickness: 0.9

\section{Base}

Object ID: AT12/8/1/16

Shape: base

Type: flat base

Variant: regular transition

Type code: B2B

Preservation: abraded

Fabric:

Dimensions $(\mathrm{cm})$ :

wall thickness: 1.2

\section{Vertical handle}

Object ID: AT12/8/1/24

Shape: vertical handle

Type: oval cross section

Variant: undecorated

Type code: VH1B

Preservation: abraded

Fabric: 7

Dimensions $(\mathrm{cm})$ :

width: 3.5

thickness: 2.2

wall thickness: 0.9

Object ID: AT12/8/1/23

Shape: vertical handle

Type: rhombic cross section

Variant: undecorated

Type code: $\quad$ VH2A

Preservation: abraded

Fabric:

Dimensions ( $\mathrm{cm})$ :

width: 2.4

thickness: 1.9

Object ID: AT12/8/1/20

Shape: vertical handle

Type: oval cross section

Variant: undecorated

Type code: VH1B

Preservation: abraded

Fabric: 1

Dimensions $(\mathrm{cm})$ :

width: 3.5

thickness: 2.3

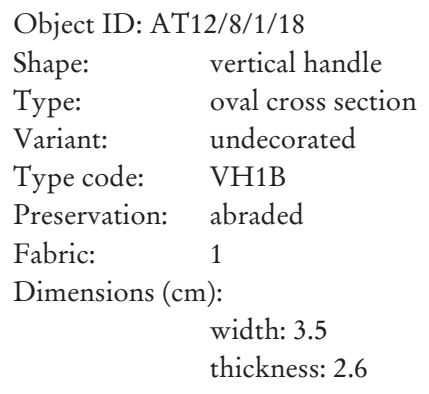

\section{Handle-undefined}

Object ID: AT12/8/1/22

Shape: handle-undefined

Type: oval cross section

Variant: undecorated

Type code: H1A

Preservation: abraded

Fabric: $\quad 12$

Dimensions $(\mathrm{cm})$ :

width: 1.8

thickness: 1.4

Object ID: AT12/8/1/21

Shape: handle-undefined

Type: oval cross section

Variant: undecorated

Type code: H1A

Preservation: abraded

Fabric: $\quad 1$

Dimensions $(\mathrm{cm})$ :

width: 3.4

thickness: 2

Object ID: AT12/8/1/19

Shape: handle - undefined

Type: oval cross section

Variant: undecorated

Type code: H1A

Preservation: abraded

Fabric: 2

Dimensions (cm):

width: 3.2

thickness: 2.6

Object ID: AT12/8/1/17

Shape: handle-undefined

Type: oval cross section

Variant: undecorated

Type code: H1A

Preservation: abraded

Fabric: 1

Dimensions $(\mathrm{cm})$ :

width: 3.9

thickness: 3.3

Decorated wall fragment

Object ID: AT12/8/1/27

Shape: decorated wall fragment

Type: incised and Furchenstich 
Type code: DF7

Preservation: abraded

Fabric: $\quad 12$

Description: bundle of lines with dots running underneath

Dimensions (cm):

wall thickness: 0.7

Object ID: AT12/8/1/33

Shape: decorated wall fragment

Type: $\quad$ grooved and Furchenstich

Type code: DF6

Preservation: abraded

Fabric: 7

Description: Bundle of lines

Dimensions $(\mathrm{cm})$ :

wall thickness: 0.7

Object ID: AT12/8/1/25 Tafel 10/2

Shape: decorated wall fragment

Type: $\quad$ cut out and Furchenstich

Type code: DF10

Preservation: abraded

Fabric: $\quad 4$

Description: Triangular motive of lines with triangular cut-outs inside

Dimensions $(\mathrm{cm})$ :

wall thickness: 0.9

Object ID: AT12/8/1/26

Shape: decorated wall fragment

Type: grooved

Type code: DF3

Preservation: abraded

Fabric: $\quad 5$

Description: Bundle of lines

Dimensions $(\mathrm{cm})$ :

wall thickness: 0.4

\section{Unit 9}

\section{Amphora}

Object ID: AT13/9/1/5

Shape: amphora

Type: $\quad$ cylindrical neck - conical

Variant: slightly thickened lip

Type code: A1B

Preservation: secondary burn

Fabric: 6

Dimensions (cm):

wall thickness: 1.1

diam. rim: 24

diam. rim, preserv. (\%): 6

Object ID: AT13/9/1/15

Shape: amphora

Type: $\quad$ conical neck - bent outwards

Variant: regular rounded lip

Type code: A2A

Preservation: abraded

Fabric: 12
Dimensions $(\mathrm{cm})$ : wall thickness: 0.7

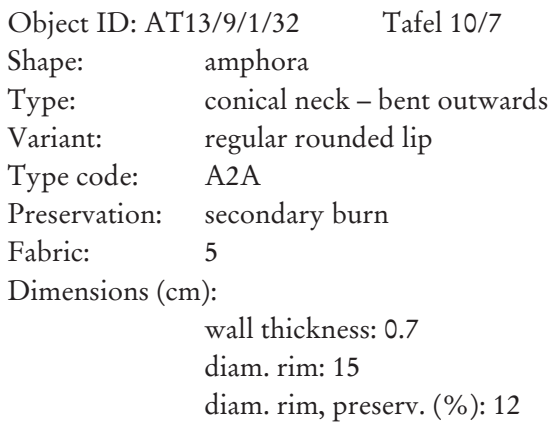

Object ID: AT13/9/1/1

Shape: amphora

Type: $\quad$ cylindrical neck - conical

Variant: bevelled rim, regular rounded lip

Type code: A1F

Preservation: abraded

Fabric: 6

Dimensions (cm): wall thickness: 1.1

\section{Jar}

Object ID: AT13/9/1/3 Tafel 10/3

Shape: jar

Type: narrow mouthed

Variant: regular rounded lip

Type code: J4A

Preservation: abraded

Fabric: 1

Application: PC1A

Description: angular finger impressions

Dimensions $(\mathrm{cm})$ :

wall thickness: 1.3

diam. rim: 34

diam. rim, preserv. (\%): 3.8

Object ID: AT13/9/1/31 Tafel 10/6

Shape: jar

Type: $\quad$ cylindrical neck - egg shaped

Variant: bead rim

Type code: J3H

Preservation: abraded

Fabric: $\quad 7$

Dimensions $(\mathrm{cm})$ :

wall thickness: 0.9

diam. rim: 22

diam. rim, preserv. (\%): 5

Deep bowl

Object ID: AT13/9/1/9

Shape: deep bowl

Type: funnel shaped neck

Variant: regular rounded lip

Type code: DB1A

Preservation: abraded 
Fabric: $\quad 12$

Dimensions (cm):

wall thickness: 0.6

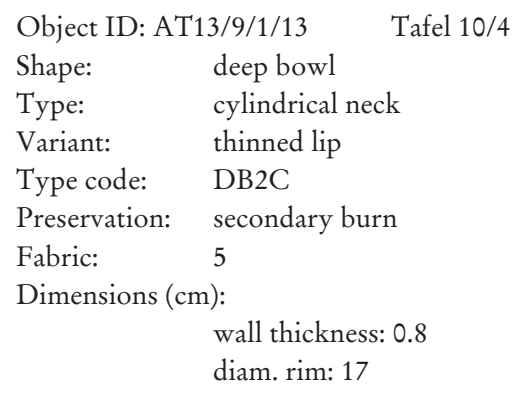

\section{Cup}

Object ID: AT13/9/1/33 Tafel 11/1

Shape: cup

Type: sloping rim

Type code: $\quad \mathrm{Cu} 1$

Preservation: abraded

Fabric: $\quad 5$

Application: DF6, VH1A

Description: handle attached to the rim and dragged upwards; handle: triangular motive of bundles of lines; body: bundles of lines, partially lines with oblique lines starting at the bundles

Dimensions $(\mathrm{cm})$ :

diam. body: 28

width: 5

thickness: 3.3

wall thickness: 0.7

\section{Open vessel}

Object ID: AT13/9/1/11

Shape: open vessel

Variant: $\quad$ regular rounded lip

Type code: OV1A

Preservation: abraded

Fabric: $\quad 10$

Dimensions (cm):

$$
\text { wall thickness: } 0.5
$$

\section{Closed vessel}

Object ID: AT13/9/1/16

Shape: closed vessel

Variant: regular rounded lip

Type code: CvA

Preservation: abraded

Fabric: $\quad 5$

Dimensions (cm): wall thickness: 0.9

Object ID: AT13/9/1/4

Shape: closed vessel

Variant: regular rounded lip

Type code: CvA

Preservation: abraded

Fabric: 5

Dimensions ( $\mathrm{cm})$ : wall thickness: 1
Object ID: AT13/9/1/10

Shape: closed vessel

Variant: regular rounded lip

Type code: CvA

Preservation: abraded

Fabric: $\quad 7$

Dimensions (cm): wall thickness: 0.9

Object ID: AT13/9/1/6

$\begin{array}{ll}\text { Shape: } & \text { closed vessel } \\ \text { Variant: } & \text { squared lip } \\ \text { Type code: } & \text { CvE } \\ \text { Preservation: } & \text { abraded } \\ \text { Fabric: } & 2 \\ \text { Dimensions (cm): }\end{array}$

Dimensions (cm):

wall thickness: 1.4

Object ID: AT13/9/1/7

Shape: closed vessel

Variant: $\quad$ regular rounded lip

Type code: CvA

Preservation: abraded

Fabric: 13

Dimensions (cm):

wall thickness: 0.6

Object ID: AT13/9/1/12

Shape: closed vessel

Variant: regular rounded lip

Type code: CvA

Preservation: abraded

Fabric: 6

Dimensions (cm):

wall thickness: 0.8

Base

Object ID: AT13/9/1/21

Shape: base

Type: slightly stepped

Variant: curved

Type code: B4A

Preservation: abraded

Fabric: $\quad 7$

Dimensions $(\mathrm{cm})$ :

wall thickness: 1.6

Object ID: AT13/9/1/17

Shape: base

Type: slightly stepped

Variant: curved

Type code: B4A

Preservation: abraded

Fabric: $\quad 3$

Dimensions (cm):

wall thickness: 1.4

diam. base: 11

diam. base, preserv. (\%): 22 
1
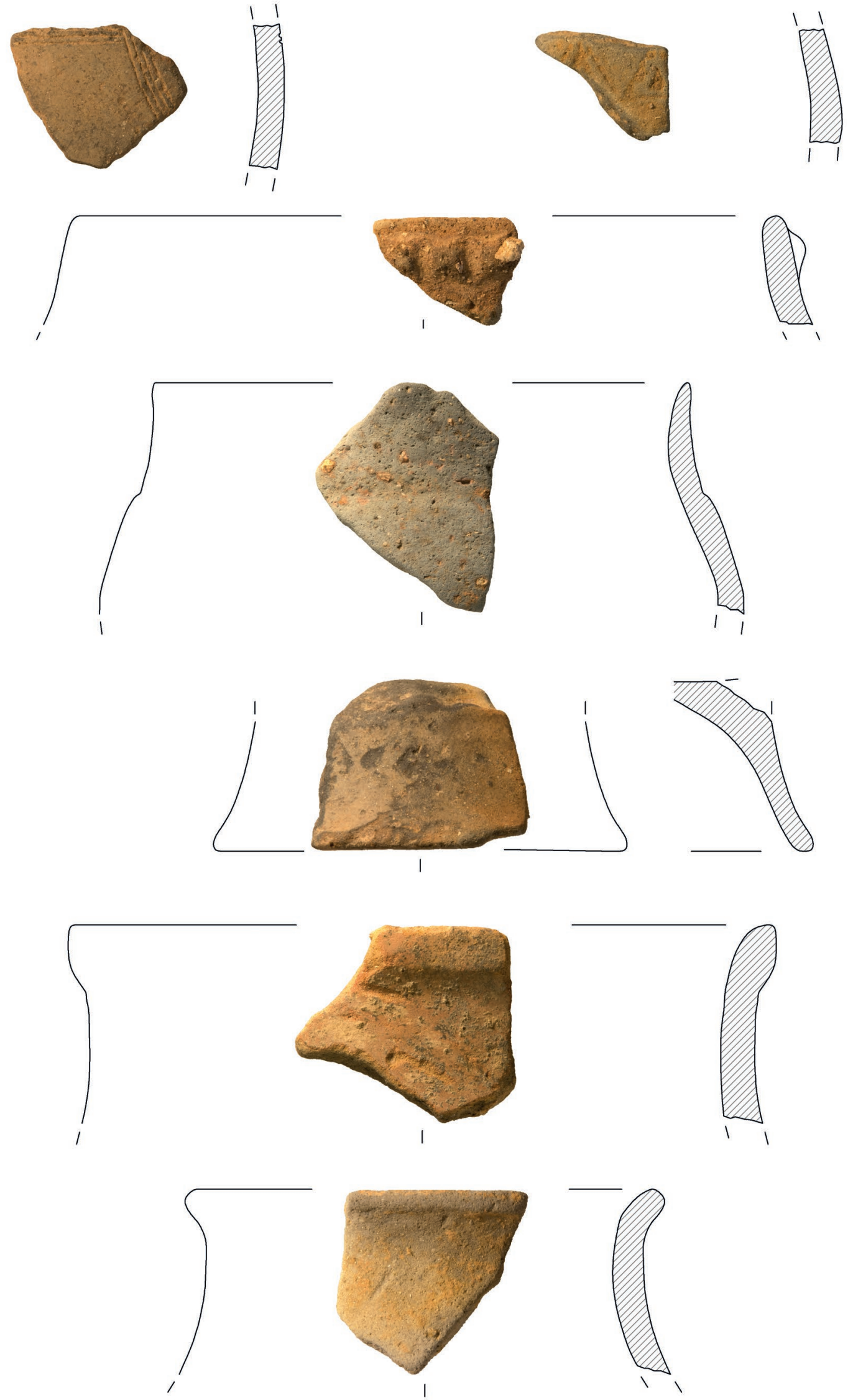

Tafel 10. Ada Tepe, Nordostquartier, Haus 7. Keramik. - 1-2. SE 08 (1:2). - 3-7. SE 09 (1:2) (Zeichnungen: S. Eder, B. Horejs, St. Horvath, D. Kolic, S. Molla-Djafari, M. Röcklinger; Fotos: Th. Urban). 
Object ID: AT13/9/1/18

Shape: base

Type: stepped base

Variant: curved

Type code: B1A

Preservation: abraded

Fabric: 1

Dimensions $(\mathrm{cm})$ :

wall thickness: 1.2

Object ID: AT13/9/1/19

Shape: base

Type: slightly stepped

Variant: curved

Type code: B4A

Preservation: abraded

Fabric: 1

Dimensions ( $\mathrm{cm})$ :

wall thickness: 1.8

diam. base: 12

diam. base, preserv. (\%): 26

Object ID: AT13/9/1/20

Shape: base

Type: flat base

Variant: regular transition

Type code: B2B

Preservation: abraded

Fabric:

Dimensions $(\mathrm{cm})$ :

wall thickness: 0.9

diam. base: 10

diam. base, preserv. (\%): 22

Object ID: AT13/9/1/22

Shape: base

Type: flat base

Variant: regular rounded lip

Type code: $\quad$ B2B

Preservation: abraded

Fabric: 6

Dimensions $(\mathrm{cm})$ :

wall thickness: 1.1

Object ID: AT13/9/1/30 Tafel 10/5

Shape: base

Type: ring base

Variant: concave

Type code: B5A

Preservation: secondary burn

Fabric: 13

Dimensions (cm):

wall thickness: 0.8

diam. base: 13

diam. base, preserv. (\%): 12

Object ID: AT13/9/1/23

Shape: base

Type: slightly stepped

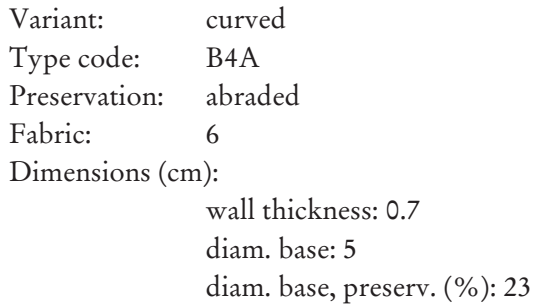

Vertical handle

Object ID: AT13/9/1/26

Shape: vertical handle

Type: oval cross section

Variant: undecorated

Type code: VH1B

Preservation: abraded

Fabric: $\quad 4$

Dimensions (cm):

width: 3.6

thickness: 2.1

Object ID: AT13/9/1/2

Shape: vertical handle

Type: oval cross section

Variant: undecorated

Type code: VH1B

Preservation: abraded

Fabric: $\quad 7$

Dimensions $(\mathrm{cm})$ :

width: 4

thickness: 2.1

Handle - undefined

Object ID: AT13/9/1/28

Shape: handle - undefined

Type: oval cross section

Variant: undecorated

Type code: H1A

Preservation: abraded

Fabric: $\quad 4$

Dimensions (cm):

width: 3.5

thickness: 2.7

Object ID: AT13/9/1/27

Shape: handle-undefined

Type: oval cross section

Variant: undecorated

Type code: H1A

Preservation: abraded

Fabric: $\quad 1$

Dimensions (cm):

width: 2.9

thickness: 2.5

Plastic cordon

Object ID: AT13/9/1/34

Shape: plastic cordon

Variant: unclear position - horizontal 


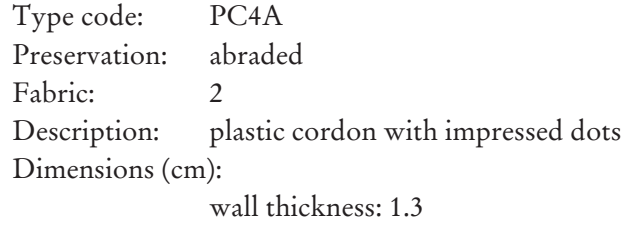

\section{Object ID: AT13/9/1/25}

Shape: plastic cordon

Type: finger impressed

Variant: unclear position - horizontal

Type code: PC1B

Preservation: abraded

Fabric: $\quad 2$

Dimensions (cm):

wall thickness: 1

\section{Unit 10}

Amphora

Object ID: AT12/10/1/7

Shape: amphora

Type: cylindrical neck - conical

Variant: regular rounded lip

Type code: A1A

Preservation: abraded

Fabric: $\quad 5$

Dimensions (cm):

wall thickness: 1.1

diam. rim: 12

diam. rim, preserv. (\%): 15

Object ID: AT12/10/1/1

\section{Shape: amphora}

Type: $\quad$ cylindrical neck - conical

Variant: bevelled rim, regular rounded lip

Type code: A1F

Preservation: abraded

Fabric: $\quad 13$

Dimensions (cm):

wall thickness: 0.9

diam. rim: 14

diam. rim, preserv. (\%): 5

\section{Object ID: AT12/10/1/13}

Shape: amphora

Type: $\quad$ cylindrical neck - straight

Variant: bevelled rim, regular rounded lip

Type code: A3F

Preservation: abraded

Fabric: $\quad 12$

Dimensions (cm):

wall thickness: 0.8

diam. rim: 20

diam. rim, preserv. (\%): 7

Object ID: AT12/10/1/4

Shape: amphora

Type: cylindrical neck - conical

Variant: bevelled rim, regular rounded lip

Type code: A1F
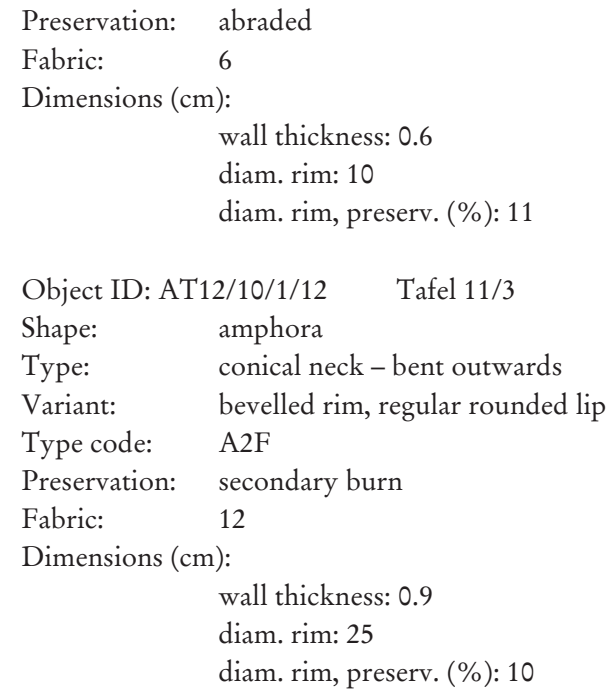

Jar

Object ID: AT12/10/1/33

Shape: jar

Type: not definable

Variant: regular rounded lip

Type code: JOA

Fabric: 1

Application: PC1A

Dimensions (cm):

wall thickness: 1

Object ID: AT12/10/1/8

Shape: jar

Type: narrow mouthed - concave

Variant: slightly thickened lip

Type code: J1B

Preservation: abraded

Fabric: $\quad 12$

Dimensions (cm):

wall thickness: 1.1

diam. rim: 30

diam. rim, preserv. (\%): 7

Object ID: AT12/10/1/6

Shape: jar

Type: narrow mouthed - concave

Variant: slightly thickened lip

Type code: J1B

Preservation: abraded

Fabric: 1

Dimensions $(\mathrm{cm})$ :

wall thickness: 1.3

diam. rim: 30

diam. rim, preserv. (\%): 6

Object ID: AT12/10/1/2

Shape: jar

Type: narrow mouthed

Variant: regular rounded lip

Type code: J4A 
Fabric: $\quad 1$

Application: PC1A

Description: Angular finger impressions.

Dimensions (cm):

wall thickness: 1.2

\section{Deep bowl}

Object ID: AT12/10/1/10

Shape: deep bowl

Type: funnel shaped neck

Variant: regular rounded lip

Type code: DB1A

Preservation: abraded

Fabric: 12

Dimensions (cm):

wall thickness: 0.8

diam. rim: 24

diam. rim, preserv. (\%): 5

Object ID: AT12/10/1/11

Shape: deep bowl

Type: cylindrical neck

Variant: regular rounded lip

Type code: DB2A

Preservation: abraded

Fabric: 12

Description: inner and outer surface medium burnished, secondarily burned inside

Dimensions $(\mathrm{cm})$ :

wall thickness: 0.9

diam. rim: 28

diam. rim, preserv. (\%): 8

Shallow bowl

Object ID: AT12/10/1/9 Tafel 11/2

Shape: shallow bowl

Type: slightly inverted rim

Variant: regular rounded lip

Type code: SB2A

Preservation: abraded

Fabric: $\quad 10$

Dimensions (cm):

wall thickness: 0.65

diam. rim: 23

diam. rim, preserv. (\%): 5

Closed vessel

Object ID: AT12/10/1/5

Shape: closed vessel

Variant: regular rounded lip

Type code: CvA

Preservation: abraded

Fabric: $\quad 10$

Dimensions (cm):

wall thickness: 0.7

Object ID: AT12/10/1/3

Shape: closed vessel

Variant: $\quad$ regular rounded lip

Type code: CvA $\begin{array}{ll}\text { Preservation: } & \text { abraded } \\ \text { Fabric: } & 1\end{array}$

\section{Rim}

Object ID: AT12/10/1/14

Shape: rim

Type: not definable

Variant: thinned lip

Type code: ROC

Preservation: abraded

Fabric: $\quad 12$

Dimensions (cm):

wall thickness: 0.6

Base

Object ID: AT12/10/1/18

Shape: base

Type: flat base

Variant: regular transition

Type code: B2B

Preservation: abraded

Fabric: $\quad 3$

Dimensions (cm):

wall thickness: 1.4

diam. base: 12

diam. base, preserv. (\%): 15

Object ID: AT12/10/1/15

Shape: base

Type: stepped base

Variant: curved

Type code: B1B

Fabric: $\quad 3$

Dimensions $(\mathrm{cm})$ :

wall thickness: 1.2

Object ID: AT12/10/1/17

Shape: base

Type: slightly stepped

Variant: curved

Type code: B4A

Preservation: abraded

Fabric: 2

Dimensions (cm):

wall thickness: 1.2

diam. base: 12

diam. base, preserv. (\%): 15

Object ID: AT12/10/1/19

Shape: base

Type: flat base

Variant: sharp transition

Type code: B2A

Preservation: abraded

Fabric: 6

Dimensions (cm):

wall thickness: 1

diam. base: 10

diam. base, preserv. (\%): 21 

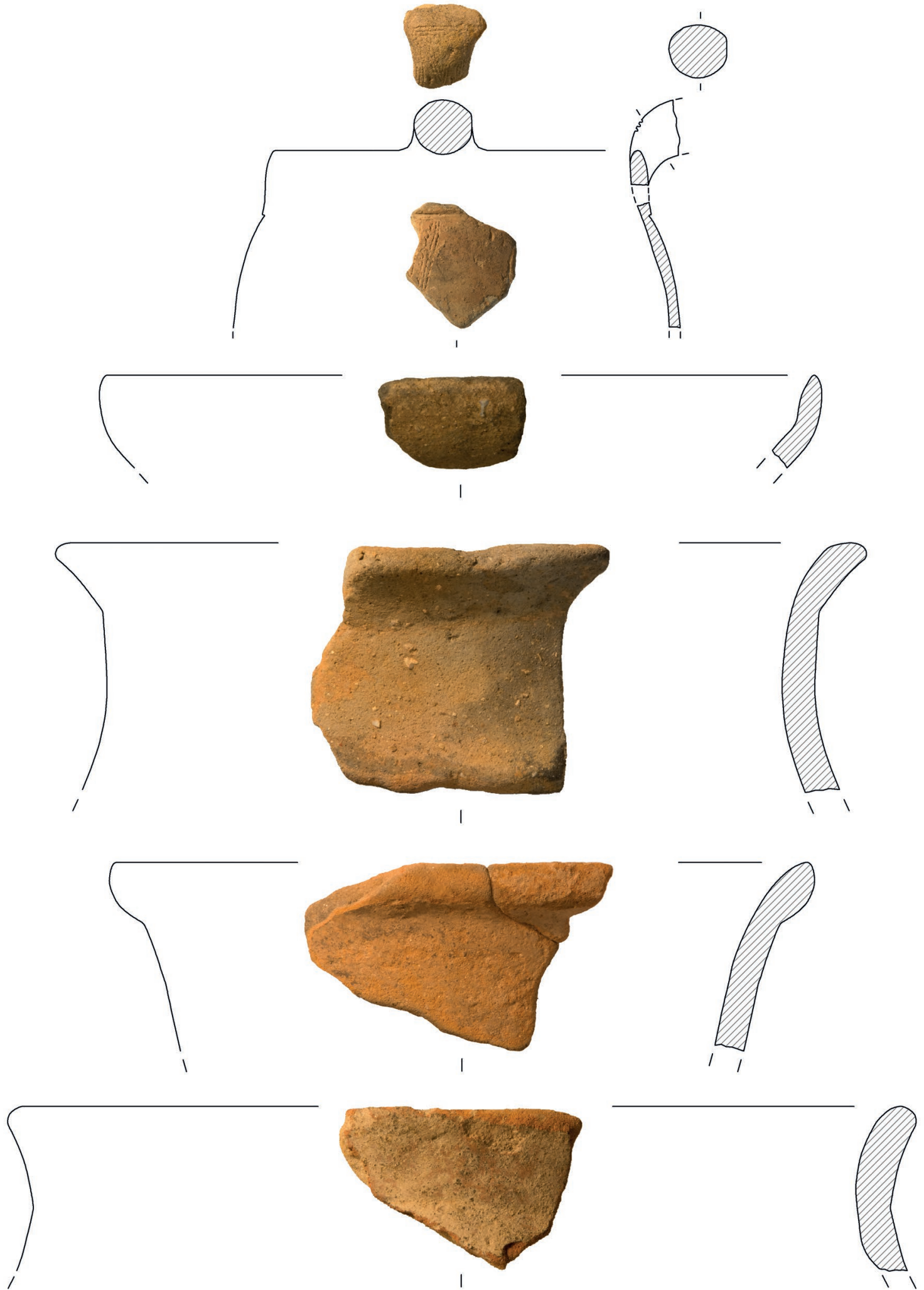

5

Tafel 11. Ada Tepe, Nordostquartier, Haus 7. Keramik. - 1. SE 09 (1:4). - 2-3. SE 10 (1:2). -4-5. SE 11 (1:2) (Zeichnungen: S. Eder, B. Horejs, St. Horvath, D. Kolic, S. Molla-Djafari, M. Röcklinger; Fotos: Th. Urban). 
Object ID: AT12/10/1/20

$\begin{array}{ll}\text { Shape: } & \text { base } \\ \text { Type: } & \text { flat base } \\ \text { Variant: } & \text { regular transition } \\ \text { Type code: } & \text { B2B } \\ \text { Preservation: } & \text { abraded } \\ \text { Fabric: } & 12 \\ \text { Dimensions (cm): } & \\ & \text { wall thickness: } 0.9 \\ & \text { diam. base: } 12 \\ & \text { diam. base, preserv. (\%): } 16\end{array}$

Object ID: AT12/10/1/21

Shape: base

Type: stepped base

Type code: B1

Preservation: abraded

Fabric: 3

Dimensions $(\mathrm{cm})$ :

wall thickness: 1.3

Object ID: AT12/10/1/16

Shape: base

Type: slightly stepped

Variant: curved

Type code: B4A

Preservation: abraded

Fabric: 7

Dimensions $(\mathrm{cm})$ : wall thickness: 1.2

\section{Vertical handle}

Object ID: AT12/10/1/23

Shape: vertical handle

Type: oval cross section

Variant: unclear position - horizontal

Type code: VH1B

Preservation: abraded

Fabric: 1

Dimensions (cm):

$$
\text { width: } 2.8
$$

thickness: 2.1

Object ID: AT12/10/1/24

Shape: vertical handle

Type: oval cross section

Variant: undecorated

Type code: VH1B

Preservation: abraded

Fabric: 12

Dimensions $(\mathrm{cm})$ :

width: 3.1

thickness: 2.7

Object ID: AT12/10/1/26

Shape: vertical handle

Type: oval cross section

Variant: undecorated

Type code: VH1B

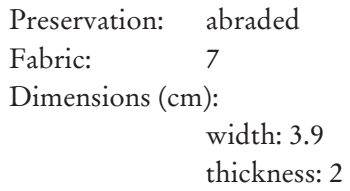

Handle-undefined

Object ID: AT12/10/1/22

Shape: handle-undefined

Type: oval cross section

Variant: undecorated

Type code: H1A

Preservation: abraded

Fabric: $\quad 7$

Dimensions (cm):

width: 3.6

thickness: 2.8

Object ID: AT12/10/1/28

Shape: handle-undefined

Type: oval cross section

Variant: undecorated

Type code: H1A

Preservation: abraded

Fabric: 1

Dimensions (cm):

width: 2.4

thickness: 2.3

Decorated wall fragment

Object ID: AT12/10/1/34

Shape: decorated wall fragment

Type: Furchenstich

Type code: DF9

Preservation: abraded

Fabric: $\quad 3$

Description: two parallel lines

Dimensions (cm):

wall thickness: 0.6

Object ID: AT12/10/1/30

Shape: decorated wall fragment

Type: stamped and grooved

Type code: DF1

Preservation: abraded

Fabric: $\quad 5$

Dimensions (cm):

wall thickness: 0.9 
Object ID: AT12/10/1/31

Shape: decorated wall fragment

Type: grooved

Variant: grooved

Type code: DF3

Fabric: $\quad 10$

Description: Three lines.

Dimensions (cm):

wall thickness: 0.8

Object ID: AT12/10/1/32

Shape: decorated wall fragment

Type: incised

Type code: DF2

Preservation: abraded

Fabric: $\quad 10$

Description: bundle of lines, possibly round shape; short lines outside, starting at outermost line, are possibly rays

Dimensions $(\mathrm{cm})$ : wall thickness: 0.6

\section{Plastic cordon}

Object ID: AT12/10/1/29

Shape: plastic cordon

Type: impressed

Variant: $\quad$ unclear position - horizontal

Type code: PC2A

Preservation: abraded

Fabric: $\quad 2$

Dimensions (cm):

diam. body: 60

diam. body, preserv. (\%): 7

wall thickness: 1.8

\section{Unit 11}

\section{Amphora}

Object ID: AT12/11/1/1+2 Tafel 11/4

Shape: amphora

Type: $\quad$ conical neck - bent outwards

Variant: bead rim

Type code: A2H

Preservation: abraded

Fabric: $\quad 12$

Dimensions (cm):

wall thickness: 0.9

diam. rim: 22

diam. rim, preserv. (\%): 10

\section{Deep bowl}

Object ID: AT12/11/1/3 Tafel 11/5

Shape: deep bowl

Type: funnel shaped neck

Variant: regular rounded lip

Type code: DB1A

Preservation: abraded

Fabric: $\quad 13$

Dimensions (cm):

wall thickness: 1.2

diam. rim: 28

diam. rim, preserv. (\%): 9

\section{Vertical handle}

Object ID: AT12/11/1/5

Shape: vertical handle

Type: $\quad$ round cross section

Variant: decorated

Type code: VH3B

Preservation: abraded

Fabric: $\quad 12$

Dimensions (cm):

$$
\text { width: } 1.9
$$

\section{Unit 12}

Beaker

$\begin{array}{lc}\text { Object ID: AT12/12/1/3 } & \text { Tafel } 12 / 1 \\ \text { Shape: } & \text { beaker }\end{array}$

Type: narrow mouthed

Variant: thinned lip

Type code: Be1C

Preservation: secondary burn

Fabric: $\quad 10$

Application: DF9

Description: triangular, leave-shaped motive and horizontal lines

Dimensions (cm):

wall thickness: 0.6

diam. rim: 6

diam. rim, preserv. $(\%): 18$

Decorated wall fragment

Object ID: AT12/12/1/1

Shape: decorated wall fragment

Type: $\quad$ Furchenstich

Type code: DF9

Preservation: secondary burn

Fabric: $\quad 12$

Description: bundle of lines (three lines) crossing the sherd three times; possibly zig-zag-band; not enough

Dimensions (cm): preserved

diam. body: 20

diam. body, preserv. (\%): 11

wall thickness: 0.8

Object ID: AT12/12/1/2

Shape: decorated wall fragment

Type: grooved

Type code: DF3

Preservation: secondary burn

Fabric: $\quad 9$

Description: bundle of four lines

Dimensions (cm):

diam. body: 15

wall thickness: 0.5

Object ID: AT12/12/1/4 Tafel 12/2

Shape: decorated wall fragment

Type: incised

Type code: DF2

Preservation: abraded

Fabric: 6

Description: s-shaped hook, horizontal lines beneath and above of it 
Dimensions (cm):

diam. body: 26

wall thickness: 0.7

\section{Unit 13}

Irregular shape

Object ID: AT13/13/1/9

Shape: irregular shape

Fabric: 1

Dimensions (cm):

width: 5.5

\section{Amphora}

Object ID: AT13/13/1/1

Shape: amphora

Type: conical neck - bent outwards

Variant: regular rounded lip

Type code: A2A

Preservation: abraded

Fabric:

Dimensions (cm):

wall thickness: 1.1

Object ID: AT13/13/1/4

Shape: amphora

Type: $\quad$ conical neck - bent outwards

Variant: thinned lip

Type code: A2C

Preservation: abraded

Fabric: 13

Dimensions (cm):

wall thickness: 0.6

diam. rim: 10

diam. rim, preserv. (\%): 16

\section{Jar}

Object ID: AT13/13/1/2

Shape: jar

Type: not definable

Variant: regular rounded lip

Type code: JOA

Preservation: secondary burn

Fabric: $\quad 11$

Application: PC1A

Description: angular finger impressions

Dimensions (cm):

wall thickness: 0.9

\section{Shallow bowl}

Object ID: AT13/13/1/14 Tafel 12/4

Shape: shallow bowl

Type: slightly inverted rim

Variant: thinned lip

Type code: $\quad$ SB2C

Preservation: abraded

Fabric: 4

Application: DF9

Description: rhombic motive; Connected rhombi

Dimensions (cm):
Lid

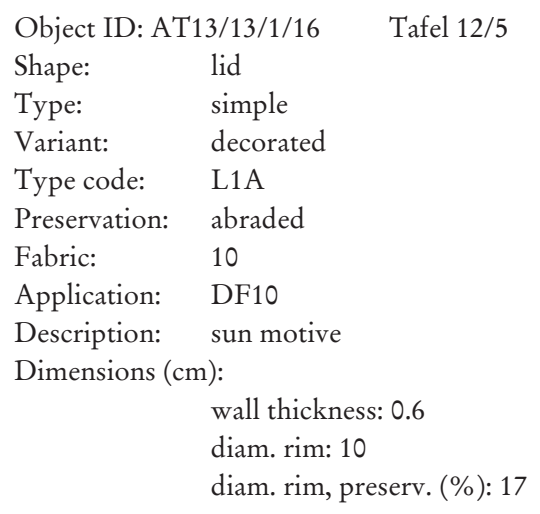

Vertical handle

Object ID: AT13/13/1/11

Shape: vertical handle

Type: oval cross section

Variant: undecorated

Type code: VH1B

Preservation: abraded

Fabric: $\quad 3$

Dimensions $(\mathrm{cm})$ :

width: 4.3

thickness: 1.8

Object ID: AT13/13/1/7

Shape: vertical handle

Type: oval cross section

Variant: undecorated

Type code: VH1B

Preservation: abraded

Fabric: 7

Dimensions (cm):

width: 4.4

thickness: 3.2

Object ID: AT13/13/1/8

Shape: vertical handle

Type: oval cross section

Variant: undecorated

Type code: VH1B

Preservation: abraded

Fabric: 12

Dimensions (cm):

width: 2.5

thickness: 2.1

Horizontal bandle

Object ID: AT13/13/1/5

Shape: horizontal handle

Type: oval cross section

Variant: undecorated

Type code: HH1A

Preservation: secondary burn

Fabric: 7

Description: slagged 

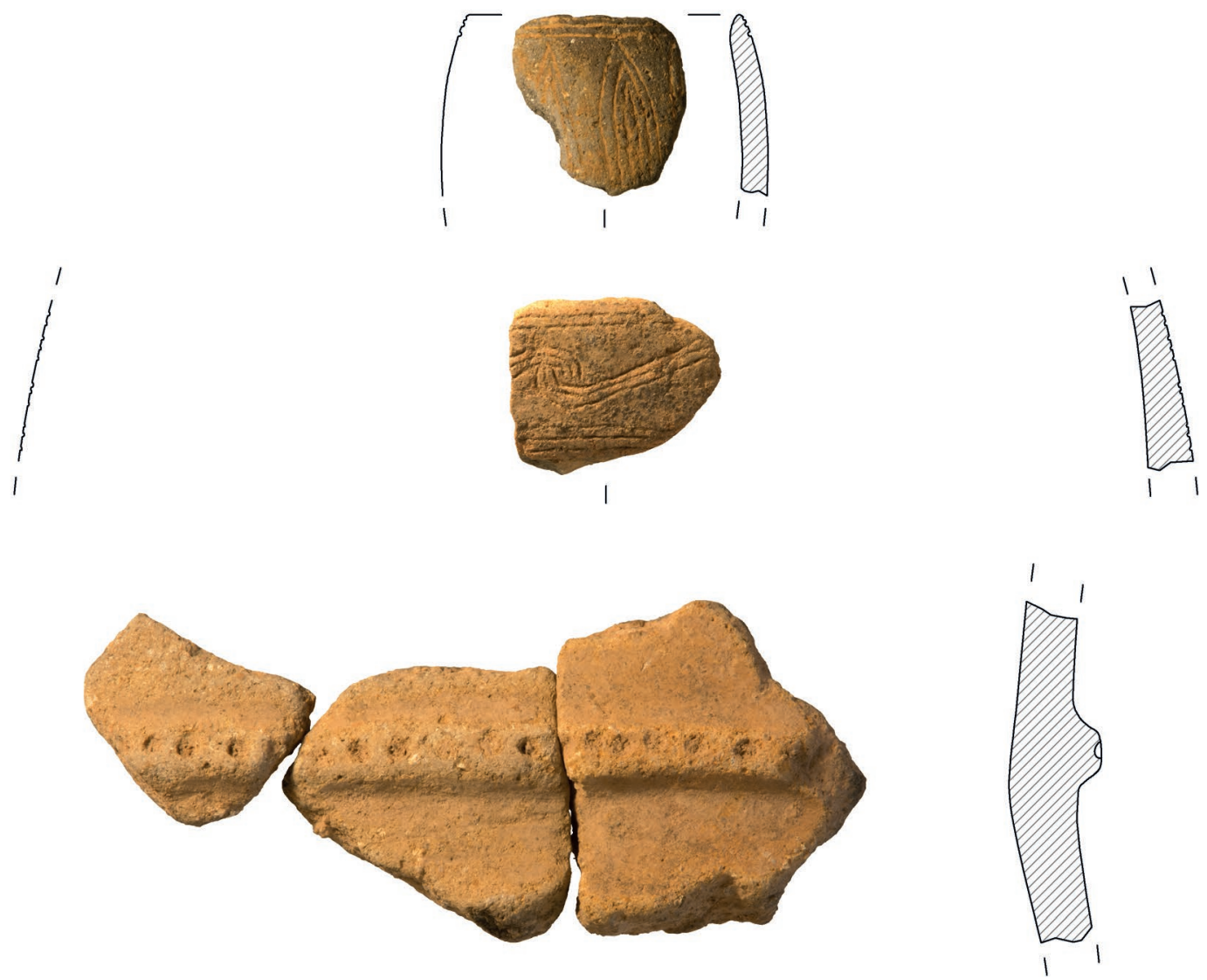

4
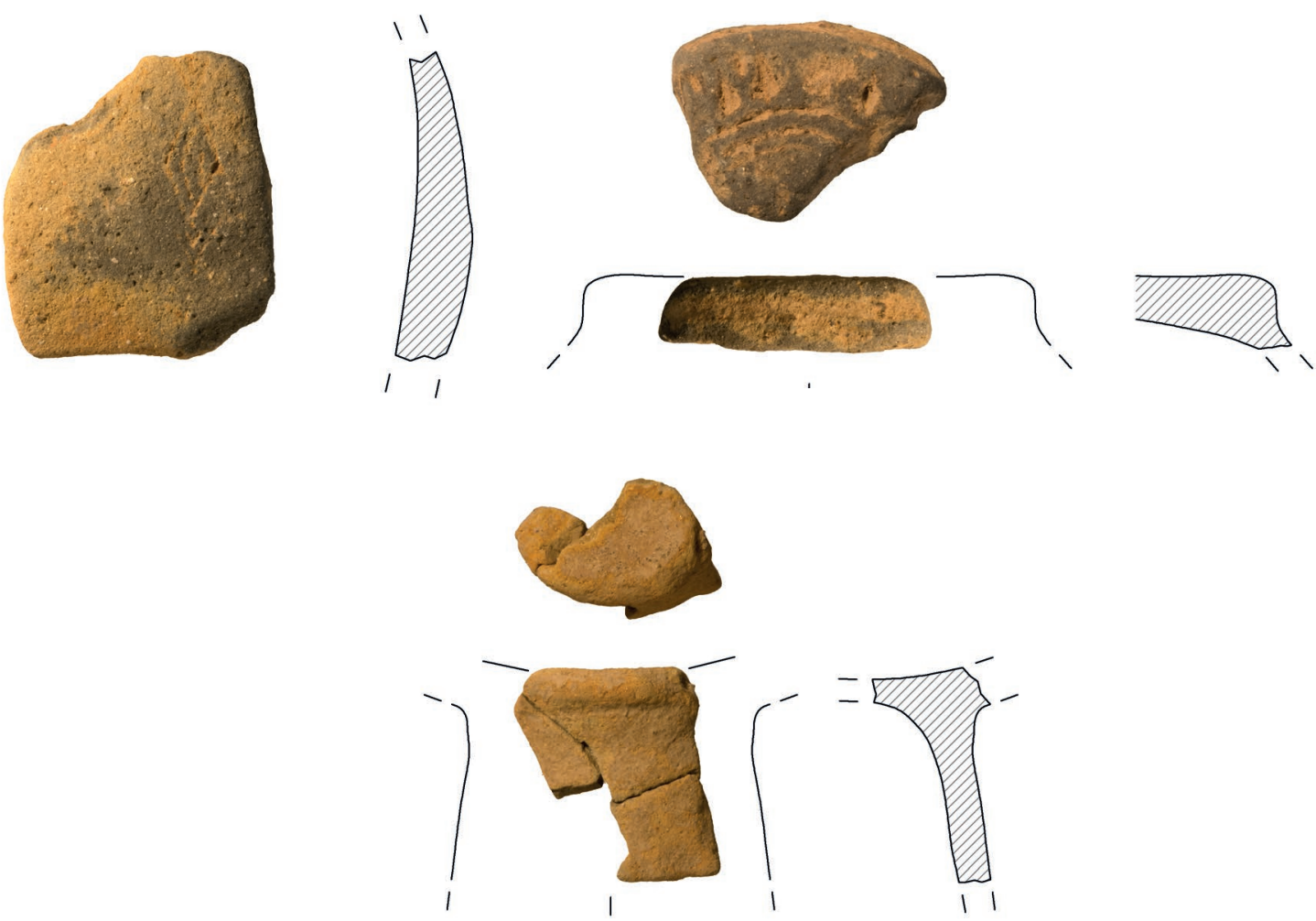

Tafel 12. Ada Tepe, Nordostquartier, Haus 7. Keramik. -1-2. SE 12 (1:2). - 3-5. SE 13 (1:2). - 6. SE 16 (1:2) (Zeichnungen: S. Eder, B. Horejs, St. Horvath, D. Kolic, S. Molla-Djafari, M. Röcklinger; Fotos: Th. Urban). 
Dimensions (cm):

width: 3.1

thickness: 2.9

\section{Handle - undefined}

Object ID: AT13/13/1/6

Shape: handle-undefined

Type: oval cross section

Variant: undecorated

Type code: H1A

Preservation: abraded

Fabric: $\quad 7$

Dimensions $(\mathrm{cm})$ :

width: 3.4

thickness: 2.7

\section{Decorated wall fragment}

Object ID: AT13/13/1/15

Shape: decorated wall fragment

Type: incised and Furchenstich

Type code: DF11

Preservation: abraded

Fabric: $\quad 6$

Description: bundle of lines (four lines), incised and Furchenstich, running oblique to an angle; lined by cut-out triangles

Dimensions $(\mathrm{cm})$ : wall thickness: 1

Plastic cordon

Object ID: AT13/13/1/13 Tafel 12/3

Shape: plastic cordon

Type: impressed

Variant: unclear position - horizontal

Type code: PC2A

Preservation: abraded

Fabric: $\quad 4$

Dimensions (cm):

wall thickness: 1.4

Object ID: AT13/13/1/12

Shape: plastic cordon

Type code: $\quad$ PC2

Preservation: abraded

Fabric: $\quad 6$

Application: PC2

Description: cordon possibly attached to transition of neck and shoulder

Dimensions $(\mathrm{cm})$ :

wall thickness: 0.9

\section{Unit 14}

Amphora

Object ID: AT12/14/1/1

Shape: amphora

Type: $\quad$ cylindrical neck - conical

Variant: regular rounded lip

Type code: A1A

Preservation: abraded

Fabric:
Dimensions $(\mathrm{cm})$ :

wall thickness: 0.9

diam. rim: 14

diam. rim, preserv. (\%): 6

Base

Object ID: AT12/14/1/4

Shape: base

Type: slightly stepped

Variant: curved

Type code: B4A

Preservation: abraded

Fabric: $\quad 3$

Dimensions $(\mathrm{cm})$ :

wall thickness: 1.2

diam. base: 10

diam. base, preserv. (\%): 15

Object ID: AT12/14/1/3

Shape: base

Type: flat base

Variant: regular transition

Type code: B2B

Preservation: abraded

Fabric: $\quad 3$

Dimensions $(\mathrm{cm})$ :

wall thickness: 1.5

Object ID: AT12/14/1/2

Shape: base

Type: slightly stepped

Variant: curved

Type code: B4A

Preservation: abraded

Fabric: $\quad 7$

Dimensions $(\mathrm{cm})$ :

wall thickness: 1.3

\section{Vertical bandle}

Object ID: AT12/14/1/6

Shape: vertical handle

Type: oval cross section

Variant: undecorated

Type code: VH1B

Fabric: $\quad 6$

Description: construction plug

Dimensions $(\mathrm{cm})$ :

width: 2.5

thickness: 1.5

Object ID: AT12/14/1/5

Shape: vertical handle

Type: oval cross section

Variant: undecorated

Type code: VH1B

Preservation: abraded

Fabric: $\quad 1$

Dimensions (cm):

width: 3.6

thickness: 1.8 
Unit 15

Amphora

Object ID: AT12/15/1/1

Shape: amphora

Type: $\quad$ cylindrical neck - conical

Variant: $\quad$ regular rounded lip

Type code: A1A

Preservation: abraded

Fabric: $\quad 13$

Dimensions $(\mathrm{cm})$ :

wall thickness: 0.8

diam. rim: 16

diam. rim, preserv. (\%): 14

\section{Shallow bowl}

Object ID: AT12/15/1/4

Shape: shallow bowl

Type: s-shaped

Variant: regular rounded lip

Type code: SB1A

Preservation: abraded

Fabric: $\quad 4$

Dimensions (cm):

wall thickness: 0.7

\section{Base}

Object ID: AT12/15/1/5

Shape: base

Type: slightly stepped

Variant: curved

Type code: $\quad$ B4A

Preservation: abraded

Fabric: $\quad 1$

Dimensions (cm):

wall thickness: 1.3

\section{Handle - undefined}

Object ID: AT12/15/1/6

Shape: handle - undefined

Type: $\quad$ round cross section

Variant: undecorated

Type code: H2A

Preservation: abraded

Fabric: 12

Dimensions (cm):

width: 1.6

Decorated wall fragment

Object ID: AT12/15/1/7

Shape: decorated wall fragment

Type: incised

Type code: DF2

Preservation: abraded

Fabric: 9

Description: two parallel lines

Dimensions (cm):

wall thickness: 0.7

\section{Unit 16}

Jar

Object ID: AT12/16/1/1

Shape: jar

Type: $\quad$ narrow mouthed - conical

Variant: regular rounded lip

Type code: J2A

Preservation: abraded

Fabric: $\quad 1$

Dimensions (cm):

wall thickness: 2.1

Base

Object ID: AT12/16/1/3 Tafel 12/6

Shape: base

Type: ring base

Variant: straight

Type code: B5B

Preservation: abraded

Fabric: $\quad 13$

Description: surface partially medium burnished

Dimensions (cm):

wall thickness: 0.5

diam. base: 7

diam. base, preserv. (\%): 12

\section{Vertical handle}

Object ID: AT12/16/1/4

Shape: vertical handle

Type: oval cross section

Variant: undecorated

Type code: VH1A

Preservation: abraded

Fabric: $\quad 1$

Dimensions (cm):

width: 5.1

thickness: 3

Object ID: AT12/16/1/6

Shape: vertical handle

Type: oval cross section

Variant: undecorated

Type code: VH1B

Preservation: abraded

Fabric: $\quad 7$

Dimensions (cm):

width: 2.9

thickness: 2

Object ID: AT12/16/1/5

Shape: vertical handle

Type: oval cross section

Variant: undecorated

Type code: VH1B

Preservation: abraded

Fabric: 2

Dimensions (cm):

width: 3.1

thickness: 2.6 

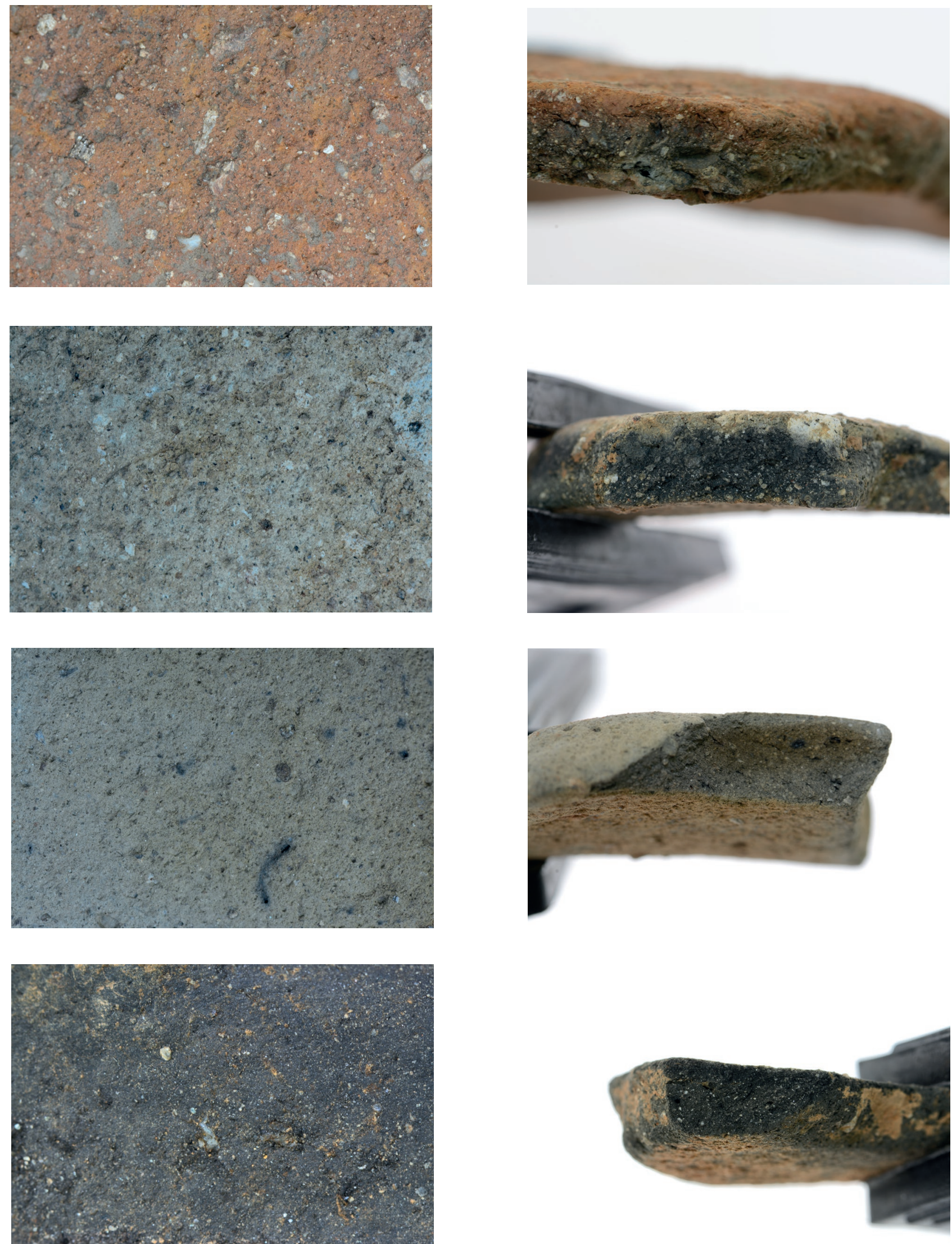

Tafel 13. Ada Tepe, Nordostquartier, Haus 7. Warengruppen 1, 4, 5 und 10 (Fotos: F. Ostmann). 

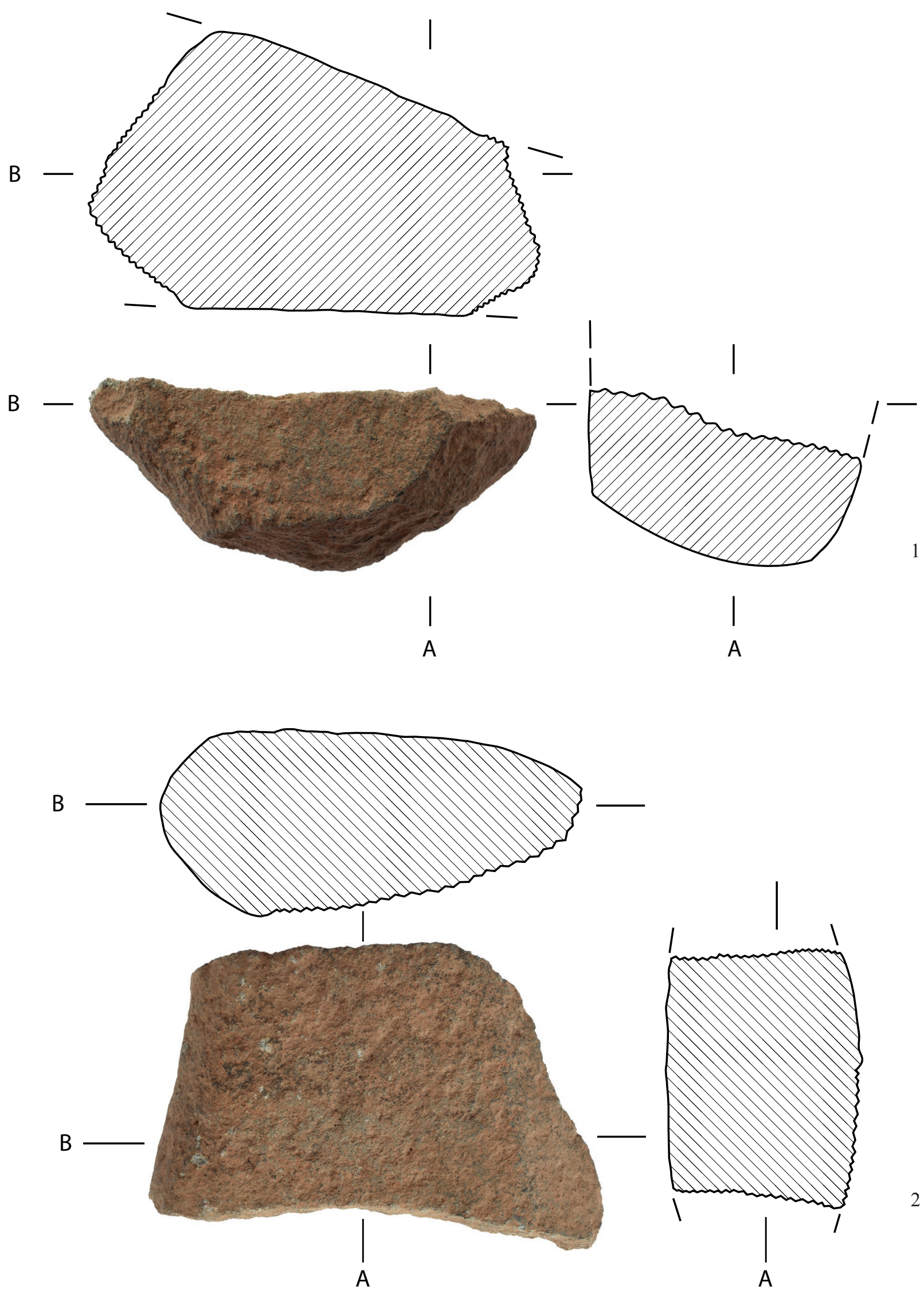

Tafel 14. Ada Tepe, Nordostquartier, Haus 7. Reibsteine AT13/170/7/1 und AT12/172/7/1 (1:2) (Zeichnungen: D. Blattner, S. Eder, S. Molla-Djafari, F. Ostrowski, M. Röcklinger; Fotos: F. Ostmann). 


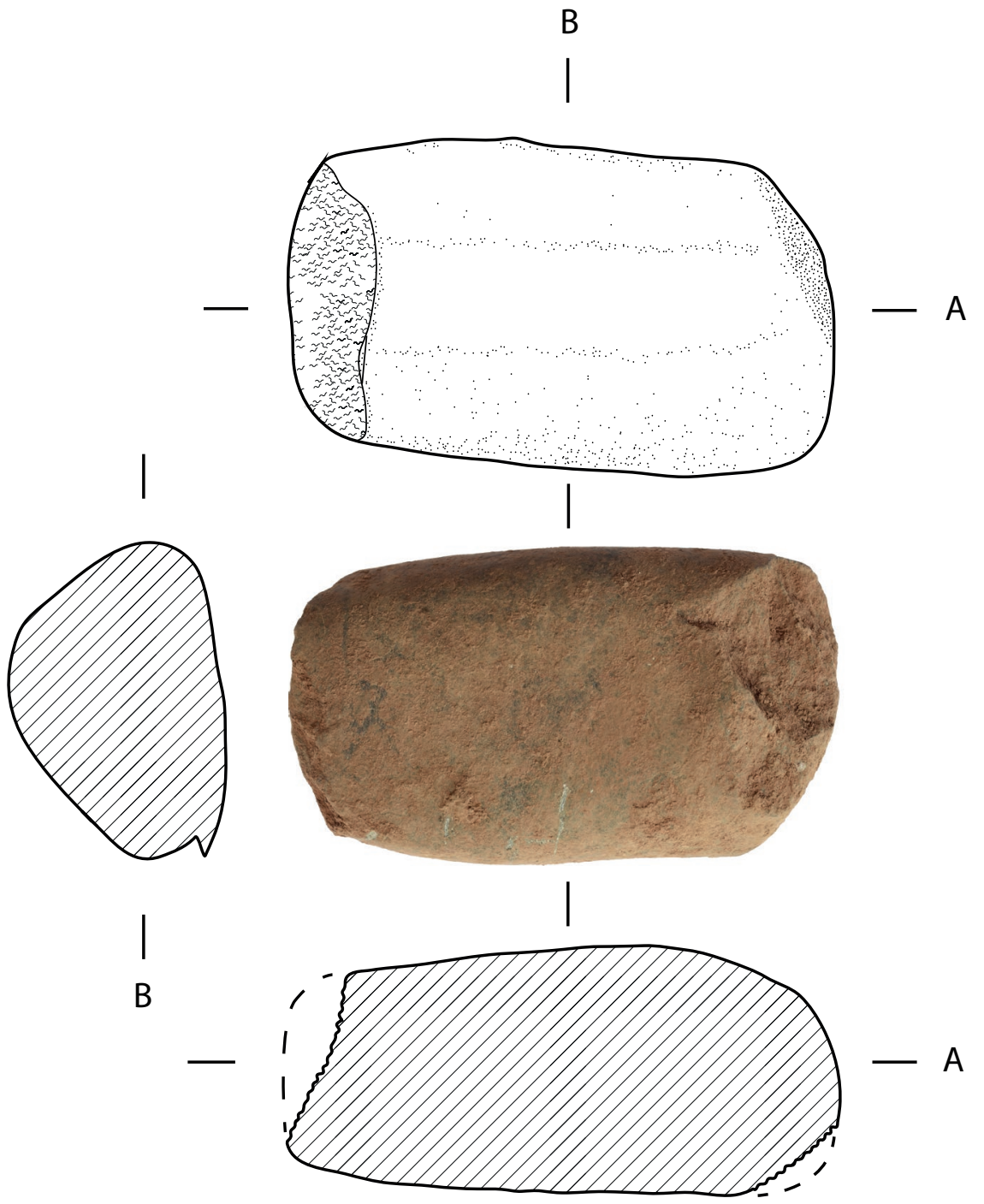

Tafel 15. Ada Tepe, Nordostquartier, Haus 7. Reibstein AT13/174/7/1 (1:2) (Zeichnungen: D. Blattner, S. Eder, S. Molla-Djafari, F. Ostrowski, M. Röcklinger; Fotos: F. Ostmann). 


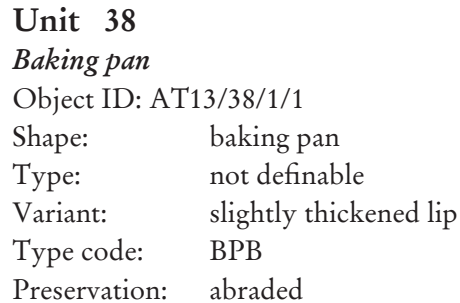

\subsection{Grinding stones}

Object ID: AT13/170/7/1 Tafel 14/1

Description: fragment of a lower part of a grinding stone; grinding surface partially preserved, plan

Material: $\quad$ stone type unknown

Dimensions (cm):

length: 15.5

width: 9.8

hight: 5.10

Object ID: AT12/172/7/1 Tafel 14/2

Description: fragment of an upper part of a grinding stone; elongated shape, broken on both sides; heavily rounded-triangular cross section with one steep side; grinding surface slightly concave in cross section and approximately plan in longitudinal section

Material: $\quad$ stone type unknown

Dimensions (cm):

length: 14.2

width: 9.3

height: 7

Object ID: AT13/174/7/1 Tafel 15/1

Description: upper part of a grinding stone; elongated-oval shape; rounded-triangular cross section; grinding surface concave in cross section and slightly concave in longitudinal section; broken on one end

Material: $\quad$ stone type unknown

Dimensions (cm):

length: 13

width: 7.9

hight: 5-5.9

\section{Danksagung}

Mein herzlicher Dank gilt in erster Linie dem Leiter des Ada Tepe Projekts, H. Popov sowie dem Nationalen Archäologischen Institut mit Museum (NAIM) der Bulgarischen Akademie der Wissenschaften für die Einladung in dieses Projekt und die Zurverfügungstellung der Daten und Funde. Des Weiteren danke ich Th. Urban für die Erstellung der Digitalzeichnungen auf den Tafeln sowie M. Röcklinger, S. Molla-Djafari, D. Blattner und L. Burkhardt für das Prozessieren und die Überarbeitung der primären Daten und die Erstellung von Grafiken, Katalog und statistischer Auswertung. G. Ruß-Popa danke ich für wertvolle Hinweise zur Bergwerksforschung in Österreich und Iran; R. Jung danke ich für konstruktive Diskussionen zur möglichen Rolle der Goldproduzenten. Schließlich gilt mein herzlicher Dank dem gesamten Ada Tepe Team und ganz besonders Antoaneta
Andonova, Zornitsa Krasteva und Marina Koleva für die geduldige Hilfe im Depot von Krumovgrad. Die Finanzierung verdanken wir dem Österreichischen Wissenschaftsfond (FWF Projekt Nr. P 28451).

\section{Literatur}

Aali, StÖllner 2015

A. AALI, Th. STÖLLnER, The archaeology of salt miners: interdisciplinary research 2010-2014. Metalla 21/1-2, Bochum 2014.

Alexandrov 2002

St. Alexandrov, The Late Bronze Age settlement at Koprivlen. In: A. Bozkova, P. Detev (Hrsg.), Koprivlen 1: Rescue Archaeological Investigations along the Gotse Delchev - Drama Road 1998-1999. Sofia 2002, 63-82, 323-340, 453-454.

AleXANDRov 2017

St. Alexandrov, Der Grabhügel-Komplex (Grabstätte Nr. 7) von Ovčarci. In: S. HaAg, Ch. Popov, B. Horejs, St. Alexandrov, G. Plattner (Hrsg.), Das erste Gold: Ada Tepe: Das älteste Goldbergwerk Europas. Ausstellungskatalog der Sonderausstellung im KHM 2017, Wien 2017, 130-132.

Alexandrov, Christova, Marinov 2017

St. Alexandrov, T. Christova, M. Marinov, Der Hortfund von Svištov. In: S. HaAg, Ch. Popov, B. Horejs, St. Alexandrov, G. Plattner (Hrsg.), Das erste Gold: Ada Tepe: Das älteste Goldbergwerk Europas. Ausstellungskatalog der Sonderausstellung im KHM 2017, Wien 2017, 133-136.

Andreou, Psaraki 2007

St. Andreou, K. Psaraki, Tradition and innovation in the Bronze Age pottery of the Thessaloniki Toumba: food and drink consumption and "tableware" ceramics. In: H. Todorova, M. STEfanovich, G. Ivanov (Hrsg.), The Struma/Strymon River Valley in Prehistory. Proceedings of the International Symposium „Strymon Praehistoricus“, Kjustendil-Blagoevgrad-SerresAmphipolis, 27.09-01.10.2004. In The Steps of James Harvey Gaul 2, Sofia 2007, 397-420.

Andreou et al. 2013

St. Andreou, C. Heron, J. Glynis, V. Kiriatzi, K. Psaraki, M. Roumpou, S. M. Valamoti, Smelly barbarians or perfumed natives? An investigation of oil and ointment use in Late Bronze Age northern Greece. In: S. Voutsaki, S. M. Valamoti (Hrsg.), Diet, Economy and Society in the Ancient Greek World: Towards a Better Integration of Archaeology and Science. Proceedings of the International Conference held at the Netherlands Institute at Athens on 22-24 March 2010. Pharos Supplement 1, Leuven - Paris - Walpole 2013, 173-185.

Athanassov 2011

B. Athanassov, Siedlungsmobilität im zweiten Jahrtausend v. Chr. auf dem Ostbalkan. In: V. Nikolov, K. Bacvarov, H. Popov (Hrsg.), Interdisziplinäre Forschungen zum Kulturerbe auf der Balkanhalbinsel. Beiträge des Humboldt-Kollegs Interdisziplinäre Forschungen zum Kulturerbe auf der Balkanhalbinsel, 19.-22. November 2009, Sofia, Bulgarien. Sofia 2011, 177-208.

Athanassov, Krauss 2015

B. Athanassov, R. Krauss, Der Ostbalkanraum zwischen mediterranen Hochkulturen und dem südöstlichen Europa. In: G. voN BüLOw (Hrsg.), Kontaktzone Balkan. Beiträge des internationalen Kolloquiums „Die Donau-Balkan-Region als Kontaktzone zwischen Ost-West und Nord-Süd“ vom 16.-18. Mai 2012 in Frankfurt a. M. Kolloquien zur Vor- und Frühgeschichte 20, Bonn 2015, 63-79. 
Athanassov, Krauss, Slavčev 2009

B. Athanassov, R. Krauss, V. SlavčEv, Ein Bronzeschwert ägäischanatolischen Typs aus dem Museum von Varna, Bulgarien, Analele Banatului 17, 2009, 17-32.

Bozhinova, Jung, Mommsen 2013

E. Bozhinova, R. Jung, H. Mommsen, Dragojna - eine spätbronzezeitliche Höhensiedlung in den bulgarischen Rhodopen mit importierter mykenischer Keramik, Mitteilungen des Deutschen Archäologischen Instituts Athenische Abteilung 125/2010, 2013, $45-97$.

BorisLaVov 2017

B. Borislavov, Grabhügel 1 von Izvorovo. In: S. HaAg, Ch. Popov, B. Horejs, St. Alexandrov, G. Plattner (Hrsg.), Das erste Gold: Ada Tepe: Das älteste Goldbergwerk Europas. Ausstellungskatalog der Sonderausstellung im KHM 2017, Wien 2017, $128-129$.

Dimitrova 2014

Я. Аимитрова, Керамичен комплекс от късната бронзова и ранната желязна епоха от светилището Бабяк, Археология 40, 1-2, 2014, $7-27$.

FischL, KISS, KULCSÁR 2001

K. Fischl, V. Kiss, G. Kulcsár, Beiträge zum Gebrauch der tragbaren Feuerherde (Pyraunoi) im Karpatenbecken II (Spätbronzezeit - Früheisenzeit). In: C. KACsó (Hrsg.), Der nordkarpatische Raum in der Bronzezeit. Symposium Baia Mare , 7.-10. Oktober 1998. Baia Mare 2001, 125-156.

Grammenos 1981

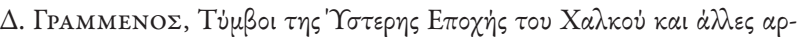

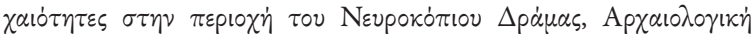

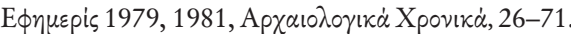

GRÖMER 2010

K. Grömer, Prähistorische Textilkunst in Mitteleuropa - Geschichte des Handwerks und der Kleidung vor den Römern. Veröffentlichungen der Prähistorischen Abteilung des Naturhistorischen Museums 4, Wien 2010.

HAAG et al. 2017

S. Haag, Ch. Popov, B. Horejs, St. Alexandrov, G. Plattrner (Hrsg.), Das erste Gold: Ada Tepe: Das älteste Goldbergwerk Europas. Ausstellungskatalog der Sonderausstellung im KHM 2017, Wien 2017.

HäNSEL 1976

B. HäNSEL, Beiträge zur regionalen und chronologischen Gliederung der älteren Hallstattzeit an der unteren Donau. Beiträge zur urund frühgeschichtlichen Archäologie des Mittelmeer-Kulturraumes 16, Bonn 1976.

HäNSEL 1982

B. HäNSEL, Südosteuropa zwischen 1600 und 1000 v. Chr. In: B. HäNSEL (Hrsg.), Südosteuropa zwischen 1600 und 1000 v. Chr. Prähistorische Archäologie in Südosteuropa 1, Berlin 1982, 1-38.

HOCHSTETTER 1984

A. HochstetTer, Kastanas. Ausgrabungen in einem Siedlungshügel der Bronze- und Eisenzeit Makedoniens 1975-1979: Die Handgemachte Keramik, Schichten 19 bis 1. Prähistorische Archäologie in Südosteuropa 3, Berlin 1984.

HOREJS 2005

B. Horejs, Kochen am Schnittpunkt der Kulturen - zwischen Karpatenbecken und Ägäis. In: B. Horejs, R. Jung, E. KaiseR, B. TeržAn (Hrsg.), Interpretationsraum Bronzezeit. Bernhard Hänsel von seinen Schülern gewidmet. Universitätsforschungen zur prähistorischen Archäologie 121, Bonn 2005, 71-94.
HOREJS 2007a

B. Horejs, Das Prähistorische Olynth. Ausgrabungen in der Toumba Agios Mamas 1994-1996: Die spätbronzezeitliche handgemachte Keramik der Schichten 13 bis 1. Prähistorische Archäologie in Südosteuropa 21, Rahden/Westf. 2007.

Horejs 2007b

B. Horejs, Macedonia: mediator or buffer zone between cultural spheres? In: I. Galanaki, H. Tomas, Y. Galanakis, R. LaffiNeur (Hrsg.), Between the Aegean and Baltic Seas: Prehistory Across Borders. Proceedings of the International Conference Bronze and Early Iron Age Interconnections and Contemporary Developments between the Aegean and the Regions of the Balkan Peninsula, Central and Northern Europe. University of Zagreb, 11-14 April 2005. Aegaeum 27, Eupen 2007, 293-306.

HOREJS 2009

B. Horejs, Alltagskulturen und Eliten zwischen Karpatenbecken und Ägäis: verschiedene Kommunikationsmodelle? In: A. Kreen-Leeb, H.-J. Beier, E. Classen, F. Falkenstein, St. Schwenzer (Hrsg.), Mobilität, Migration und Kommunikation in Europa während des Neolithikums und der Bronzezeit. Beiträge der Sitzungen der Arbeitsgemeinschaften Neolithikum und Bronzezeit während der Jahrestagung des West- und Süddeutschen Verbandes für Altertumsforschung e. V. in Xanten, 6.-8. Juni 2006. Varia Neolithica V, Beiträge zur Ur- und Frühgeschichte Mitteleuropas 53, Langenweissbach 2009, 197-207.

Horejs 2010

B. Horejs, Possibilities and limitations in analysing ceramic wares. In: B. Horejs, R. Jung, P. Pavúk (Hrsg.), Analysing Pottery: Processing, Classification, Publication. Bratislava 2010.

Jones et al. 1986

G. Jones, K. Wardle, P. Halstead, D. Wardle, Crop storage at Assiros, Scientific American 254, 3, 1986, 96-103.

Jung 2017

R. Jung, Krieger und Waffen auf dem Zentral- und Ostbalkan. In: S. Haag, Ch. Popov, B. Horejs, St. Alexandrov, G. Plattner (Hrsg.), Das erste Gold: Ada Tepe: Das älteste Goldbergwerk Europas. Ausstellungskatalog der Sonderausstellung im KHM 2017, Wien 2017, 69-73.

JUNG et al. 2017

R. Jung, St. Alexandrov, E. Bozhinova, H. Mommsen, Mykenische Keramik aus der Rhodopenregion. Herkunft, regionaler Kontext und sozialökonomische Grundlagen, Archaeologia Austriaca 101, 2017, 269-302.

KERN et al. 2008

A. Kern, K. Kowarik, A. W. Rausch, H. Reschreiter (Hrsg.), Salz-Reich: 7000 Jahre Hallstatt. Veröffentlichungen der Prähistorischen Abteilung 2, Wien 2008.

KISJOv 1994

К. Кисьов, Керамиката от късната бронзова епоха в гробните компмекси от централните родопи, Археология XXXV/2, 1994, $1-13$.

Koukouli-Chrysanthaki 1992

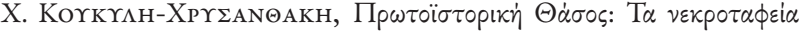

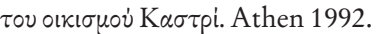

LESHTAKOV 2008

K. Leshtakov, New evidence on the LBA mortuary practices in south Bulgaria. In: O. Özbek (Hrsg.), Funeral Rites, Rituals and Ceremonies from Prehistory to Antiquity. Istanbul 2008, 69-82. 
LESHTAKOV 2009

K. Leshtakov, The second millenium BC in the northern Aegean and the adjacent Balkan lands: main dynamics of cultural interac-

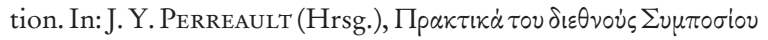

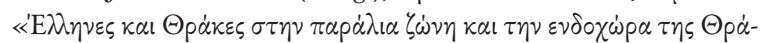

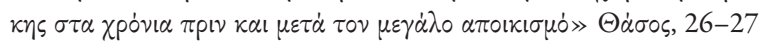
$\sum \varepsilon \pi \tau \varepsilon \mu \beta$ piov 2009. Acts of the international Symposium "Greeks and Thracians along the coast and in the Hinterland of Thrace during the years before and after the great colonization”, Thasos, 26-27 September 2009, Thasos 2009, 53-82.

Margomenou 2008

D. Margomenou, Food storage in prehistoric northern Greece: interrogating complexity at the margins of the "Mycenaean world", Journal of Mediterranean Archaeology 21/2, 2008, 191-212.

Nechrizov 1995

G. Nechrizov, Late Bronze Age pottery in the eastern Rhodopes. In: S. Alexandrov, D. W. Bailey, I. Panayatov (Hrsg.), Prehistoric Bulgaria. Monographs in World Archaeology 22, Madison, Wisconsin 1995, 309-325.

Nechrizov, CvetKova 2017

G. Nechrizov, J. Cvetrova, Die Bronzezeit in den östlichen Rhodopen. In: S. Haag, Ch. Popov, B. Horejs, St. AlexanDrov, G. Plattner (Hrsg.), Das erste Gold: Ada Tepe: Das älteste Goldbergwerk Europas. Ausstellungskatalog der Sonderausstellung im KHM 2017, Wien 2017, 49-55.

Nikov 2017

K. Nikov, Die Keramik vom Ada Tepe im Kontext der spätbronzezeitlichen Keramik in Thrakien. In: S. HaAg, Ch. Popov, B. Horejs, St. Alexandrov, G. Plattner (Hrsg.), Das erste Gold: Ada Tepe: Das älteste Goldbergwerk Europas. Ausstellungskatalog der Sonderausstellung im KHM 2017, Wien 2017, 63-67.

PANY 2008

D. Pany, Die Bevölkerung des Hallstätter Hochtales in der Älteren Eisenzeit. In: A. Kern, K. Kowarik, A. W. Rausch, H. Reschreiter (Hrsg.), Salz-Reich: 7000 Jahre Hallstatt. Veröffentlichungen der Prähistorischen Abteilung 2, Wien 2008, 136-141.

Popov 2009

Х. Попов, Куш кая: Характеристика на обитаването през късната бронзова и ранната жемязна епоха, Археология L, 1/2, 2009, 21-39.

Popov 2016

H. Popov, Kush Kaya on the absolute chronology of the Late Bronze Age in southern Thrace. In: K. Bacvarov, R. Gleser (Hrsg.), Southeast Europe and Anatolia in Prehistory: Essays in Honor of Vassil Nikolov on his $65^{\text {th }}$ anniversary. Universitätsforschungen zur prähistorischen Archäologie 293, Bonn 2016, 429-437.

Popov, JocKenHÖVEl 2011

H. Popov, A. Jockenhövel, At the northern borders of the Mycenean world: Thracian gold mining from the Late Bronze and the Early Iron Age at Ada Tepe in the eastern Rhodopes, Anodus: Studies of the Ancient World 10/2010, 2011, 265-281.

Popov, Nikov im Druck

H. Popov, K. Nikov, 'Ada Tepe Late Bronze Age Gold Mine’ project: between borders. In: S. Gimatzidis, M. Pieniążek, S. ManGaloĞlu-Votruba (Hrsg.), Archaeology across Frontiers and Borderlands: Fragmentation and Connectivity in the North Aegean and the Central Balkans from the Bronze Age to the Iron Age. Oriental and European Archaeology, Wien, im Druck.
Popov, Jockenhövel, Groer 2011

H. Popov, A. Jockenhövel, C. Groer, Ada Tepe (Ost-Rhodopen, Bulgarien): Spätbronzezeitlicher - ältereisenzeitlicher Goldbergbau, Kampagne 2008. In: Ü. YALçıN (Hrsg.), Anatolian Metal V, Der Anschnitt Beiheft 24. Veröffentlichungen aus dem Deutschen Bergbau-Museum 180, Bochum 2011, 111-126.

Popov, Nikov, Jockenhövel 2015

H. Popov, K. Nikov, A. Jockenhövel, Ada Tepe (Krumovgrad, Bulgarien) - ein neu entdecktes spätbronzezeitliches Goldbergwerk im balkanisch-ägäischen Kommunikationsnetz. In: G. voN BüLOw (Hrsg.), Kontaktzone Balkan. Beiträge des internationalen Kolloquiums „Die Donau-Balkan-Region als Kontaktzone zwischen Ost-West und Nord-Süd“ vom 16.-18. Mai 2012 in Frankfurt a. M. Kolloquien zur Vor- und Frühgeschichte 20, Bonn 2015, 45-62.

Popov et al. 2011

H. Popov, A. Jockenhövel, Z. Tsintsov, S. Iliev, Monatanarchäologische Forschungen in den Ostrhodopen, Südostbulgarien. In: V. Nikolov, K. E. Băčvarov, H. Popov (Hrsg.), Interdisziplinäre Forschungen zum Kulturerbe auf der Balkanhalbinsel. Beiträge des Humboldt-Kollegs Interdisziplinäre Forschungen zum Kulturerbe auf der Balkanhalbinsel, Sofia 19.- 22. November 2009, Sofia 2011, 253-290.

Popov et al. 2014

H. Popov, Z. Tsintsov, A. Jockenhövel, P. Georgiev, Feuersetzen beim Abbau der goldhaltigen Quarzgänge im spätbronzezeitlichen Goldbergwerk auf dem Ada Tepe, Südbulgarien, Experimentelle Archäologie in Europa 13, 2014, 27-44.

Popov et al. 2017

H. Popov, M. Koleva, A. Andonova, J. Dimitrova, I. Vălčev, Das Goldbergwerk auf dem Ada Tepe: Zu Topografie, Stratigrafie, Chronologie und Interpretation des Nordostquartiers, Archaeologia Austriaca 101, 2017, 161-204.

Popov et al. im Druck

H. Popov, E. Marinova, I. Hristova, St. Iliev, Plant food from the Late Bronze Age and Iron Age hilltop site Kush Kaya, Eastern Rhodope Mountains, Bulgaria: insights on the cooking practices. In: M. Ivanova, P. Stockhammer, B. Athanassov, V. Petrova, D. Takorova (Hrsg.), Social Dimensions of Food in the Prehistory of the Eastern Balkans and Neighbouring Areas. International Academy Conference, Heidelberg, 30 April - 2 May 2015, im Druck.

ReschreIter, KowARIK 2015

H. Reschreiter, K. Kowarik, Die prähistorischen Salzbergwerke von Hallstatt. In: Th. Stöllner, K. Oeggl (Hrsg.) Bergauf, Bergab: 10.000 Jahre Bergbau in den Ostalpen. Bochum 2015, 289-296.

RIEMER 1997

H. Riemer, Zur Form und Funktion: Zur systematischen Aufnahme und vergleichenden Analyse prähistorischer Gefäßkeramik, Archäologische Informationen 20, 1997, 117-131.

Russ-Popa 2008

G. Russ-Popa, Leder, Fell und Haut: Wichtige Rohstoffe für den prähistorischen Bergbau. In: A. Kern, K. Kowarik, A. W. RausCh, H. Reschreiter (Hrsg.), Salz-Reich: 7000 Jahre Hallstatt. Veröffentlichungen der Prähistorischen Abteilung 2, Wien 2008, 102-105. 
Stefanovich, Bankoff 1998

M. Stefanovich, A. Bankoff, Kamenska Čuka 1993-1995. In: M. Stefanovich, H. Todorova, H. Hauptmann (Hrsg.), James Harvey Gaul in Memoriam. In the Steps of James Harvey Gaul 1, Sofia 1998.

StÖllner, Oeggl 2015

Th. Stöllner, K. Oeggl (Hrsg.) Bergauf, Bergab: 10.000 Jahre Bergbau in den Ostalpen. Bochum 2015.

VALLA 2000

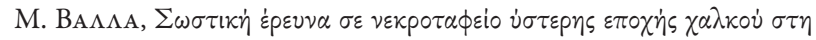

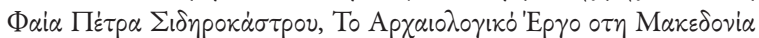

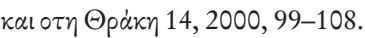

WardLE, WardLe 2007

K. A. WARDLE, D. WARDLE, Assiros Toumba: a brief history of the settlement. In: H. Todorova, M. Stefanovich, G. Ivanov (Hrsg.), The Struma/Strymon River Valley in Prehistory. Proceedings of the International Symposium „Strymon Praehistoricus“, Kjustendil-Blagoevgrad-Serres-Amphipolis, 27.09-01.10.2004. In The Steps of James Harvey Gaul 2, Sofia 2007, 451-479.

Barbara Horejs Institut für Orientalische und Europäische Archäologie Österreichische Akademie der Wissenschaften Hollandstraße 11-13 1020 Wien Österreich barbara.horejs@oeaw.ac.at iD orcid.org/0000-0002-4818-6268 